REPRESENTATION THEORY

An Electronic Journal of the American Mathematical Society

Volume 15, Pages 63-111 (January 24, 2011)

S $1088-4165(2011) 00377-0$

\title{
ELLIPTIC CENTRALIZERS IN WEYL GROUPS AND THEIR COINVARIANT REPRESENTATIONS
}

\author{
MARK REEDER
}

\begin{abstract}
The centralizer $C(w)$ of an elliptic element $w$ in a Weyl group has a natural symplectic representation on the group of $w$-coinvariants in the root lattice. We give the basic properties of this representation, along with applications to $p$-adic groups - classifying maximal tori and computing inducing data in $L$-packets - as well as to elucidating the structure of the centralizer $C(w)$ itself. We give the structure of each elliptic centralizer in $W\left(E_{8}\right)$ in terms of its coinvariant representation, and we refine Springer's theory for elliptic regular elements to give explicit complex reflections generating $C(w)$. The case where $w$ has order three is examined in detail, with connections to mathematics of the nineteenth century. A variation of the methods recovers the subgroup $W\left(H_{4}\right) \subset W\left(E_{8}\right)$.
\end{abstract}

\section{INTRODUCTION}

Let $W=W(R)$ be the Weyl group of an irreducible root system $R$ with root lattice $X=\mathbb{Z} R$. For each $w \in W$, the coinvariants $X_{w}:=X /(1-w) X$ form a finitely generated abelian group with an action of the centralizer $C(w)$ of $w$ in $W$. We say that $w$ is elliptic if $X_{w}$ is finite. In this case $X_{w}$ is a finite abelian group of order equal to the determinant of $1-w$ on $X$. Using the minimal polynomial $M(t)$ of $w$, one can define a canonical alternating bilinear form $\langle\cdot, \cdot\rangle_{w}$ on $X_{w}$, taking values in $\mathbb{Z} / m \mathbb{Z}$, where $m=M(1)$. This form is preserved by the action of $C(w)$, so we have a homomorphism

$$
\varrho_{w}: C(w) \longrightarrow S p\left(X_{w}\right),
$$

called the coinvariant representation of $C(w)$, where $S p\left(X_{w}\right)$ is the isometry group of the form $\langle\cdot, \cdot\rangle_{w}$. More generally, the normalizer $N(w)$ of the cyclic group generated by $w$ acts on $X_{w}$, now by similitudes of $\langle\cdot, \cdot\rangle_{w}$, and $\varrho_{w}$ is the restriction of the representation

$$
\tilde{\varrho}_{w}: N(w) \longrightarrow G S p\left(X_{w}\right) \text {. }
$$

These and other basic facts about coinvariant representations are proved in section 1 below.

This study of coinvariant representations was motivated by various problems in the structure and representation theory of reductive groups over $p$-adic fields, arising in recent work on the local Langlands correspondence ([13, [17, 21], 30]; see [16] for an introduction). These problems are of two related types: conjugacy of maximal tori and the structure of certain supercuspidal $L$-packets. Preliminary

Received by the editors June 9, 2009 and, in revised form, February 3, 2010.

2010 Mathematics Subject Classification. Primary 11E72, 20G05, 20G25.

(C)2011 American Mathematical Society 
to this is the basic question of the structure of $C(w)$ itself, for which we have no general theory. We now give a brief description of these problems and how coinvariant representations help to solve them.

Conjugacy of maximal tori: In a connected reductive group $G$ over an algebraically closed field, all maximal tori are conjugate. If $G$ is defined over a general field $k$, then the maximal tori in $G$ which are defined over $k$ are in general not conjugate to one another by the group $G(k)$ of $k$-rational points in $G$. Assume that $k$ is perfect with absolute Galois group $\Gamma$ and that $G$ is quasi-split over $k$ with maximal torus $T$ contained in a $k$-rational Borel subgroup of $G$. Let $W$ be the absolute Weyl group of $T$. The classification of rational conjugacy classes of maximal tori in $G$ (and in inner forms of $G$ ) can be approached in two steps, analogously to the theory of $L$-packets. First, one partitions the tori into "stable" classes $\mathcal{T}_{\varphi}$, indexed by cohomology classes of Galois cocycles $\varphi: \Gamma \rightarrow W$. Second, the rational classes in $\mathcal{T}_{\varphi}$ are in bijection with the orbits in the cohomology $H^{1}\left(k, T_{\varphi}\right)$ of the twisted torus $T_{\varphi}$ under a canonical affine action of the group $W_{\varphi}(k)$ of $k$-rational points in the twist of $W$ by $\varphi$. This follows from standard Galois cohomology arguments and a result of Raghunathan; see Proposition 6.5 below.

When $k$ is $p$-adic, this affine action is closely related to coinvariant representations. For simplicity, assume here that $G$ is split over $k$ and simply connected, so that $X=X_{*}(T)=\mathbb{Z} R$, where $R$ is the coroot lattice of $T$. A Galois cocycle is now just a homomorphism $\varphi: \Gamma \rightarrow W$, which we regard as a Langlands parameter for the stable class $\mathcal{T}_{\varphi}$. The tori in $\mathcal{T}_{\varphi}$ are anisotropic exactly when the coinvariant space $X_{\varphi}$ is finite, in which case Tate-Nakayama duality gives an isomorphism $H^{1}\left(k, T_{\varphi}\right) \simeq X_{\varphi}$. The classification of anisotropic tori in $G$ may be summarized as follows.

Theorem 1.1 (See Thm. 6.9). The $W$-conjugacy classes of Langlands parameters $\varphi: \Gamma \rightarrow W$ with $X_{\varphi}$ finite are in bijection with the stable classes $\mathcal{T}_{\varphi}$ of anisotropic maximal tori in $G$. Further, we have

1. $W_{\varphi}(k)$ is isomorphic to the centralizer $C(\varphi)$ of $\varphi$ in $W$ and $H^{1}\left(k, T_{\varphi}\right) \simeq$ $X_{\varphi}$.

2. There is a canonical cohomology class $\Delta_{\varphi} \in H^{1}\left(C(\varphi), X_{\varphi}\right)$ such that the rational classes in $\mathcal{T}_{\varphi}$ are in bijection with the orbits of $C(\varphi)$ in $X_{\varphi}$ under the affine action obtained by twisting the coinvariant representation by a cocycle belonging to the class of $\Delta_{\varphi}$.

Thus, the classification of anisotropic maximal tori is reduced to two problems: Find the orbits of $C(\varphi)$ on $X_{\varphi}$, and compute the class $\Delta_{\varphi}$. If $\varphi(\Gamma)$ is cyclic, generated by an elliptic element $w \in W$, then $X_{\varphi}=X_{w}$ is the coinvariant representation of $C(w)$ studied above. In Proposition 6.10 we show that the class $\Delta_{\varphi}$ is trivial if $\varphi$ is unramified, recovering a result from [13] with a more conceptual proof. In Proposition 6.18 we compute $\Delta_{\varphi}$ when $\varphi$ is totally and tamely ramified and $w$ is elliptic regular.

If $\varphi$ is only assumed to be tamely ramified with inertial image generated by an elliptic element $w \in W$, then the image $u$ of Frobenius lies in the normalizer $N(w)$. (Parameters of this type arise from the simple wild Langlands parameters of [17.) Now $W_{\varphi}(k)=C(w)^{u}$ is the centralizer of $u$ in $C(w)$ and $H^{1}\left(k, T_{\varphi}\right)=\left(X_{w}\right)_{u}$ is the space of coinvariants of the similitude $u$ acting on $X_{w}$ under the coinvariant representation of $N(w)$. Here we have less information about $\Delta_{\varphi}$, but can show that it vanishes whenever $\operatorname{det}(1-w)$ is odd. 
We also note that the invariants $\left(X_{w}\right)^{u}$ are isomorphic to the component group scheme of the lft Neron model of $T_{\varphi}$ (cf. [24, 7.6] and [28]). This group scheme appears in the construction of supercuspidal representations of $G(k)$ [18.

Supercuspidal L-packets: Given a $p$-adic group $G$ as above, with elliptic $w \in W$, unramified twist $T_{w}$ and a sufficiently nice character $\chi: T_{w}(k) \rightarrow \mathbb{C}^{\times}$, one can construct (cf. [13] 21] and [30]) a finite set $\Pi_{w}(\chi)$ of supercuspidal representations of $G(k)$, each induced from a certain maximal compact subgroup of $G(k)$. Since $G(k)$ has several conjugacy classes of maximal compact subgroups, we wish to determine which ones arise for each representation in $\Pi_{w}(\chi)$.

The set $\Pi_{w}(\chi)$ constitutes an $L$-packet in accordance with the local Langlands conjecture. Among other things, this means we have a bijection $X_{w} \rightarrow \Pi_{w}(\chi)$, denoted $\rho \mapsto \pi_{w}(\chi, \rho)$, with the property that $\pi_{w}(\chi, \rho)$ and $\pi_{w}\left(\chi, \rho^{\prime}\right)$ are induced from the same maximal compact subgroup if $\rho$ and $\rho^{\prime}$ belong to the same orbit of $C(w)$ in $X_{w}$. Moreover, the point stabilizers of $C(w)$ in $X_{w}$ determine the maximal compact subgroups appearing in $\Pi_{w}(\chi)$. Thus, the coinvariant representation can be used to determine the inducing data of the representations in $\Pi_{w}(\chi)$. See section 6.8. For $G$ of type $E_{8}$, one can read off the inducing maximal compact subgroups in the $L$-packets $\Pi_{w}(\chi)$ from Table 1 of section 2.3

Centralizers in Weyl groups: In the study of root systems, it is a basic question to ask for the structure of the centralizers of elements in a Weyl group $W$. The conjugacy classes in $W$ were determined by Carter [6] using the root system. He also found the orders of the centralizers, but not their group structure.

If $W$ is of classical type, it is straightforward to describe all centralizers using the presentation of $W$ as permutations and sign changes. Among exceptional Weyl groups, the most interesting is $W=W\left(E_{8}\right)$, our main focus from now on.

The coinvariant representation can be useful for describing the structure of $C(w)$, as in the following well-known example (cf. [3], 29]): Let $W=W\left(E_{8}\right)$ with $w=-1$. Then $C(w)=W$ and $X_{w}=X / 2 X$, on which $W$ preserves the quadratic form $q(x)=\frac{1}{2}\langle x, x\rangle \bmod 2$, where $\langle$,$\rangle is the symmetric W$-invariant pairing on $X$ such that roots have squared length two. The image of $\varrho_{w}$ turns out to be the full orthogonal group of $q$, so $W$ is presented as a covering:

$$
1 \longrightarrow\langle-1\rangle \longrightarrow W \longrightarrow O_{8}^{+}(2) \longrightarrow 1 \text {. }
$$

For any elliptic $w \in W\left(E_{8}\right)$, the form $\langle,\rangle_{w}$ is nondegenerate and satisfies $\langle x, x\rangle_{w}=$ 0 for all $x \in X_{w}$. The coinvariant representation often gives a similar description of $C(w)$ as a covering of a classical group. To illustrate, using Carter's notation for conjugacy classes: for $w=A_{2}^{4}$ we have the exact sequence

$$
1 \longrightarrow\langle w\rangle \longrightarrow C(w) \stackrel{\varrho_{w}}{\longrightarrow} S p_{4}(3) \longrightarrow 1,
$$

and for $w=A_{1}^{4} D_{4}$, we have the exact sequence

$$
1 \longrightarrow\langle w\rangle \longrightarrow C(w) \stackrel{\varrho_{w}}{\longrightarrow} O_{6}^{-}(2) \longrightarrow 1 .
$$

Often $\varrho_{w}$ is surjective (as in (4)) or has a relatively large image (as in (5)). Table 1 in section 2.3 gives the description of all elliptic centralizers $C(w)$ in $W\left(E_{8}\right)$ in terms of their coinvariant representations $\varrho_{w}$.

Regular elements, complex reflection groups, graded Lie algebras and cyclotomic structures: Following Springer, an element $w \in W$ is called regular if some eigenvector $v$ of $w$ in $\mathbb{C} \otimes X$ has trivial stabilizer in $W$. Springer showed that when $w$ is regular, the centralizer $C(w)$ acts faithfully as a complex reflection group on the 
$w$-eigenspace containing $v$, and that the degrees of the complex reflection group $C(w)$ are those degrees of $W$ which are divisible by the order of $w$. Knowing the degrees, one can usually locate $C(w)$ in the Shephard-Todd classification of complex reflection groups. However, Springer uses invariant theory to characterize reflection groups, a method which does not produce the actual reflections in $C(w)$ in an obvious way. Therefore it remains to complete Springer's theory of regular elements by finding the complex reflections which generate $C(w)$, for regular $w$. In section 3 we do this for those $w$ with irreducible minimal polynomial on $X$; we call such elements cyclotomic.

When $w$ is cyclotomic we find the reflections generating $C(w)$ by viewing $V:=$ $\mathbb{Q} \otimes X$ as a vector space over a the cyclotomic field $K=\mathbb{Q}(w) \subset \operatorname{End}(V)$. See Proposition 3.8. The reflections arise from an equivalence relation on the set of roots, via the action of $K$. Each equivalence class is a root subsystem of $S \subset R$ which determines a cyclic subgroup of $C(w)$ generated by a complex reflection. The possibilities for $S$ are classified, and these reflections are shown to generate $C(w)$ in section 3.2 .

It is a remarkable fact that in the Weyl groups of types $G_{2}, F_{4}$ and $E_{8}$, the cyclotomic elements are precisely those which are both elliptic and regular. Hence the centralizer of an elliptic regular element $w \in W\left(E_{8}\right)$ of order $d$ is the automorphism group of a cyclotomic $\mathbb{Z}\left[\zeta_{d}\right]$-structure on the $E_{8}$-root lattice $X\left(E_{8}\right)$, where $\zeta_{d}$ is a complex root of unity of order $d$. Some of these cyclotomic structures, e.g., for $d=3,4$, were found by ad hoc methods in the nineteenth century [8]. Recent literature on lattice theory [2] mentions the $\mathbb{Z}\left[\zeta_{15}\right]$-structure on $X\left(E_{8}\right)$. Indeed, all of the cyclotomic structures on $X\left(E_{8}\right)$ are unified by viewing them as arising from elliptic regular elements in $W\left(E_{8}\right)$. Section 6 describes the equivalence classes $S$ and hence the structure of the reflection group $C(w)$ for each cyclotomic class in $W\left(E_{8}\right)$.

Cyclotomic elements also appear in Vinberg's theory of graded Lie algebras [39. If $\mathfrak{g}$ is a simple complex Lie algebra and $G_{\sigma}$ is the identity component of the centralizer of an automorphism $\sigma \in \operatorname{Aut}(\mathfrak{g})$ of order $d$, then the representation of $G_{\sigma}$ on the $\zeta_{d}$-eigenspace $\mathfrak{g}_{\sigma}$ of $\sigma$ shares many properties with the adjoint representation of $G$ on $\mathfrak{g}$. In particular, the closed orbits of $G_{\sigma}$ in $\mathfrak{g}_{\sigma}$ are controlled by a finite group $W_{\sigma}$ analogous to $W$. Vinberg [39, Prop. 19] shows that if $\sigma$ is an inner automorphism normalizing a Cartan subalgebra and acting there via a cyclotomic element $w \in W$, then $W_{\sigma} \simeq C(w)$ and $W_{\sigma}$ is, in particular, a complex reflection group. Thus, our results give the reflections generating $W_{\sigma}$ in this case.

Elliptic Trialities: When $w$ is cyclotomic, the coinvariant group $X_{w}$ is zero unless the order $d$ of $w$ is a power of a prime $p$, in which case $X_{w}$ is the reduction modulo $\mathfrak{P}$ of the $\mathbb{Z}\left[\zeta_{d}\right]$-module $X$, where $\mathfrak{P}=\left(1-\zeta_{d}\right) \mathbb{Z}\left[\zeta_{d}\right]$ is the unique prime ideal of $\mathbb{Z}\left[\zeta_{d}\right]$ ramified over $\mathbb{Z}$. This interplay between complex and modular representations, while useful in both directions, is especially striking when $w$ is elliptic of order $p=3$. Here it is worthwhile to consider all root systems where this occurs, as each has unique features which are related to geometric structures investigated by Maschke and his contemporaries, and later by Coxeter. These are discussed in Appendix A

Finally, in Appendix B we use a variant of our method for producing reflections out of cyclotomic structures to explain the (known, cf. [26]) embedding of the 
noncrystallographic Coxeter group $W\left(H_{4}\right)$ in $W\left(E_{8}\right)$ as the centralizer of a $\mathbb{Z}[\tau]$ structure on the $E_{8}$ root lattice, where $\tau$ is the golden ratio.

\section{ThE COINVARIANT REPRESENTATION}

2.1. Quadratic lattices. Let $X$ be a $\mathbb{Z}$-lattice in a $\mathbb{Q}$-vector space $V$ of dimension $n$, and suppose we have a positive definite quadratic form $\langle$,$\rangle on V$ taking integer values on $X$. Let $A=A(X)$ be the (finite) subgroup of the orthogonal group $O(V)$ preserving $X$. We assume that $w \in A$ is elliptic, that is, $w$ has no nonzero fixed vectors in $V$. Equivalently, the group of coinvariants

$$
X_{w}:=X /(1-w) X
$$

is finite, of order $\left|X_{w}\right|=\operatorname{det}(1-w)=F(1)$, where $F(t)=\operatorname{det}(t-w)$ is the characteristic polynomial of $w$ in $\operatorname{End}(X)$. Since $w$ is of finite order and preserves $X$, its characteristic polynomial factors as a product of cyclotomic polynomials

$$
F(t)=\Phi_{d_{1}}(t)^{e_{1}} \cdot \Phi_{d_{2}}(t)^{e_{2}} \cdots \Phi_{d_{k}}(t)^{e_{k}},
$$

where $d_{1}<d_{2}<\cdots<d_{k}$ and the $e_{i}$ are positive integers. Here $\Phi_{d}(t)$ is the minimal polynomial of the complex roots of unity of order $d$. Since $w$ is elliptic we have $d_{1} \geq 2$, and

$$
F(1)=\prod_{i \in I} \ell_{i}^{e_{i}},
$$

where $I$ is the set of indices $i \in\{1,2, \ldots, k\}$ for which $d_{i}$ is a power of a prime $\ell_{i}$. The minimal polynomial of $w$ in $\operatorname{End}(X)$ is

$$
M(t)=\Phi_{d_{1}}(t) \cdot \Phi_{d_{2}}(t) \cdots \Phi_{d_{k}}(t)
$$

and the integer

$$
m:=M(1)=\prod_{i \in I} \ell_{i}
$$

divides $F(1)$.

Lemma 2.1. The integer $m=M(1)$ divides the order $d$ of $w$.

Proof. We have $\Phi_{d}(1)=p$ if $d$ is a power of a prime $p$, and $\Phi_{d}(1)=1$ if $d$ is not a prime power. Hence a prime $p$ appears in $m$ with exponent $e_{p}$ equal to the number of $d_{i}$ which are powers of $p$. Since the $d_{i}$ are distinct, we have $e_{p} \leq f_{p}$, where $p^{f_{p}}$ is the largest power of $p$ among the $d_{i}$ 's. Since $d=\operatorname{gcd} d_{i}$ is divisible by each $p^{f_{p}}$, the result follows.

The polynomial

$$
\dot{M}(t)=\frac{M(t)-M(1)}{t-1}
$$

also has integer coefficients, and satisfies

$$
(1-t) \dot{M}(t)+M(t)=m
$$

Let $\mathbb{Z}[w]$ be the $\mathbb{Z}$-subalgebra of $\operatorname{End}(X)$ generated by $w$. In the ring $\mathbb{Z}[w]$, we have the equation

$$
(1-w) \dot{M}(w)=m
$$

It follows that $m X \subset(1-w) X$, so that $m X_{w}=0$ and $X_{w}$ is a $\mathbb{Z} / m \mathbb{Z}$-module. This also implies that we have a well-defined pairing

$$
\langle,\rangle_{w}: X_{w} \times X_{w} \rightarrow \mathbb{Z} / m \mathbb{Z}
$$


given by

$$
\langle x, y\rangle_{w}=\left\langle\lambda_{x}, \dot{M}(w) \lambda_{y}\right\rangle \bmod m
$$

where $x, y \in X_{w}$ have lifts $\lambda_{x}, \lambda_{y} \in X$.

Lemma 2.2. The pairing $\langle,\rangle_{w}$ is skew-symmetric: $\langle x, y\rangle_{w}=-\langle y, x\rangle_{w}$ for all $x, y \in X_{w}$. If the quadratic lattice $X$ is even, then $\langle x, x\rangle_{w}=0$ for all $x \in X_{w}$.

Proof. Since $w$ preserves the form $\langle$,$\rangle , we have$

$$
\langle\mu, \dot{M}(w) \lambda\rangle=\left\langle\lambda, \dot{M}\left(w^{-1}\right) \mu\right\rangle
$$

for all $\lambda, \mu \in X$. But $M(t)$ is also the minimal polynomial of $w^{-1}$, so

$$
\left(1-w^{-1}\right) \dot{M}\left(w^{-1}\right)=m=(1-w) \dot{M}(w)
$$

or

$$
-w^{-1}(1-w) \dot{M}\left(w^{-1}\right)=(1-w) \dot{M}(w) .
$$

Since $1-w$ is a unit in $\operatorname{End}(V)$, this implies that

$$
\dot{M}\left(w^{-1}\right)=-w \dot{M}(w)=(1-w) \dot{M}(w)-\dot{M}(w)=m-\dot{M}(w) .
$$

This implies the first assertion. Since $w$ preserves the symmetric form $\langle$, $\rangle$, we have, for all $\lambda \in X$,

$$
\langle\lambda, \dot{M}(w) \lambda\rangle=\left\langle\lambda, \dot{M}\left(w^{-1}\right) \lambda\right\rangle,
$$

so (7) also implies that

$$
\langle\lambda, \dot{M}(w) \lambda\rangle=\langle\lambda, m-\dot{M}(w) \lambda\rangle
$$

or

$$
\langle\lambda, \dot{M}(w) \lambda\rangle=m \cdot \frac{\langle\lambda, \lambda\rangle}{2} \in m \mathbb{Z}
$$

if $\langle\lambda, \lambda\rangle \in 2 \mathbb{Z}$. This proves the last assertion.

We say the form $\langle,\rangle_{w}$ is nondegenerate if the radical

$$
X_{w}^{0}:=\left\{u \in X_{w}:\left\langle u, X_{w}\right\rangle_{w}=0\right\}
$$

is zero. We can determine $X_{w}^{0}$ using the dual lattice

$$
\hat{X}:=\{\lambda \in V:\langle\lambda, X\rangle \subset \mathbb{Z}\} .
$$

Note that $X \subseteq \hat{X}$ because we have assumed that $\langle$,$\rangle is integer-valued on X$.

Lemma 2.3. We have

$$
X_{w}^{0}=\frac{X \cap(1-w) \hat{X}}{(1-w) X}=\operatorname{ker}\left[X_{w} \rightarrow \hat{X}_{w}\right],
$$

where the latter map is induced by the inclusion $X \hookrightarrow \hat{X}$. If $X=\hat{X}$ is self-dual, then the form $\langle,\rangle_{w}$ is nondegenerate for every elliptic $w \in A(X)$.

Proof. Let $\lambda \in X$ have image $x \in X_{w}$. By (6) we have

$$
\begin{aligned}
\left\langle x, X_{w}\right\rangle_{w}=0 & \Leftrightarrow\langle\dot{M}(w) \lambda, X\rangle \subset m \mathbb{Z} \\
& \Leftrightarrow \dot{M}(w) \lambda \in m \hat{X} \\
& \Leftrightarrow \dot{M}(w) \lambda \in \dot{M}(w)(1-w) \hat{X} \\
& \Leftrightarrow \lambda \in(1-w) \hat{X},
\end{aligned}
$$

since $\dot{M}(w)$ is invertible on $V$. The lemma follows. 
Let $C_{A}(w)$ be the centralizer of $w$ in $A(X)$. This group acts naturally on $X_{w}$, giving a canonical homomorphism

$$
\varrho_{w}: C_{A}(w) \rightarrow S p\left(X_{w}\right),
$$

where $S p\left(X_{w}\right)$ is the group of automorphisms of the group $X_{w}$ preserving the form $\langle,\rangle_{w}$. We call $\varrho_{w}$ the coinvariant representation of $C_{A}(w)$. Clearly $\varrho_{w}$ contains $w$ in its kernel. Often, $\operatorname{ker} \varrho_{w}$ is generated by $w$, but this is not always the case, and the following Lemma can be used to determine ker $\varrho_{w}$ in particular cases.

Lemma 2.4. An element $v \in C_{A}(w)$ belongs to ker $\varrho_{w}$ exactly when the matrix of $(I-v) \cdot(I-w)^{-1}$ with respect to a basis of $X$ is integral.

Proof. This is immediate from the observation that $\varrho_{w}(v)=1$ iff $(1-v) X \subset$ $(1-w) X$.

The representation $\varrho_{w}$ extends to a representation $\tilde{\varrho}_{w}: N_{A}(w) \rightarrow \operatorname{Aut}\left(X_{w}\right)$ of the subgroup $N_{A}(w) \subset A(X)$ normalizing the group $\langle w\rangle$ generated by $w$. If $u \in N_{A}(w)$, then $u w u^{-1}=w^{s(u)}$, where $s(u)$ is an integer prime to the order $d$ of $w$. Since $m$ divides $d$, we obtain a homomorphism $\sigma: N_{A}(w) \rightarrow(\mathbb{Z} / m \mathbb{Z})^{\times}$, defined by $\sigma(u)=s(u)+m \mathbb{Z}$.

Proposition 2.5. For every $u \in N_{A}(w)$ and $x, y \in X_{w}$, we have

$$
\left\langle\tilde{\varrho}_{w}(u) x, \tilde{\varrho}_{w}(u) y\right\rangle_{w}=\sigma(u)\langle x, y\rangle_{w} .
$$

In particular, $\tilde{\varrho}_{w}$ sends $N_{A}(w)$ to the similitude group $G S p\left(X_{w}\right)$.

Proof. We must show that

$$
\langle u \lambda, \dot{M}(w) u \mu\rangle \equiv \sigma(u)\langle\lambda, \dot{M}(w) \mu\rangle \bmod m,
$$

or equivalently,

$$
\langle\lambda, \dot{M}(w) \mu\rangle \equiv \sigma(u)\left\langle u^{-1} \lambda, \dot{M}(w) u^{-1} \mu\right\rangle \quad \bmod m,
$$

for all $\lambda, \mu \in X$. Since $u$ preserves the form $\langle$,$\rangle on X$ and $u \dot{M}(w) u^{-1}=\dot{M}\left(w^{s(u)}\right)$, it suffices to show that

$$
\dot{M}(w) \equiv s \dot{M}\left(w^{s}\right) \quad \bmod m \mathbb{Z}[w],
$$

for every positive integer $s$ prime to $m$. For this, we may assume $s=p$ is a prime not dividing $m$. Viewing our problem in $\mathbb{Z}[t]$, we must show that $\dot{M}(t)-p \dot{M}\left(t^{p}\right)$ belongs to the ideal generated by $m$ and $M(t)$ in $\mathbb{Z}[t]$.

Since $M(t)=\Phi_{d_{1}}(t) \cdot \Phi_{d_{2}}(t) \cdots \Phi_{d_{k}}(t)$ and $p$ does not divide any $d_{i}$, we have $M\left(t^{p}\right)=M(t) \cdot N(t)$, where

$$
N(t)=\Phi_{p d_{1}}(t) \cdot \Phi_{p d_{2}}(t) \cdots \Phi_{p d_{k}}(t)
$$

and $N(1)=1$. Hence

$$
\begin{aligned}
\Phi_{p}(t) \cdot\left[\dot{M}(t)-p \dot{M}\left(t^{p}\right)\right] & =\Phi_{p}(t) \cdot \frac{M(t)-m}{t-1}-p \Phi_{p}(t) \cdot \frac{M(t) N(t)-m}{t^{p}-1} \\
& =\Phi_{p}(t) \cdot \frac{M(t)-m}{t-1}-p \cdot \frac{M(t) N(t)-m}{t-1} \\
& =\left[\frac{\Phi_{p}(t)-p N(t)}{t-1}\right] \cdot M(t)+\left[\frac{p-\Phi_{p}(t)}{t-1}\right] \cdot m .
\end{aligned}
$$


Since $\Phi_{p}(1)=p$ and $N(1)=1$, the terms in square brackets in the last line of (9) belong to $\mathbb{Z}[t]$. It now suffices to show that $\Phi_{p}(t)$ is a unit in the ring $\mathbb{Z}[t] /(m, M(t))$. By the Chinese remainder theorem, we may assume $m$ is a power of a prime $\ell \neq p$. An argument like that of Hensel's lemma reduces us to the case $m=\ell$. But $\Phi_{p}(t)$ is a unit in $\mathbb{F}_{\ell}[t] /(M(t))$ since the roots of $\Phi_{p}$ and $M(t)$ in $\overline{\mathbb{F}}_{\ell}$ have relatively prime orders. This concludes the proof of Proposition 2.5.

2.2. Root systems. For the rest of the paper, $V$ is the $\mathbb{Q}$-vector space generated by an irreducible root system $R$. On $V$ we have a unique symmetric form $\langle$, which is invariant under the Weyl group $W=W(R)$ and is normalized so that

$$
1 \in\langle R, R\rangle \subset \mathbb{Z}
$$

In most cases, the normalization (10) makes $\langle\alpha, \alpha\rangle=2$ for each short root $\alpha \in R$. The exception is in type $B_{n}$, where $\langle\alpha, \alpha\rangle=1$ for each short root. For $X$ we can take any $W$-stable lattice in $V$ containing $\mathbb{Z} R$, on which the form $\langle$,$\rangle is integer-valued.$

2.3. Table of elliptic centralizers and coinvariants in $E_{8}$. Suppose $R$ has type $E_{8}$ and $X=\mathbb{Z} R$. Then $\langle\alpha, \alpha\rangle=2$ for all $\alpha \in R$, so that $\hat{X}=X$. The automorphism group $A(X)=W$ is the Weyl group of $R$ and we write $C(w)=C_{A}(w)$ for the centralizer in $W$ of an element $w \in W$. For any elliptic element $w \in W$, the form $\langle,\rangle_{w}$ is nondegenerate on $X_{w}$, by Lemma 2.3 . Hence $X_{w}$ and its character group are isomorphic as modules for $C(w)$. We can view $X$ and $W$ as the algebraic character group and Weyl group of a maximal torus $\hat{T}$ in a complex Lie group $\hat{G}$ of type $E_{8}$. The fixed-point subgroup $\hat{T}^{w}$ is canonically the character group of $X_{w}$, so the form $\langle,\rangle_{w}$ identifies $X_{w}=\hat{T}^{w}$ as $C(w)$-modules. Since $\hat{G}$ is simply-connected, the centralizer $\hat{G}_{t}$ is connected for every $t \in \hat{T}^{w}$ and the stabilizer $W_{t}$ of $t$ in $W$ is the Weyl group of $\hat{T}$ in $\hat{G}_{t}$. Since $w \in W_{t}$ is elliptic, it follows that $\hat{G}_{t}$ is semisimple, and $t$ belongs to one of nine conjugacy classes in $\hat{G}$, where the Dynkin diagram of $\hat{G}_{t}$ is obtained by removing a node from the extended Dynkin diagram of $\hat{G}$. In particular, the order of $t$ is at most six, for any elliptic $w \in W$.

In Table 1 we tabulate the conjugacy classes of elliptic elements $w \in W\left(E_{8}\right)$ in the notation of [6], along with their orders and characteristic polynomials. Classes which are negatives of each other have the same centralizer (but different coinvariants) and are grouped together. Next, we give the group structure of $X_{w}$, the order of $C(w)$ (obtained from [6]) and the kernel and image in the extension

$$
1 \longrightarrow \operatorname{ker} \varrho_{w} \longrightarrow C(w) \longrightarrow \operatorname{im} \varrho_{w} \longrightarrow 1
$$

Finally, we give the orbit decomposition of $X_{w}-\{0\}=\hat{T}^{w}-\{1\}$ under $C(w)$, where the orbit type of the orbit through $t \in \hat{T}^{w}$ is a type of the Dynkin diagram of the centralizer $\hat{G}_{t}$. The details behind these results and further information about each centralizer are given in later sections, indicated in the rightmost column in Table 1 . 
TABLE 1. Elliptic centralizers in $W\left(E_{8}\right)$ and their coinvariant representations

\begin{tabular}{|c|c|c|c|c|c|c|c|}
\hline Class of $w$ & $|w|$ & $\operatorname{det}(t-w)$ & $X_{w}$ & $|C(w)|$ & $\operatorname{ker} \varrho_{w} \bullet \operatorname{im} \varrho_{w}$ & nonzero orbit types & details \\
\hline$E_{8}$ & 30 & $\Phi_{30}$ & 0 & $2 \cdot 3 \cdot 5$ & $\langle w\rangle \bullet 1$ & - & - \\
\hline$E_{8}\left(a_{1}\right)$ & 24 & $\Phi_{24}$ & 0 & $2^{3} \cdot 3$ & $\langle w\rangle \bullet 1$ & - & - \\
\hline$E_{8}\left(a_{2}\right)$ & 20 & $\Phi_{20}$ & 0 & $2^{2} \cdot 5$ & $\langle w\rangle \bullet 1$ & - & - \\
\hline$E_{8}\left(a_{5}\right)$ & 15 & $\Phi_{15}$ & 0 & $2 \cdot 3 \cdot 5$ & $\left\langle E_{8}\right\rangle \bullet 1$ & - & - \\
\hline$E_{8}\left(a_{3}\right)$ & 12 & $\Phi_{12}^{2}$ & 0 & $2^{5} \cdot 3^{2}$ & {$\left[\left\langle w^{4}\right\rangle \times U_{2}(3)\right] \bullet 1$} & - & $\begin{array}{l}3.4 .5 \\
4.2 .4\end{array}$ \\
\hline$E_{8}\left(a_{6}\right)$ & 10 & $\Phi_{10}^{2}$ & 0 & $2^{3} \cdot 3 \cdot 5^{2}$ & {$\left[\langle-w\rangle \times S L_{2}(5)\right] \bullet 1$} & - & 3.4 .3 \\
\hline$A_{4}^{2}$ & 5 & $\Phi_{5}^{2}$ & $5^{2}$ & $2^{3} \cdot 3 \cdot 5^{2}$ & $\langle w\rangle \bullet S L_{2}(5)$ & $24\left[A_{4}^{2}\right]$ & 4.2 .1 \\
\hline$D_{8}\left(a_{3}\right)$ & 8 & $\Phi_{8}^{2}$ & $2^{2}$ & $2^{6} \cdot 3$ & {$\left[\langle w\rangle \cdot Q_{8}\right] \bullet S L_{2}(2)$} & $3\left[D_{8}\right]$ & $\begin{array}{l}3.4 .4 \\
4.2 .2 \\
\end{array}$ \\
\hline$E_{8}\left(a_{8}\right)$ & 6 & $\Phi_{6}^{4}$ & 0 & $2^{7} \cdot 3^{5} \cdot 5$ & {$\left[\langle-w\rangle \times S p_{4}(3)\right] \bullet 1$} & - & 3.4 .2 \\
\hline$A_{2}^{4}$ & 3 & $\Phi_{3}^{4}$ & $3^{4}$ & $2^{7} \cdot 3^{5} \cdot 5$ & $\langle w\rangle \bullet S p_{4}(3)$ & $80\left[A_{2} E_{6}\right]$ & $4.1 \mid \mathrm{A} .3$ \\
\hline$D_{4}\left(a_{1}\right)^{2}$ & 4 & $\Phi_{4}^{4}$ & $2^{4}$ & $2^{10} \cdot 3^{2} \cdot 5$ & {$\left[\langle w\rangle \cdot\left(2^{3} \cdot 2^{2}\right)\right] \bullet S p_{4}(2)$} & $15\left[D_{8}\right]$ & $\begin{array}{l}3.4 .1 \\
4.2 .3\end{array}$ \\
\hline$A_{1}^{8}$ & 2 & $\Phi_{2}^{8}$ & $2^{8}$ & $2^{14} \cdot 3^{5} \cdot 5^{2} \cdot 7$ & $\langle w\rangle \bullet O_{8}^{+}(2)$ & $120\left[A_{1} E_{7}\right]+135\left[D_{8}\right]$ & 1 \\
\hline$E_{8}\left(a_{4}\right)$ & 18 & $\Phi_{18} \Phi_{6}$ & 0 & $2 \cdot 3^{3}$ & {$[\langle w\rangle \times 3] \bullet 1$} & - & - \\
\hline$A_{8}$ & 9 & $\Phi_{9} \Phi_{3}$ & $3^{2}$ & $2 \cdot 3^{3}$ & $\langle w\rangle \bullet 6$ & $6\left[A_{8}\right]+2\left[A_{2} E_{6}\right]$ & 5.1 \\
\hline$E_{8}\left(a_{7}\right)$ & 12 & $\Phi_{12} \Phi_{6}^{2}$ & 0 & $2^{5} \cdot 3^{2}$ & {$\left[\langle w\rangle \times S L_{2}(3)\right] \bullet 1$} & - & \\
\hline$A_{2} E_{6}$ & 12 & $\Phi_{12} \Phi_{3}^{2}$ & $3^{2}$ & $2^{5} \cdot 3^{2}$ & $\langle w\rangle \bullet S L_{2}(3)$ & $8\left[E_{6} A_{2}\right]$ & 5.2 \\
\hline$A_{1}^{2} A_{3}^{2}$ & 4 & $\Phi_{4}^{2} \Phi_{2}^{4}$ & $4^{2} \cdot 2^{2}$ & $2^{11} \cdot 3^{2}$ & $\langle w\rangle \bullet S p\left(X_{w}\right)$ & $\begin{array}{c}48\left[A_{3} D_{5}\right]+12\left[A_{1} E_{7}\right] \\
+3\left[D_{8}\right]\end{array}$ & 5.10 \\
\hline$A_{5} A_{2} A_{1}$ & 6 & $\Phi_{6} \Phi_{3}^{2} \Phi_{2}^{2}$ & $3^{2} \cdot 2^{2}$ & $2^{5} \cdot 3^{3}$ & $\langle w\rangle \bullet\left[S L_{2}(3) \times S L_{2}(2)\right]$ & $\begin{array}{c}24\left[A_{5} A_{2} A_{1}\right] \\
+8\left[A_{2} E_{6}\right]+3\left[A_{1} E_{7}\right] \\
\end{array}$ & 5.2 \\
\hline$D_{8}$ & 14 & $\Phi_{14} \Phi_{2}^{2}$ & $2^{2}$ & $2^{2} \cdot 7$ & $\langle w\rangle \bullet 2$ & $1\left[A_{1} E_{7}\right]+2\left[D_{8}\right]$ & 5.1 \\
\hline$D_{8}\left(a_{2}\right)$ & 30 & $\Phi_{10} \Phi_{6} \Phi_{2}^{2}$ & $2^{2}$ & $2^{2} \cdot 3 \cdot 5$ & $\langle w\rangle \bullet 2$ & $1\left[A_{1} E_{7}\right]+2\left[D_{8}\right]$ & 5.1 \\
\hline$D_{8}\left(a_{1}\right)$ & 12 & $\Phi_{12} \Phi_{4}^{2}$ & $2^{2}$ & $2^{3} \cdot 3^{2}$ & $\langle w\rangle \bullet S L_{2}(2)$ & $3\left[D_{8}\right]$ & 2.4 \\
\hline$A_{7} A_{1}$ & 8 & $\Phi_{8} \Phi_{4} \Phi_{2}^{2}$ & $8 \cdot 2$ & $2^{7}$ & $\langle w\rangle \bullet \operatorname{Aut}\left(C_{8} \times C_{2}\right)$ & $\begin{array}{c}8\left[A_{7} A_{1}\right]+4\left[A_{3} D_{5}\right] \\
+2\left[A_{1} E_{7}\right]+1\left[D_{8}\right]\end{array}$ & 5.2 \\
\hline$A_{1}^{2} D_{6}$ & 10 & $\Phi_{10} \Phi_{2}^{4}$ & $2^{4}$ & $2^{4} \cdot 3 \cdot 5^{2}$ & $\langle w\rangle \bullet S_{5}$ & $10\left[A_{1} E_{7}\right]+5\left[D_{8}\right]$ & 5.6 \\
\hline$D_{4}^{2}$ & 6 & $\Phi_{6}^{2} \Phi_{2}^{4}$ & $2^{4}$ & $2^{5} \cdot 3^{4}$ & {$\left[\langle w\rangle \times S_{3}\right] \bullet\left[\left(S_{3}^{2}\right) \cdot 2\right]$} & $6\left[A_{1} E_{7}\right]+9\left[D_{8}\right]$ & 5.7 \\
\hline$A_{1}^{4} D_{4}$ & 6 & $\Phi_{6} \Phi_{2}^{6}$ & $2^{6}$ & $2^{8} \cdot 3^{5} \cdot 5$ & $\langle w\rangle \bullet O_{6}^{-}(2)$ & $36\left[A_{1} E_{7}\right]+27\left[D_{8}\right]$ & 5.8 \\
\hline$A_{3} D_{5}\left(a_{1}\right)$ & 12 & $\Phi_{6} \Phi_{4}^{2} \Phi_{2}^{2}$ & $4^{2}$ & $2^{6} \cdot 3^{2}$ & $\langle w\rangle \bullet S L_{2}(\mathbb{Z} / 4)$ & $12\left[A_{3} D_{5}\right]+3\left[D_{8}\right]$ & 5.9 \\
\hline$A_{2} E_{6}\left(a_{2}\right)$ & 6 & $\Phi_{6}^{2} \Phi_{3}^{2}$ & $3^{2}$ & $2^{6} \cdot 3^{3}$ & {$\left[\left\langle w^{2}\right\rangle \times S L_{2}(3)\right] \bullet S L_{2}(3)$} & $8\left[A_{2} E_{6}\right]$ & 5.5 \\
\hline$A_{1} E_{7}$ & 18 & $\Phi_{18} \Phi_{2}^{2}$ & $2^{2}$ & $2^{2} \cdot 3^{3}$ & $\langle w\rangle \bullet S L_{2}(2)$ & $3\left[A_{1} E_{7}\right]$ & 5.2 \\
\hline$A_{1} E_{7}\left(a_{2}\right)$ & 12 & $\Phi_{12} \Phi_{6} \Phi_{2}^{2}$ & $2^{2}$ & $2^{4} \cdot 3^{2}$ & {$[2 \times\langle w\rangle] \bullet S L_{2}(2)$} & $3\left[A_{1} E_{7}\right]$ & 5.3 \\
\hline$A_{1} E_{7}\left(a_{4}\right)$ & 6 & $\Phi_{6}^{3} \Phi_{2}^{2}$ & $2^{2}$ & $2^{5} \cdot 3^{5}$ & {$\left[ \pm C_{E_{6}}(w)\right] \bullet S L_{2}(2)$} & $3\left[A_{1} E_{7}\right]$ & 5.4 \\
\hline
\end{tabular}




\section{Cyclotomic Automorphisms of Root Systems}

In Table 1 above, we have listed first those classes in $W$ with irreducible minimal polynomials. The centralizers of such classes can be understood in a uniform way that is analogous to the description of centralizers in classical groups over fields (see e.g. 36]).

Let $W$ be a Weyl group of an irreducible root system $R$, acting on the root lattice $X=\mathbb{Z} R$. Set $V=\mathbb{Q} \otimes V$ and $\bar{V}=\overline{\mathbb{Q}} \otimes V$, where $\overline{\mathbb{Q}}$ is the algebraic closure of $\mathbb{Q}$ in $\mathbb{C}$. We say $w \in W$ is cyclotomic if its minimal polynomial $M(t)$ on $V$ is irreducible over $\mathbb{Q}$. This means that $M(t)$ is the cyclotomic polynomial $\Phi_{d}(t)$, where $d$ is the order of $w$. Extending the $W$-invariant pairing $\langle$,$\rangle to \bar{V}$, we say $w \in W$ is regular (cf. [35]) if $w$ has an eigenvector in $\bar{V}$ not orthogonal to any root in $R$.

Lemma 3.1. Every nonidentity cyclotomic element $w \in W$ is elliptic and regular. For $R$ of type $G_{2}, F_{4}, E_{8}$ every elliptic regular element in $W$ is cyclotomic.

Proof. If $w \in W$ is cyclotomic, the Galois group $\Gamma=\operatorname{Gal}(\overline{\mathbb{Q}} / \mathbb{Q})$ is transitive on the eigenvalues of $w$. This Galois action extends to $\bar{V}$, acting trivially on $V$, so as to commute with the $W$-action. Thus, $\Gamma$ permutes the eigenspaces of $w$ transitively.

Each root $\alpha \in R$ may be viewed as a linear functional on $\bar{V}$, via the pairing $\langle$,$\rangle .$ In this guise, the map $\alpha: \bar{V} \rightarrow \overline{\mathbb{Q}}$ commutes with the $\Gamma$-action on $\bar{V}$ and $\overline{\mathbb{Q}}$. Hence if $\alpha$ vanishes on one eigenspace of $w$, it must vanish on all eigenspaces, so that $\alpha=0$, a contradiction. Therefore every root $\alpha \in R$ restricts to a nonzero functional on every $w$-eigenspace in $\bar{V}$. Since $R$ is finite, it follows that every $w$-eigenspace in $\bar{V}$ contains a regular vector. In particular, $w$ is regular. It is clear that $w$ is elliptic if $w \neq 1$.

If $R$ has type $G_{2}, F_{4}$ or $E_{8}$, Springer's list of regular elements [35, 5.4] shows that all elliptic regular elements are cyclotomic.

In this chapter, we will sharpen Springer's results by finding the reflections in $C(w)$ for cyclotomic elements $w$ and then we work out the details for cyclotomic centralizers in type $E_{8}$.

3.1. Cyclotomic elements and exponents. The cyclotomic classes in $W$ can be classified in terms of the exponents of $W$.

If $v \in W$ has irreducible characteristic polynomial $\Phi_{e}(t)$, then for each divisor $d \mid e$, the element $w=v^{e / d}$ is cyclotomic, with minimal polynomial $\Phi_{d}(t)$. In fact, a case-by-case check shows that all cyclotomic elements $w \neq \pm 1$ can be found in this way:

Lemma 3.2. If $w \in W$ is cyclotomic of order $d>2$, then $w=v^{e / d}$ where $v \in W$ has irreducible characteristic polynomial $\Phi_{e}(t)$ and $d$ divides $e$.

In turn, the elements with irreducible characteristic polynomial can be classified as follows.

Lemma 3.3. Let $e \geq 2$ be an integer. Then the following are equivalent:

1. There exists $v \in W$ with characteristic polynomial $\Phi_{e}(t)$.

2. The exponents $\left\{m_{1}, \ldots, m_{n}\right\}$ of $W$ represent the cosets in $(\mathbb{Z} / e \mathbb{Z})^{\times}$.

If these conditions hold, then $v$ is regular, unique up to conjugacy, and the centralizer $C(v)=\left\langle v^{\prime}\right\rangle$ is cyclic of order equal to the unique degree $d_{i}=m_{i}+1$ of $W$ which is divisible by e. Conditions 1 and 2 also hold with $(e, v)$ replaced $\left(d_{i}, v^{\prime}\right)$. 
Proof. Assuming condition 1, regularity was proved by Springer in [35, 4.11] and also follows from the proof of Lemma 3.3 above. Uniqueness now follows from 35 , 4.2], which also shows that the eigenvalues of $v$ are $\eta^{m_{i}}, i=1, \ldots, n$, where $\eta \in \overline{\mathbb{Q}}^{\times}$ has order $e$. But these eigenvalues are the roots of $\Phi_{e}(t)$, so $\left\{m_{1}, \ldots, m_{n}\right\}$ is a system of representatives for $(\mathbb{Z} / e \mathbb{Z})^{\times}$.

Now assume condition 2 holds. We may assume $n \geq 2$. Then $n=\phi(e)$ is even. Moreover, for any prime $p \mid e$, we have the constraints

$$
p \leq n+1, \quad p \nmid m_{i}, \quad 1 \leq i \leq n .
$$

For $R=A_{n}$, with exponents $\{1,2, \ldots, n\}$, the second constraint implies that $p \geq n+1$. Hence $n=p-1$ for some prime $p$, and $v$ is a Coxeter element, with characteristic polynomial $\Phi_{p}(t)$.

For $B_{n}, C_{n}$, constraints (11) imply that $n$ is a power of 2 and $v$ is a Coxeter element in $W$, with characteristic polynomial $t^{n}+1=\Phi_{2 n}(t)$.

Consider $R=D_{n}$. We have seen that $n$ is even. But then $n-1$ appears twice as an exponent; conditions 1 and 2 never hold.

For $G_{2}, F_{4}, E_{6}, E_{8}$, there are few primes satisfying the constraints (11) and few possibilities for $e$ such that $\phi(e)=n$. With the exception of $e=4$ for $G_{2}$ and $e=16$ for $E_{8}$, there is an element $v \in W$ of order $e$. These are tabulated below, in the notation of [6], for conjugacy classes in $W$.

\begin{tabular}{|c|c|c|c|}
\hline$R$ & exponents & $e$ & $v$ \\
\hline \hline$G_{2}$ & 1,5 & 3,6 & $A_{2}, G_{2}$ \\
\hline$F_{2}$ & $1,5,7,11$ & 8,12 & $B_{4}, F_{4}$ \\
\hline$E_{6}$ & $1,4,5,7,8,11$ & 9 & $E_{6}\left(a_{1}\right)$ \\
\hline$E_{8}$ & $1,7,11,13,17,19,23,29$ & $15,20,24,30$ & $E_{8}\left(a_{5}\right), E_{8}\left(a_{2}\right), E_{8}\left(a_{1}\right), E_{8}$ \\
\hline
\end{tabular}

For the cases in this table, we have $C(v)=\langle v\rangle$, except for class $A_{2}$ in $G_{2}$ and $E_{8}\left(a_{5}\right)$, which are each the square of a Coxeter element $v^{\prime}$, and $C(v)=\left\langle v^{\prime}\right\rangle$.

Proposition 3.4. If $w \in W$ is cyclotomic, then $C(w)$ is irreducible on every eigenspace of $w$ in $\bar{V}$.

Proof. We may assume $w \neq \pm 1$. Let $w=v^{e / d}$ as in Lemma 3.2. We will actually show that $C(w) \cap N(v)$ is irreducible on every eigenspace of $w$, where $N(v)$ denotes the normalizer in $W$ of the subgroup $\langle v\rangle$ generated by $v$. There is a homomorphism

$$
\sigma: N(v) \longrightarrow(\mathbb{Z} / e \mathbb{Z})^{\times}
$$

defined by $n^{-1} v n=v^{\sigma(n)}$, for $n \in N(v)$. It follows from [35, 4.7] that $\sigma$ is surjective, so we have an exact sequence

$$
1 \longrightarrow C(v) \longrightarrow N(v) \stackrel{\sigma}{\longrightarrow}(\mathbb{Z} / e \mathbb{Z})^{\times} \longrightarrow 1,
$$

by which the group $(\mathbb{Z} / e \mathbb{Z})^{\times}$permutes the eigenspaces of $v$ in $\bar{V}$. The following fact is used implicitly in 35 .

Lemma 3.5. If $v \in W$ is regular, then $(\mathbb{Z} / e \mathbb{Z})^{\times}$freely permutes the regular eigenspaces of $v$. 
Proof. Let $E \subset \bar{V}$ be an eigenspace for $v$ containing a regular vector, and suppose $n \in N(v)$ preserves $E$. Since $v$ is a scalar on $E$, the commutator $[n, v]$ fixes $E$ pointwise. Therefore $[n, v]$ fixes a regular vector, so $[n, v]=1$.

Returning to the proof of Proposition 3.4, we have $w \in W$ of order $d$ with eigenvalue $\zeta$ and $w=v^{e / d}$ where $v \in W$ has characteristic polynomial $\Phi_{e}(t)$ and $d \mid e$. We also have $\zeta=\eta^{e / d}$, where $\eta$ is an eigenvalue of $v$. Let $\Delta$ be the kernel of the natural map $(\mathbb{Z} / e \mathbb{Z})^{\times} \longrightarrow(\mathbb{Z} / d \mathbb{Z})^{\times}$. The sequence (12) restricts to another exact sequence

$$
1 \longrightarrow C(v) \longrightarrow N(v) \cap C(w) \stackrel{\sigma}{\longrightarrow} \Delta \longrightarrow 1 .
$$

Since $v$ is regular with eigenvalues of multiplicity one, Lemma 3.5 implies that the group $\Delta$ freely permutes the eigenlines of $v$ in $\bar{V}$. On the other hand, the eigenvalues of $v$ in $\bar{V}(w, \zeta)$ are $\eta^{i}$, where $i \in \Delta$. Hence $\operatorname{dim} \bar{V}(w, \zeta)=|\Delta|$, so $\Delta$ is transitive on the $v$-eigenlines in $\bar{V}(w, \zeta)$. This shows that $N(v) \cap C(w)$ is irreducible on $\bar{V}(w, \zeta)$.

3.2. An equivalence relation. Fix a cyclotomic element $w \in W$. The $\mathbb{Q}$-algebra $K=\mathbb{Q}(w) \subset \operatorname{End}(V)$ is a field. Let $V_{K}$ be the abelian group $V$ viewed as a vector space over $K$. Every $\alpha \in R$ spans a $K$-line $K \alpha=\{f(w) \alpha: f \in \mathbb{Q}[t]\}$ in $V_{K}$. We say that two roots $\alpha, \beta \in R$ are $K$-equivalent if $K \alpha=K \beta$. This is an equivalence relation on $R$. For each $K$-equivalence class $S \subset R$, the rational span $\mathbb{Q} S$ is contained in $K \alpha$ for any $\alpha \in S$. Conversely, since $w \alpha$ is $K$-equivalent to $\alpha$ for any $\alpha \in S$, we have $K S \subset K \alpha$. This shows that $\mathbb{Q} S=K \alpha$ for any root $\alpha \in S$. We also note that

$$
S=R \cap \mathbb{Q} S .
$$

Equation (14) implies that $S$ is a root subsystem in $R$ of rank equal to the degree of $K$ over $\mathbb{Q}$. Let $A(S)$ and $W(S)$ denote the automorphism and Weyl groups of $S$, respectively. Then $W(S)$, being generated by reflections from $S$, is a subgroup of $W=W(R)$. However, the group $A(S)$ need not be contained in $A(R)$. For each $K$-equivalence class $S \subset R$, the subgroup

$$
C_{S}(w):=W(S) \cap C(w)
$$

consists of the $K$-linear elements of $W(S)$. If $r \in C_{S}(w)$ and $\alpha \in S$, then $r \cdot \alpha=$ $\eta_{S}(r) \alpha$ for some scalar $\eta_{S}(\alpha) \in K^{\times}$which is independent of $\alpha$. This defines an injective homomorphism

$$
\eta_{S}: C_{S}(w) \longrightarrow K^{\times} .
$$

It follows that the group $C_{S}(w)$ is cyclic of order dividing the number of roots of unity in $K^{\times}$.

The orthogonal form $\langle$,$\rangle on V$ gives rise to a hermitian form $h(x, y)$ on $V_{K}$ characterized by the identity

$$
\langle a x, y\rangle=\operatorname{tr}(a h(x, y)), \quad \text { for all } a \in K,
$$

where $\operatorname{tr}: K \rightarrow \mathbb{Q}$ is the trace. If $L$ is a $K$-line in $V$, then the orthogonal complements of $L$ with respect to $\langle$,$\rangle and h$ coincide as $\mathbb{Q}$-subspaces of $V$. Let $U\left(V_{K}, h\right)$ be the unitary group of the form $h$ on $V_{K}$. The centralizer $C(w)$ consists of the elements in $W$ which act $K$-linearly on $V_{K}$ and we have

$$
C(w)=W \cap U\left(V_{K}, h\right) .
$$


A $K$-reflection on $V_{K}$ is an element $g \in U\left(V_{K}, h\right)$ of finite order whose fixed-point set is a $K$-hyperplane. A $K$-reflection has exactly one $K$-eigenvalue $\eta \neq 1$ and $\eta=\operatorname{det}\left(w \mid V_{K}\right)$ is a root of unity in $K^{\times}$.

Lemma 3.6. Any nontrivial element $r \in C_{S}(w)$ is a $K$-reflection with nontrivial $K$-eigenvalue $\eta=\eta_{S}(r)$, pointwise fixing the $K$-hyperplane orthogonal to $S$ with respect to $h$, and having the formula

$$
r(x)=x-(1-\eta) \frac{h(x, \alpha)}{h(\alpha, \alpha)} \alpha,
$$

for any $\alpha \in S$.

Proof. Since $r \in W(S)$, it pointwise fixes the $\langle$, $\rangle$-orthogonal complement of $\mathbb{Q} S$ which coincides with the $h$-orthogonal complement of the $K$-line $K S=\mathbb{Q} S$. Since $r \in U\left(V_{K}, h\right)$, it follows that $r$ is a $K$-reflection. We have seen that $\eta$ is a nontrivial eigenvalue of $r$ occurring in the $K$-line $K S$. Since the right side of (16) also pointwise fixes the orthogonal complement of $K S$ and acts by the scalar $\eta$ on $K S$, it must agree with $r(x)$.

Lemma 3.7. Every $K$-reflection $r \in C(w)$ is contained in $C_{S}(w)$ for a unique $K$-equivalence class $S \subset R$.

Proof. Let $L$ be the nontrivial $K$-eigenline of $r$. Then the fixed-point set of $r$ in $V_{K}$ is the orthogonal complement $L^{\prime}$ of $L$ with respect to $h$. As $\mathbb{Q}$-vector spaces, $L^{\prime}$ is also the $\langle$,$\rangle -orthogonal complement L$. The subgroup $W^{\prime}$ of $W$ fixing $L^{\prime}$ pointwise is generated by reflections about the roots orthogonal to $L^{\prime}$ [3, V.3, Prop. 2]. The set $S$ of these roots is nonempty, since $1 \neq r \in W^{\prime}$. Since $S \subset L$, it follows that $S$ is a $K$-equivalence class and $r \in C_{S}(w)$. Uniqueness follows from equation (14).

Proposition 3.8. If $w \in W$ is cyclotomic, then $C(w)$ is generated by the cyclic subgroups $C_{S}(w)$, with $S$ ranging over the $K$-equivalence classes in $R$.

Proof. The centralizer $C(w)$ preserves each eigenspace of $w$ in $\bar{V}$. Hence for every eigenvalue $\zeta$ of $w$ we have a representation

$$
\pi_{\zeta}: C(w) \longrightarrow G L(\bar{V}(w, \zeta))
$$

on the $\zeta$-eigenspace $\bar{V}(w, \zeta)$ of $w$ in $\bar{V}$. Since $\bar{V}(w, \zeta)$ contains a regular vector, the map $\pi_{\zeta}$ is injective. By Springer's results [35, 4.2, 6.4], the image of $\pi_{\zeta}$ is generated by reflections.

The eigenspace $\bar{V}(w, \zeta)$ is defined over $K$, hence every reflection in the image of $\pi_{\zeta}$ has its nontrivial eigenvalue in $K$. Embedding $K \subset \overline{\mathbb{Q}}$ via $w \rightarrow \zeta$, we have a $C(w)$-equivariant isomorphism

$$
\overline{\mathbb{Q}} \otimes_{K} V_{K} \stackrel{\sim}{\longrightarrow} \bar{V}(w, \zeta)
$$

sending $v \in V_{K}$ to $\sum_{k=1}^{d} \zeta^{-k} w^{k} v$. Hence $\pi_{\zeta}$ maps the $K$-reflections in $C(w)$ bijectively onto the reflections in $\pi_{\zeta}(C(w))$. The result follows.

3.3. The possible equivalence classes. Next, we tabulate the possibilities for a $K$-equivalence class in $S \subset R$, where $K \subset \operatorname{End}(V)$ is the field generated over $\mathbb{Q}$ by a nontrivial cyclotomic element $w \in W$. This will allow us to verify that $C_{S}(w)$ is nontrivial. Let $d>1$ be the order of $w$.

By the definition of $K$-equivalence, we have $w S=S$. Thus, $w$ acts on the root system $S$ via an automorphism $w_{S} \in A(S)$ having characteristic polynomial 
TABle 2. Possible root systems $S_{1}$ and automorphisms $w_{1} \in A\left(S_{1}\right)$

\begin{tabular}{|c|c|c|c|}
\hline$S_{1}$ & $w_{1}$ & $e$ & $\ell$ \\
\hline \hline$A_{1}$ & $A_{1}$ & 2 & 1 \\
\hline$A_{p-1}$ & $A_{p-1},-A_{p-1}$ & $p, 2 p$ & 1,2 \\
\hline$B_{2^{r}}, C_{2^{r}}$ & $B_{2^{r}}$ & $2^{r+1}$ & 1 \\
\hline$D_{2^{r}}, r \geq 2$ & $B_{2^{r}}$ & $2^{r+1}$ & 2 \\
\hline$D_{4}$ & $F_{4}$ & 12 & 3 \\
\hline$E_{6}$ & $E_{6}\left(a_{1}\right),-E_{6}\left(a_{1}\right)$ & 9,18 & 1,2 \\
\hline$E_{8}$ & $E_{8}, E_{8}\left(a_{1}\right), E_{8}\left(a_{2}\right), E_{8}\left(a_{5}\right)$ & $30,24,20,15$ & 1 \\
\hline$F_{4}$ & $F_{4}, B_{4}$ & 12,8 & 1 \\
\hline$G_{2}$ & $G_{2}, A_{2}$ & 6,3 & 1 \\
\hline
\end{tabular}

$\Phi_{d}(t)$ on $\mathbb{Q} S$. This implies that the group generated by $w_{S}$ acts transitively on the irreducible components $S_{1}, \ldots, S_{c}$ of $S$, that $c \mid d$, and that $w_{S}^{c}=\left(w_{1}, \ldots, w_{c}\right)$, for certain elements $w_{i} \in A\left(S_{i}\right)$.

The possibilities for $S_{1}$ are given in Table 2 , using the notation of [6] for conjugacy classes in Weyl groups, extended to $A(R)$ in the obvious way. We set $e:=d / c$; this is the order of $w_{1}$ in $A\left(S_{1}\right)$. In the last column we give the order $\ell$ of $w_{1}$ in the quotient group $A\left(S_{1}\right) / W\left(S_{1}\right)$. In the second row $p$ is a prime $\geq 3$.

To arrive at Table 2 we first observe that on the $\mathbb{Q}$ vector space $\mathbb{Q} S$, the element $w_{S}^{c}$ has characteristic polynomial

$$
\operatorname{det}\left(t I-w_{S}^{c}\right)=\Phi_{e}(t)^{\phi(d) / \phi(e)}=\prod_{i=1}^{c} \operatorname{det}\left(t I-w_{i}\right),
$$

where $\operatorname{det}\left(t I-w_{i}\right)$ is the characteristic polynomial of $w_{i}$ on $\mathbb{Q} S_{i}$. By the transitivity of $w_{S}$ on the $S_{i}$, there is an integer $m \geq 1$ such that

$$
\operatorname{det}\left(t I-w_{i}\right)=\Phi_{e}(t)^{m}
$$

for all $i$. Comparing degrees in (17), we find that

$$
\phi(c e)=\phi(d)=m \cdot c \cdot \phi(e) .
$$

But equation (18) can only hold if $m=1$ and every prime dividing $d$ also divides $e$. It follows that $S_{1}$ is an irreducible root system of rank $\phi(e)$ admitting an automorphism $w_{1}$ with characteristic polynomial $\operatorname{det}\left(t I-w_{1}\right)=\Phi_{e}(t)$, such that $\phi(e) \mid \phi(d)$. These constraints lead to Table 2 .

Corollary 3.9. For each $K$-equivalence class $S \subset R$, the group $C_{S}(w)$ is nontrivial.

Proof. Recall that $w_{S} \in A(S)$ is the automorphism of $S$ induced by $w$. If $w_{S}^{\nu} \in$ $W(S)$ for some $\nu \geq 1$, then $w_{S}^{\nu}$ acts trivially on the orthogonal complement of $\mathbb{Q} S$ and acts on $\mathbb{Q} S$ as $w^{\nu}$. Hence $w_{S}^{\nu}$ commutes with $w$ on $V$, so that $w_{S}^{\nu} \in C_{S}(w)$.

Therefore it suffices to show that $w_{S}^{c \ell} \neq 1$. If $w_{S}^{c \ell}=1$, then $w_{1}^{\ell}=1$, which implies $e \mid \ell$. But according to Table 2, this does not happen. 
Corollary 3.10. The subgroup of $C(w)$ generated by the subgroups $C_{S}(w)$ as $S$ ranges over the $K$-equivalence classes containing a short root of $R$ acts irreducibly on $V_{K}$.

Proof. From the transitivity of $W$ on roots of a given length [3, VI.1, Prop. 11], it follows that for any two short roots $\alpha, \beta \in R$, there is a sequence

$$
\alpha=\alpha_{0}, \alpha_{1}, \ldots, \alpha_{k}=\beta
$$

of short roots in $R$ such that $\left\langle\alpha_{i}, \alpha_{i+1}\right\rangle \neq 0$ for $0 \leq i<k$.

The hermitian form $h(x, y)$ satisfies $T(h(x, y))=\langle x, y\rangle$. It follows that the sequence (19) also satisfies $h\left(\alpha_{i}, \alpha_{i+1}\right) \neq 0$ for $0 \leq i<k$.

Now suppose $U \subset V_{K}$ is a nonzero $K$-subspace preserved for all the groups $C_{S}(w)$ where $S$ contains a short root. Take a nonzero element $x \in U$. Since the short roots span $V$, there is $\alpha \in R$ such that $\langle x, \alpha\rangle \neq 0$. Let $S$ be the $K$-equivalence class containing $\alpha$. By Corollary 3.9, there is a nontrivial $K$-reflection $r_{S} \in C_{S}(w)$, given by the formula

$$
r_{S}(x)=x-(1-\eta) \frac{h(x, \alpha)}{h(\alpha, \alpha)} \alpha,
$$

where $1 \neq \eta \in \overline{\mathbb{Q}}^{\times}$. Since $T(h(x, \alpha))=\langle x, \alpha\rangle \neq 0$, this shows that $\alpha \in U$. Let $\beta \in R$ be an arbitrary short root, and choose a sequence as in (19). Repeating the previous argument with $x, \alpha$ replaced by $\alpha, \alpha_{1}$ shows that $\alpha_{1} \in U$. In this way, we see that $\beta \in U$. Hence all short roots are contained in $U$, so $U=V$.

One more consequence of Table 2 will be useful for our study of cyclotomic classes in $E_{8}$.

Corollary 3.11. Suppose $w \in A(R)$ is cyclotomic of even square-free order $d$. Then one of the following holds:

1. $w=-1$;

2. $R=G_{2}$ or $E_{8}$ and $w$ is a Coxeter element;

3. $d=2 p$, where $p \in\{3,5\}$. Each $K$-equivalence class $S$ has type $A_{p-1}, C_{S}(w)$ is generated by a Coxeter element in $W(S)$ and there are $|R| p^{-1}$ reflections in $C(w)$, each of order $p$.

Proof. Since $d$ is square-free and $e$ contains every prime divisor of $d$, we must have $e=d$, so $c=1$ and each $S=S_{1}$ is irreducible. The third column of Table 2 gives the asserted possibilities for $S$.

3.4. Cyclotomic structures on the $E_{8}$ root lattice. In this section $W=$ $W\left(E_{8}\right)$ is the Weyl group of the root system $R$ of type $E_{8}$ and $X=\mathbb{Z} R$ is the $E_{8}$ root lattice. There is exactly one cyclotomic class in $W$ of every order

$$
d \in\{1,2,3,4,5,6,8,10,12,15,20,24,30\} .
$$

In this section we determine the $K$-equivalence classes $S$ and the orders of the subgroups $C_{S}(w)$ for each class of cyclotomic elements $w \in W$. We thereby find the number $N$ of reflections in $C(w)$, along with its Shephard-Todd classification. Since $N=\sum\left(d_{i}-1\right)$, where the $d_{i}$ are the degrees of $C(w)$, we can compare our results with those of Springer [35. We ignore the classes of odd order $d$, since their negatives have the same centralizer. If $d=2$, then $w=-1$, so $C(w)=W$. If $d \in\{20,24,30\}$, we have $[K: \mathbb{Q}]=\phi(d)=8$ so $S=R$ and $C(w)=\langle w\rangle$. The nontrivial cases are as follows. 
3.4.1. $d=4$. Here $w$ belongs to the class $D_{4}\left(a_{1}\right)^{2}$ and $w^{2}=-1$. This implies that $\langle\alpha, w \alpha\rangle=0$ for all $\alpha \in R$. Hence all $K$-equivalence classes have type $A_{1}^{2}$ and $C_{S}(w)=\left\langle w_{S}^{2}\right\rangle$ has order two. There are $240 / 4=60 \mathrm{~K}$-equivalence classes, each contributing a single $K$-reflection to $C(w)$, so $N=60$. We note that $60=$ $7+11+19+23$, in accordance with 35 .

3.4.2. $d=6$. Here $w$ belongs to the class $E_{8}\left(a_{8}\right)$. By Lemma 3.11, there are 40 $K$-equivalence classes $S$, each of type $A_{2}$, and each $C_{S}(w)$ is cyclic of order three, giving a total of $N=80 \mathrm{~K}$-reflections in $W_{K}$. The roots in $S$ are the vertices of a planar hexagon and form a single orbit under $\langle w\rangle$ (cf. section A.3 below). We note that $80=11+17+23+29$, in accordance with [35], 1

3.4.3. $d=10$. Here $w$ belongs to the class $E_{8}\left(a_{6}\right)$. By Lemma 3.11 there are 12 $K$-equivalence classes $S$, each of type $A_{4}$, consisting of two $w$-orbits. Each $C_{S}(w)$ is cyclic of order five, generated by a Coxeter element in $W(S)$, giving a total of $N=48 K$-reflections in $C(w)$. We note that $48=19+29$, in accordance with 35.

3.4.4. $d=8$. Here $w$ belongs to the class $D_{8}\left(a_{3}\right)$ and $w^{4}=-1$. We have $c \phi(e)=$ $\phi(8)=4$. Table 2 implies that $S$ has type $A_{1}^{4}$ or $D_{4}$. To analyze this dichotomy, we first make some preliminary remarks on subsystems of type $A_{1}^{4}$ in $R$, which we call tetrads. We say that a tetrad $T= \pm\left\{\alpha_{0}, \alpha_{1}, \alpha_{2}, \alpha_{3}\right\}$ is even if $\alpha_{0}+\alpha_{1}+\alpha_{2}+\alpha_{3} \in 2 X$, and $T$ is odd otherwise. In [6, Lemma 11], Carter proves:

Lemma 3.12. Let $R$ be a root system of type $E_{8}$ with Weyl group $W$. The even and odd tetrads in $R$ each form a single orbit under $W$. The even tetrads are precisely those which are contained in a subsystem of type $D_{4}$.

We return now to our cyclotomic element $w \in W$ of order $d=8$. Every $K$ equivalence class $S$ contains a unique $w$-stable tetrad. This is clear if $S \simeq A_{1}^{4}$ is itself a tetrad. If $S \simeq D_{4}$, then $w$, having order eight, must act on $S$ as a Coxeter element $w_{S} \in W\left(B_{4}\right)$. It follows that are three $w$-orbits on $S$. It is easy to check that these orbits are classified by the value of $\langle\alpha, w \alpha\rangle \in\{-1,0,+1\}$. The orbit $\{\alpha \in S:\langle\alpha, w \alpha\rangle=0\}$ is the unique $w$-stable tetrad in $S$. Let us define

$$
\varsigma:=1+w+w^{-1} \in \operatorname{End}(V) .
$$

If $\beta \in S$ satisfies $\langle\beta, w \beta\rangle=-1$, then $\langle\varsigma \beta, w \varsigma \beta\rangle=+1$. Hence the $w$-orbits in $S$ are represented by $\{\alpha, \beta, \varsigma \beta\}$ for any choice of roots $\alpha, \beta$ in $S$ such that $\langle\alpha, w \alpha\rangle=0$, $\langle\beta, w \beta\rangle=-1$.

To count the $K$-equivalence classes of each type, we must look at the roots in a more explicit way. As in [3], the roots of $R$ are the vectors

$$
e_{i} \pm e_{j}, \quad \frac{1}{2} \sum c_{i} e_{i}
$$

in $\mathbb{R}^{8}$, where $1 \leq i \neq j \leq 8$ and $c_{i} \in\{ \pm 1\}$ with $\prod c_{i}=+1$. The pairing $\langle$,$\rangle is then$ the usual dot product on $\mathbb{R}^{8}$. For visual clarity, we use an abbreviated notation for roots of the form $\frac{1}{2} \sum c_{i} e_{i}$, as in the following example:

$$
\frac{1}{2}(1,-1,-1,1,1,-1,1,-1)=[+--+\mid+-+-] .
$$

\footnotetext{
${ }^{1}$ In [35 5.4], the degree $d_{4}=30$ is omitted by mistake in the row for $d=6$ but it appears in the row for $d=3$, which has the same centralizer.
} 
The roots of the form $e_{i} \pm e_{j}$ comprise a root subsystem $R^{\prime}$ of $R$ of type $D_{8}$. We choose $w \in W\left(R^{\prime}\right)$ such that

$$
w: e_{1} \mapsto e_{2} \mapsto e_{3} \mapsto e_{4} \mapsto-e_{1}, \quad e_{5} \mapsto e_{6} \mapsto e_{7} \mapsto e_{8} \mapsto-e_{5} .
$$

Using the criteria in 3.12, we find there are $18 w$-stable tetrads in $R$; twelve of these tetrads are odd and six of them are even. The twelve $K$-equivalence classes $S \simeq A_{1}^{4}$ are the $w$-orbits through the following twelve roots $\alpha$ :

$$
\begin{array}{cc}
e_{1} \pm e_{6}, & e_{1} \pm e_{8}, \\
{[++++\mid \pm \mp \pm \mp],} & {[ \pm \mp \pm \mp \mid++++],} \\
{[++++\mid \pm \mp \mp \pm],} & {[ \pm \mp \mp \pm \mid++++] .}
\end{array}
$$

The six $K$-equivalence classes $S \simeq D_{4}$ are each the union of three $w$-orbits, through $\alpha, \beta, \varsigma \beta$, with $\langle\alpha, w \alpha\rangle=0,\langle\beta, w \beta\rangle=-1$, as shown:

\begin{tabular}{|c|c|c|}
\hline$\alpha$ & $\beta$ & $\varsigma \beta$ \\
\hline \hline$e_{1}-e_{3}:$ & $e_{1}-e_{2}$ & $-e_{3}-e_{4}$ \\
\hline$e_{5}-e_{7}:$ & $e_{5}-e_{6}$ & $-e_{7}-e_{8}$ \\
\hline$e_{1} \pm e_{5}:[+-+-\mid \pm \mp \pm \mp]$ & {$[++--\mid \pm \pm \mp \mp]$} \\
\hline$e_{1} \pm e_{7}:[+-+-\mid \mp \pm \pm \mp]$ & {$[++--\mid \pm \pm \pm \pm]$} \\
\hline
\end{tabular}

If $S=4 A_{1}$ is a tetrad, then $C_{S}(w)=\{ \pm 1\}$ has order two. If $S=D_{4}$, then $C_{S}(w)=\left\langle w_{S}^{2}\right\rangle$ has order four. Thus, there are $N=12 \cdot 1+6 \cdot 3=30$ reflections in $C(w)$. We note that $30=7+23$, in accordance with 35 .

The determinant det $: U\left(V_{K}, h\right) \rightarrow K^{\times}$maps $C(w)$ onto $\left\langle w^{2}\right\rangle$, with kernel $C(w)_{1}=C(w) \cap S U\left(V_{K}, h\right)=\tilde{O}$, the binary octahedral group, which contains the binary tetrahedral group $\tilde{T}=Q_{8} \cdot 3$ with index two. Thus, $C(w)=\langle w\rangle \cdot \tilde{O}$. The six reflections of type $A_{1}^{4}$ are the elements $w^{2} \cdot\{ \pm i, \pm j, \pm k\} \subset w^{2} \cdot Q_{8}$. The twelve reflections of order four are the elements $\pm w \cdot x$ where $x \in \tilde{O}-\tilde{T}$ has an eigenvalue equal to $\pm w^{-1}$ on $V_{K}$.

3.4.5. $d=12$. Here $w$ belongs to the class $E_{8}\left(a_{3}\right)$. We have $c \phi(e)=\phi(12)=4$. Using Table 2 we find two possibilities for a $K$-equivalence class: $S=A_{2}^{2}$ or $S=D_{4}$. This time, the orbit invariant

$$
\langle\alpha, w \alpha\rangle \in\{-1,0,+1\}
$$

determines the isomorphism type of $S$. Indeed, for any $\alpha \in R$, the relation $w^{4}-$ $w^{2}+1=0$ implies that

$$
\left\langle\alpha, w^{2} \alpha\right\rangle=2+\left\langle\alpha, w^{4} \alpha\right\rangle
$$

Since $w^{2} \alpha \neq \alpha \neq-w^{4} \alpha$ we must have $\left\langle\alpha, w^{2} \alpha\right\rangle=1$. Writing the relation as $w^{3}-w+w^{-1}=0$ shows that

$$
\left\langle\alpha, w^{3} \alpha\right\rangle=\langle\alpha, w \alpha\rangle-\left\langle\alpha, w^{-1} \alpha\right\rangle=0 .
$$

If $\langle\alpha, w \alpha\rangle=0$, then $S$ contains, hence coincides with $A_{2}^{2}$ and has root basis

$$
\left\{\alpha,-w^{2} \alpha\right\} \cup\left\{w \alpha,-w^{3} \alpha\right\}
$$

for the two $A_{2}$ components. Hence $|S|=12$ and consists of a single $w$-orbit. We have $c=2$ and $w^{2}$ acts as the graph automorphism on each component of $S$. The group $C_{S}(w)=\left\langle w_{S}^{4}\right\rangle$ has order three. 
TABLE 3. Reflection groups $C(w)$ for cyclotomic $w \in W\left(E_{8}\right)$

\begin{tabular}{|c|c|c|c|c|c|}
\hline$d:$ & 4 & 3,6 & 8 & 5,10 & 12 \\
\hline \hline class & $D_{4}\left(a_{1}\right)^{2}$ & $A_{2}^{4}, E_{8}\left(a_{8}\right)$ & $D_{8}\left(a_{3}\right)$ & $A_{4}^{2}, E_{8}\left(a_{6}\right)$ & $E_{8}\left(a_{3}\right)$ \\
\hline$|C(w)|:$ & $8 \cdot 12 \cdot 20 \cdot 24$ & $12 \cdot 18 \cdot 24 \cdot 30$ & $8 \cdot 24$ & $20 \cdot 30$ & $12 \cdot 24$ \\
\hline $\operatorname{dim} V_{K}$ & 4 & 4 & 2 & 2 & 2 \\
\hline type of $S$ & $2 A_{1}$ & $A_{2}$ & $A_{1}^{4}, D_{4}$ & $A_{4}$ & $A_{2}^{2}, D_{4}$ \\
\hline$\left|C_{S}(w)\right|$ & 2 & 3 & 2,4 & 5 & 3,4 \\
\hline number of $S$ & 60 & 80 & 12,6 & 12 & 8,6 \\
\hline$N$ & $2^{60}$ & $3^{80}$ & $2^{18} 4^{12}$ & $5^{48}$ & $2^{6} 3^{16} 4^{12}$ \\
\hline ST number & 31 & 32 & 9 & 16 & 10 \\
\hline
\end{tabular}

If $\langle\alpha, w \alpha\rangle=1$, then $S=D_{4}$ with root basis

$$
\left\{w \alpha-\alpha, \quad w^{2} \alpha-w \alpha, \quad w \alpha-w^{3} \alpha, \quad \alpha-w^{2} \alpha+w^{3} \alpha\right\},
$$

where $w^{2} \alpha-w \alpha$ corresponds to the branch node. Now $|S|=24$ so $S$ consists of two $w$-orbits. The orbit not containing $\alpha$ satisfies $\langle\beta, w \beta\rangle=-1$. Here $w_{S}$ is a Coxeter element in $W\left(F_{4}\right)=A\left(D_{4}\right)$, whose image in $A\left(D_{4}\right) / W\left(D_{4}\right)$ is a triality. The group $C_{S}(w)=\left\langle w_{S}^{3}\right\rangle$ has order four.

We can count the number of $K$-equivalence classes of each type, by invoking Springer's results instead of verifying them. Let $a$ and $b$ be the number of $K$ equivalence classes of type $2 A_{2}$ and $D_{4}$, respectively. Counting $w$-orbits in each type, we have one equation: $a+2 b=240 / 12=20$. On the other hand, The degrees of $E_{8}$ are $2,8,12,14,18,20,24,30$, so by 35 the degrees of $C(w)$ are 12,24 , and there are $N=11+23=34$ reflections in $C(w)$. Counting the number of reflections in each group $C_{S}(w)$, we get a second equation $2 a+3 b=34$. It follows that $a=8$ and $b=6$.

Table 3 summarizes the cases where $W \neq C(w) \neq\langle w\rangle$. Row "number of $S$ " gives the number of $K$-equivalence classes $S$ of each type. Row " $N$ " gives the number of reflections in $C(w)$ of each order. For example, when $d=8$ there are 18 reflections of order two and 12 reflections of order four. The last row gives the label for $C(w)$ according to the Shephard-Todd classification [33.

\section{Coinvariants of Cyclotomic lattices}

Return to a general irreducible root system $R$ with Weyl group $W$ and root lattice $X=\mathbb{Z} R$. For any elliptic $w \in W$, with coinvariants $X_{w}=X /(1-w) X$, we have $\left|X_{w}\right|=\operatorname{det}(1-w)$, but this does not determine the abelian group $X_{w}$ completely. However, if $w$ is cyclotomic, the group $X_{w}$ has a simple description:

Lemma 4.1. Suppose $w \in W$ is cyclotomic of order $d$. If $d$ is not a prime power, then $X_{w}=0$. If $d$ is a power of a prime $p$, then $X_{w}$ is a vector space over $\mathbb{F}_{p}$ of dimension $n / \phi(d)$, where $n=\operatorname{dim} V$.

Proof. This follows from the elementary fact that $\Phi_{d}(1)=1$ unless $d$ is a power of a prime $p$, in which case $\Phi_{d}(1)=p$. Since $m=\Phi_{d}(1)$ kills $X_{w}$, this proves the lemma. 
TABLE 4. The coinvariant representation for elliptic trialities

\begin{tabular}{|c|c|c|c|}
\hline$R$ & $\left|C_{A}(w)\right|$ & $S p\left(X_{w}\right)$ & $\left|S p\left(X_{w}\right)\right|$ \\
\hline \hline$A_{2}$ & $2 \cdot 3$ & $\mathbb{F}_{3}^{\times}$ & $3-1$ \\
\hline$G_{2}$ & 6 & $\mathbb{F}_{3}^{\times}$ & $3-1$ \\
\hline$F_{4}$ & $6 \cdot 12$ & $S L_{2}(3)$ & $3\left(3^{2}-1\right)$ \\
\hline$E_{6}$ & $2 \cdot 6 \cdot 9 \cdot 12$ & {$\left[\mathbb{F}_{3}^{\times} \times S L_{2}(3)\right] \ltimes \mathbb{F}_{3}^{2}$} & $2 \cdot 3^{3}\left(3^{2}-1\right)$ \\
\hline$E_{8}$ & $12 \cdot 18 \cdot 24 \cdot 30$ & $S p_{4}(3)$ & $3^{4}\left(3^{4}-1\right)\left(3^{2}-1\right)$ \\
\hline
\end{tabular}

More suggestively, suppose that $w$ is cyclotomic of order $d$ a power of a prime $p$. Then $\mathfrak{O}=\mathbb{Z}[w]$ is the ring of integers in the cyclotomic field $K=\mathbb{Q}(w) \subset \operatorname{End}(V)$. The ideal $\mathfrak{P}=(1-w) \mathfrak{O}$ is the unique prime ideal in $\mathfrak{O}$ ramified over $\mathbb{Z}$ and we have $p \mathfrak{O}=\mathfrak{P}^{\phi(d)}$ and $\mathfrak{O} / \mathfrak{P} \simeq \mathbb{F}_{p}$. Let $X_{\mathfrak{O}}$ be the abelian group $X$, viewed as an $\mathfrak{O}$-module. Then we have $X_{w}=X_{\mathfrak{O}} / \mathfrak{P} X_{\mathfrak{O}}$, showing that $X_{w}$ is the reduction modulo $p$ of the $\mathfrak{O}$-lattice $X_{\mathfrak{O}}$. The hermitian form $h(x, y)$ on $V_{K}$ is $\mathfrak{O}$-valued on $X_{\mathfrak{O}}$, and we have $h(x, y) \equiv\langle x, y\rangle_{w} \bmod \mathfrak{P}$.

4.1. Elliptic trialities. It is convenient to give the name triality to any group element of order three. This is the smallest order of an interesting elliptic element $w \in W$.

One can classify elliptic trialities as follows. An elliptic triality $w \in W$ is necessarily cyclotomic: its minimal polynomial is $M(t)=t^{2}+t+1$ and its characteristic polynomial is $\operatorname{det}(t I-w)=\left(t^{2}+t+1\right)^{k}$, where $2 k$, the rank of $R$, must be even. Since $w$ is cyclotomic, it is regular, so there is at most one $W$-conjugacy class of elliptic trialities in $W$, by [35, 4.2]. The connection index $f=[P(R): Q(R)]$ divides $\operatorname{det}(1-w)$, hence must be a power of 3 . It follows that $R$ has one of the types $A_{2}, G_{2}, F_{4}, E_{6}, E_{8}$. Elliptic trialities exist in each of these cases: the prime 3 divides the Coxeter number and does not divide any exponent, so for any Coxeter element $v \in W$, the element $w=v^{h / 3}$ is an elliptic triality (cf. Lemma 3.2).

By Lemma 4.1, the coinvariant group $X_{w}$ is a vector space over $\mathbb{F}_{3}$ of dimension equal to half the rank of $R$. Since $3=(1-w)(2+w)$, we have $\dot{M}(t)=2+t$ and the corresponding symplectic form on $X_{w}$ is given by

$$
\left\langle\rho_{\lambda}, \rho_{\mu}\right\rangle_{w}=\langle\lambda,(2+w) \mu\rangle \bmod 3,
$$

in the notation of section 2.1 .

The order of $C(w)$ is the product of the degrees of $W$ which are divisible by 3. Multiplying by $[A: W]$ gives the order of the centralizer $C_{A}(w)$ of $w$ in the automorphism group $A$ of $R$. These are given in Table 4, along with a concrete description of the group $S p\left(X_{w}\right)$ and its order.

In each case, we have

$$
\left|C_{A}(w)\right|=3\left|S p\left(X_{w}\right)\right| .
$$

This suggests the following result. 
Proposition 4.2. If $w$ is an elliptic triality, then the coinvariant representation $\varrho_{w}: C_{A}(w) \rightarrow S p\left(X_{w}\right)$ is a surjective three-fold covering with kernel generated by $w$.

Proof. In view of (21), it suffices to prove that the kernel of $\varrho_{w}$ is generated by $w$. We require two lemmas.

Lemma 4.3. Suppose $w \in W$ is an elliptic triality, let $\lambda \in X$ and set $\delta=(1-w) \lambda$. Then

$$
2\langle w \lambda, \lambda\rangle=-\langle\lambda, \lambda\rangle \quad \text { and } \quad\langle\delta, \delta\rangle=3\langle\lambda, \lambda\rangle \in 3 \mathbb{Z} .
$$

Proof. Since $w+w^{-1}=-1$, we have

$$
2\langle w \lambda, \lambda\rangle=\langle w \lambda, \lambda\rangle+\left\langle\lambda, w^{-1} \lambda\right\rangle=\langle w \lambda, \lambda\rangle+\left\langle w^{-1} \lambda, \lambda\right\rangle=-\langle\lambda, \lambda\rangle .
$$

It follows that

$$
\langle\delta, \delta\rangle=\langle(1-w) \lambda,(1-w) \lambda\rangle=2\langle\lambda, \lambda\rangle-2\langle w \lambda, \lambda\rangle=3\langle\lambda, \lambda\rangle \in 3 \mathbb{Z},
$$

as claimed.

Lemma 4.4. Suppose $\alpha, \beta \in R$ are short roots. Let $w \in W$ be an elliptic triality, and suppose $\alpha, \beta$ have the same class in $X_{w}$. Then $\beta=w^{i} \alpha$ for some $i=0,1,2$.

Proof. Our normalization (10) implies that $\langle\alpha, \alpha\rangle=\langle\beta, \beta\rangle=2$. We are assuming the element $\delta=\alpha-\beta$ vanishes in $X_{w}$, so there is $\lambda \in X$ such that

$$
\delta=(1-w) \lambda,
$$

and we can apply Lemma 4.3

$$
\langle\delta, \delta\rangle=3\langle\lambda, \lambda\rangle, \quad 2\langle w \lambda, \lambda\rangle=-\langle\lambda, \lambda\rangle .
$$

On the other hand, since $\delta=\alpha-\beta$, we have

$$
\langle\delta, \delta\rangle=4-2\langle\alpha, \beta\rangle \in 3 \mathbb{Z} .
$$

Since $\langle\alpha, \beta\rangle \in \mathbb{Z},[3$, VI.1.3] implies

$$
\langle\alpha, \beta\rangle \in\{0, \pm 1, \pm 2\} .
$$

However, equation (23) limits the possibilities to:

$$
\langle\alpha, \beta\rangle \in\{-1,2\} .
$$

If $\langle\alpha, \beta\rangle=2$, then $\alpha=\beta$. Hence from now on we assume $\langle\alpha, \beta\rangle=-1$, which means

$$
\langle\delta, \delta\rangle=6 \quad \text { and } \quad\langle\lambda, \lambda\rangle=2 .
$$

But a vector in $X$ of norm equal to that of a short root is itself a root 20, Prop. $5.10 \mathrm{a})$ ]. Thus, $\lambda$ is also a short root and we have

$$
\langle w \lambda, \lambda\rangle=-\frac{1}{2}\langle\lambda, \lambda\rangle=-1 \text {. }
$$

This implies that

$$
\langle\lambda, \alpha\rangle-\langle\lambda, \beta\rangle=\langle\lambda, \delta\rangle=\langle\lambda,(1-w) \lambda\rangle=\langle\lambda, \lambda\rangle-\langle\lambda, w \lambda\rangle=3 .
$$

But $\lambda, \alpha, \beta$ are roots of the same length, so as above, we have

$$
\langle\lambda, \alpha\rangle,\langle\lambda, \beta\rangle \in\{0, \pm 1, \pm 2\} .
$$

Since $\langle\lambda, \alpha\rangle-\langle\lambda, \beta\rangle=3$, there are two possibilities:

$$
\langle\lambda, \alpha\rangle=2 \quad \text { and } \quad\langle\lambda, \beta\rangle=-1
$$


or

$$
\langle\lambda, \alpha\rangle=1 \quad \text { and } \quad\langle\lambda, \beta\rangle=-2 .
$$

The first possiblity (24) implies that $\lambda=\alpha$, so (22) reads as

$$
\alpha=\beta+(1-w) \alpha,
$$

that is,

$$
\beta=w \alpha .
$$

Likewise, the second possibility implies that $\alpha=w \beta$. The lemma is proved.

Now we can prove Proposition 4.2. Suppose that $u \in C_{A}(w)$ acts trivially on $X_{w}$. Then for every short root $\alpha \in R$, the roots $\alpha$ and $u \alpha$ have the same image in $X_{w}$. Lemma 4.4 implies that $\alpha$ and $u \alpha$ are in the same $w$-orbit. Hence $u$ preserves each $K$-equivalence class $S$ containing a short root in $R$, where $K$ is the subfield of $\operatorname{End}(V)$ generated by $w$. This means that $u$ preserves the $K$-line in $V_{K}$ through $S$. It follows that $u$ commutes with $C_{S}(w)$. The short roots span $V$, so $u$ has all of its eigenvalues in $K$. But the subgroup of $C(w)$ generated by the $C_{S}(w)$ for $S$ containing a short root is irreducible on $V_{K}$, by Corollary 3.10. Hence $u$ acts on $V_{K}$ by a scalar in $K$. The roots of unity in $K$ are generated by $-w$. Since -1 acts nontrivially on $X_{w}$, we see that $u \in\langle w\rangle$, as claimed.

We have shown that for any elliptic triality, the coinvariant representation $\varrho_{w}$ gives an exact sequence

$$
1 \longrightarrow\langle w\rangle \longrightarrow C_{A}(w) \longrightarrow S p\left(X_{w}\right) \longrightarrow 1 .
$$

If $R \neq E_{6}$, the dimension of $V_{K}$ is not divisible by 3 , so the subgroup $C_{A}(w)^{\prime}$ of $C_{A}(w)$ of determinant one on $V_{K}$ is a complement to $\langle w\rangle$ and we have

$$
C_{A}(w)=\langle w\rangle \times S p\left(X_{w}\right) .
$$

All elliptic trialities may be seen in $E_{8}$ : for $R=G_{2}, F_{4}, E_{6}, E_{8}$, let us write $C_{R}(w)=$ $C_{A(R)}(w)$. If $w \in W\left(E_{8}\right)$ is an elliptic triality, then we can write $w=x y z$, where

$$
x \in W\left(G_{2}\right), \quad x y \in W\left(F_{4}\right) \subset W\left(E_{6}\right)
$$

are elliptic trialities in the respective groups and

$$
C_{G_{2}}(x)=C_{G_{2}}(w), \quad C_{F_{4}}(x y)=C_{F_{4}}(w), \quad C_{E_{6}}(x y)=C_{E_{6}}(w) .
$$

Since $w \notin C_{E_{6}}(w)$, the projection of $C_{E_{8}}(w)$ into $S p\left(X_{w}\right)=S p_{4}(3)$ is injective on the groups $C_{R}(w)$, for $R=G_{2}, F_{4}, E_{6}$. Their images give the following chain of algebraic subgroups of $S_{4}(3)$ (with respect to a basis of $X_{w}$ making the matrix of the form $\langle,\rangle_{w}$ antidiagonal):

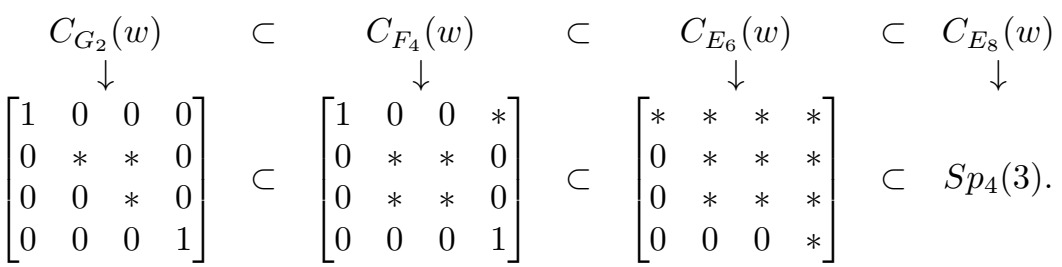

Here $*$ represents arbitrary independent elements of $\mathbb{F}_{3}$ such that the indicated matrix preserves the form $\langle,\rangle_{w}$. Hence, for $R=E_{6}$, the sequence (26) does not split. We will revisit elliptic trialities in Appendix $\mathrm{A}$. 
4.2. Cyclotomic centralizers and coinvariants for $E_{8}$. In this section we analyze the coinvariant representations for the remaining cyclotomic classes in type $E_{8}$. Recall that there is exactly one $W$-conjugacy class of cyclotomic elements in $W$ for each order $d \in\{1,2,3,4,5,6,8,10,12,15,20,24,30\}$. To be elliptic, we of course need $d \geq 2$. The case $d=2$ is well known (cf. the introduction) and $d=3$ was covered in the previous section. Henceforth we only consider $d \geq 4$. We ignore $d=6,10$ since $X_{w}=0$ and $-w$ has the same centralizer with $X_{-w} \neq 0$. We also ignore those $d$ where $C(w)=\langle w\rangle$, since there is nothing to say in these cases. For $d=12$ we have $|C(w)|=288$ but $X_{w}=0$. See, however 4.2 .4 for a modular interpretation of this centralizer.

What remain are $d=4,5,8$. In these cases the group $\operatorname{Sp}\left(X_{w}\right)$ is the isometry group of a nondegenerate symplectic form over a finite field. The following result has been proved for $d=3$.

Proposition 4.5. If $W$ has type $E_{8}$ and $w \in W$ is cyclotomic of order $d \geq$ 3 a prime power, then the coinvariant representation $\varrho_{w}: C(w) \rightarrow S p\left(X_{w}\right)$ is surjective.

We prove this by examining $d=4,5,8$ separately.

4.2.1. Coinvariants for $d=5$. This section contains the proof of Proposition 4.5 for $d=5$. We will see that a sharper result holds:

$$
C(w)=\langle w\rangle \times S L_{2}(5) .
$$

The field $K=\mathbb{Q}(w)$ is generated by fifth roots of unity and $\mathfrak{P}=(1-w) \mathfrak{O}$ is the ramified prime in the ring of integers $\mathfrak{O}=\mathbb{Z}[w]$. Let $h$ be the invariant hermitian form on $X_{\mathfrak{O}}$, as in section 4. For any $K$-equivalence class $S \subset R$, the $K$-reflection $r_{S}$ acts on $X_{w}=X_{\mathfrak{O}} / \mathfrak{P} X_{\mathfrak{O}} \simeq \mathbb{F}_{5}^{2}$ by

$$
r_{S}(x)=x-h(x, \alpha) \rho_{\alpha}=x-\langle x, \alpha\rangle_{w} \rho_{\alpha},
$$

where $\alpha \in S$ and $\rho_{\alpha}$ denotes the image of $\alpha$ in $X_{w}$. Since the pairing $\langle,\rangle_{w}$ is nondegenerate on $X_{w}$, there are two roots $\alpha, \beta$ such that $\left\langle\rho_{\alpha}, \rho_{\beta}\right\rangle_{w} \neq 0 \bmod 5$ and $\left\{\rho_{\alpha}, \rho_{\beta}\right\}$ is a basis of $X_{w}$. Letting $S, T$ be the $K$-equivalence classes of $\alpha, \beta$, the coinvariant representation is given in terms of this basis by

$$
\varrho_{w}\left(r_{S}\right)=\left[\begin{array}{cc}
1 & \left\langle\rho_{\beta}, \rho_{\alpha}\right\rangle_{w} \\
0 & 1
\end{array}\right], \quad \varrho_{w}\left(r_{T}\right)=\left[\begin{array}{cc}
1 & 0 \\
\left\langle\rho_{\alpha}, \rho_{\beta}\right\rangle_{w} & 1
\end{array}\right] .
$$

Hence $\varrho_{w}\left(r_{S}\right)$ and $\varrho_{w}\left(r_{T}\right)$ generate $S L_{2}(5)$, proving surjectivity of $\varrho_{w}$ for $d=5$. Since $|C(w)|=600=5 \cdot\left|S L_{2}(5)\right|$, this proves Proposition 4.5 for $d=5$. Since $w$ has nontrivial determinant on $V_{K}$ we have $C(w)=\langle w\rangle \times S p\left(X_{w}\right)=\langle w\rangle \times S L_{2}(5)$, as claimed.

4.2.2. Coinvariants for $d=8$. This section contains the proof of Proposition 4.5 for the case $d=8$, where $w$ belongs to the cyclotomic class $D_{8}\left(a_{3}\right)$, with minimal polynomial $M(t)=t^{4}+1$. The field $K=\mathbb{Q}(w)$ is generated by the eighth roots of unity. Recall that each $K$-equivalence class $S$ contains a root $\alpha \in S$ such that $\langle\alpha, w \alpha\rangle=0$ and that $\left\{\alpha, w \alpha, w^{2} \alpha, w^{3} \alpha\right\}$ is a tetrad. Now $\dot{M}(w)=1+w+w^{2}+w^{3}$ and $S \simeq A_{1}^{4}$ or $S \simeq D_{4}$. By Lemma 3.12, the latter holds iff $\dot{M}(w) \alpha \in 2 X$. Since $2=(1-w) \dot{M}(w)$, we have $S \simeq D_{4}$ precisely when $\alpha \in(1-w) X$, meaning that $\rho_{\alpha}=0$. So the roots in an even tetrad vanish in $X_{w}$ and the roots in an odd tetrad do not vanish. The three nonzero vectors in $X_{w}$ are the images of 
$e_{1}+e_{6},[++++\mid+-+-]$ and $[++++\mid+--+]$. We have $h(\alpha, \alpha)=2$, so formula (27) holds in this case as well, and the same argument shows that $\operatorname{im} \varrho_{w}=S p\left(X_{w}\right)=S L_{2}(2)=S_{3}$.

In section 3.4 .4 we saw that $\varrho_{w}$ is surjective on the hyperoctahedral group $\tilde{O}$. Since $Q_{8}$ is the unique normal subgroup of $\tilde{O}$ with quotient $S_{3}$, it follows that ker $\varrho_{w}$ is a central product $\langle w\rangle \cdot Q_{8}$ of order 32 .

4.2.3. Coinvariants for $d=4$. This section contains the proof of Proposition 4.5 for the case $d=4$. Let $\bar{X}=X / 2 X$, and let $O(\bar{X})$ be the orthogonal group of the quadratic form $q=\frac{1}{2}\langle x, x\rangle \bmod 2$ on $\bar{X}$. The map $W \rightarrow O(\bar{X})$ sends $w$ to an involution $\bar{w} \in O(\bar{X})$ and the projection $X \rightarrow \bar{X}$ induces an isomorphism $\bar{X}_{w} \simeq X_{w}$ on coinvariants. Let $\bar{X}^{w}$ denote the invariants of $w$ in $\bar{X}$. Since $\operatorname{dim} \bar{X}^{w}=$ $\operatorname{dim} \bar{X}_{w}=4$ it follows that $\bar{X}^{w}=(1-w) \bar{X}$, which implies that $\bar{X}^{w}$ is a maximal $q$-isotropic subspace of $\bar{X}$. The subgroup $U \subset O(\bar{X})$ acting trivially on $\bar{X}^{w}$ also acts trivially on $\bar{X}_{w}$ and is the unipotent radical of the parabolic subgroup in $O(\bar{X})$ with Levi $G L_{4}(2)$. It follows that $\operatorname{ker} \varrho_{w}=\tilde{U} \cap C(w)$, where $\tilde{U}$ is the pre-image of $U$ in $W$.

The centralizer $C_{O(\bar{X})}(\bar{w})$ surjects onto $S p\left(\bar{X}_{w}\right)$ with kernel $U$, and the preimage of $C_{O(\bar{X})}(\bar{w})$ in $W$ is the normalizer $N(w)=\left\{v \in W: w^{v}=w^{ \pm 1}\right\}$, since $w^{2}=-1$. One can check that $N(w)$ preserves the form $\langle,\rangle_{w}$. Hence the coinvariant representation $\varrho_{w}$ extends to a surjection $N(w) \rightarrow S p\left(X_{w}\right)$ with kernel $\tilde{U}$. To see that $\varrho_{w}$ is surjective on $C(w)$, it remains only to show that $\tilde{U}$ is not contained in $C(w)$. This can be done by a direct computation: The element $w$, viewed in $W\left(D_{8}\right)$, is a product of four commuting $B_{2}$-Coxeter elements of the form $(i j) t_{j}$, where $(i j)$ is a transposition and $t_{j}$ is a sign change. One easily finds a permutation $z$ inverting $w$, such that $\rho_{w}(z) \neq 1$, using Lemma 2.4. Thus, we have shown that $\varrho_{w}$ is surjective.

It follows that ker $\varrho_{w}$ has order 64 . To find its structure, let $x_{i}$ be the square of each $B_{2}$-Coxeter element in $w$, for $i=1,2,3,4$. Each $x_{i}$ belongs to $C(w)$. Using Lemma 2.4. one checks that $x_{i} \in \operatorname{ker} \varrho_{w}$ for each $i$. Along with $w$, these elements $x_{i}$ generate a subgroup $A \simeq\left(C_{4} \times C_{2}^{4}\right) / \Delta C_{2}$ of index $2^{2}$ in ker $\varrho_{w}$. Additional computation in $W_{D_{8}}$ shows that ker $\varrho_{w} \simeq A \rtimes K_{4}$, where the Klein four-group $K_{4}$ acts on $A$ by permuting the coordinates.

4.2.4. Coinvariants for $d=12$. Here $X_{w}=0$, so the coinvariant representation gives no information about $C(w)$. However, $w^{4}$ is an elliptic triality, so we have

$$
C(w) \subset C\left(w^{4}\right)=\left\langle w^{4}\right\rangle \times S p_{4}(3),
$$

via the coinvariant representation of $C\left(w^{4}\right)$. Note that $w$ is linear over the field $K=\mathbb{Q}\left(w^{4}\right)$, with $\operatorname{det}\left(t-w \mid V_{K}\right)=\Phi_{12}(t)$, which reduces to $\Phi_{4}^{2}$ on $X_{w^{4}}$. Hence the centralizer of $w$ in $S p_{4}(3)$ is $U_{2}(3)$. This shows that

$$
C(w)=\left\langle w^{4}\right\rangle \times C_{S p_{4}(3)}\left(w^{4}\right) \simeq C_{3} \times U_{2}(3) .
$$

\section{Noncyclotomic Classes in $E_{8}$}

5.1. The abelian group $X_{w}$ and orbit types. The group $X_{w}$ has cardinality $\left|X_{w}\right|=\operatorname{det}(1-w)$, but in the noncyclotomic cases, the group structure of $X_{w}$ is not immediately evident. It is helpful to also consider the subgroup $\hat{T}^{w}$ of fixed points of $w$ in the torus $\hat{T}=X \otimes \mathbb{C}^{\times}$, which may be viewed as a maximal torus in 


\begin{tabular}{|c|c|c|c|c|c|c|c|c|}
\hline type of $W_{t}$ & $D_{8}$ & $A_{1} A_{7}$ & $A_{1} A_{2} A_{5}$ & $A_{4}^{2}$ & $D_{5} A_{3}$ & $E_{6} A_{2}$ & $A_{1} E_{7}$ & $A_{8}$ \\
\hline order of $t$ & 2 & 4 & 6 & 5 & 4 & 3 & 2 & 3 \\
\hline
\end{tabular}

the complex Lie group $\hat{G}$ of type $E_{8}$. Since $E_{8}$ is simply-laced and the lattice $X$ is self-dual, we have $X_{w} \simeq \hat{T}^{w}$ as $C(w)$-modules. If $t \in \hat{T}^{w}$, then $w$ belongs to the stabilizer $W_{t}$, which is a reflection subgroup of $W$, since $\hat{G}$ is simply-connected. As $w$ is elliptic, $W_{t}$ has rank eight. It follows that $t$ belongs to one of the nine conjugacy classes of elements in $\hat{G}$ with semisimple centralizer. These correspond to the nodes of the affine Dynkin diagram; the order of $t$ is equal to the corresponding coefficient of the highest root. This is tabulated for $t \neq 1$ as follows: The $C(w)$-orbit of $t$ has cardinality equal to the index $\left[C(w): C_{t}(w)\right]$, where $C_{t}(w)=C(w) \cap W_{t}$. We define the type of the orbit $C(w) \cdot t$ to be the type of $W_{t}$, as in the table above. It turns out that each orbit type appears at most once in $X_{w}$. We indicated this in Table 1 as (size of orbit)[type of orbit]. To find all the orbit types, one need only check which $W_{t}$ contain a conjugate of $w$.

For example, suppose $w$ belongs to the class $A_{3} D_{5}\left(a_{1}\right)$, where $\left|X_{w}\right|=16$. A conjugate of $w$ appears in two subgroups $W_{t}$, of types $A_{3} D_{5}$, and $D_{8}$, with indices $\left[C(w): C_{t}(w)\right]=12$ and 3 , respectively, giving the size of each orbit. In Table 1 we indicate this orbit decomposition of $X_{w}-\{0\}$ as

$$
12\left[A_{3} D_{5}\right]+3\left[D_{8}\right] \text {. }
$$

Since $X_{w}$ has 12 elements of order four and 3 elements of order two, we find that $X_{w} \simeq(\mathbb{Z} / 4 \mathbb{Z})^{2}$.

The orbit types are also important for the structure of $L$-packets; see section 6.8 below.

5.2. Injectivity results for the coinvariant representation. The following observations enable us to show in several cases at once that ker $\varrho_{w}=\langle w\rangle$, and are useful in the more difficult cases below.

Lemma 5.1. Let $W^{\prime}$ be a reflection subgroup of $W=W\left(E_{8}\right)$ generated by reflections corresponding to a maximal subdiagram of the affine diagram of $W$. Suppose $w \in W^{\prime}$ is elliptic and generates its own centralizer in $W^{\prime}$ Then $\operatorname{ker} \varrho_{w}=\langle w\rangle$.

Proof. View $W$ as the Weyl group of a maximal torus $\hat{T}$ in a complex Lie group $\hat{G}$ of type $E_{8}$. Since $\hat{G}$ is simply-laced, simply-connected and adjoint, we have that $W^{\prime}$ is the centralizer in $W$ of some element $t \in \hat{T}^{w}$ and $\hat{T}^{w} \simeq X_{w}$ as $C(w)$-modules. Since ker $\varrho_{w}$ acts trivially on $X_{w}$, it fixes $t$. It follows that $\operatorname{ker} \varrho_{w} \subset W^{\prime} \cap C(w)=\langle w\rangle$. Since the reverse containment is clear, the lemma is proved.

Lemma 5.2. Let $W^{\prime}=W_{1}^{\prime} \times W_{2}^{\prime}$ be a reflection subgroup of $W=W\left(E_{8}\right)$ generated by reflections corresponding to a maximal subdiagram of the affine diagram of $W$ which is the union of two orthogonal subdiagams whose reflections generate $W_{1}^{\prime}$ and $W_{2}^{\prime}$, respectively. Suppose $w=w_{1} w_{2} \in W^{\prime}$ is elliptic, where $w_{i} \in W_{i}^{\prime}$ generates its own centralizer in $W_{i}^{\prime}$ for $i=1,2$. Assume that the order of $\varrho_{w}\left(w_{1}\right)$ divides the order of $w_{2}$. Then $\operatorname{ker} \varrho_{w}=\langle w\rangle$.

Proof. We have $C(w) \cap W^{\prime}=\left\langle w_{1}\right\rangle \times\left\langle w_{2}\right\rangle$. Let $d$ be the order of $\varrho_{w}\left(w_{1}\right)$, so that $\left\langle w_{1}\right\rangle \cap \operatorname{ker} \varrho_{w}=\left\langle w_{1}^{d}\right\rangle$. Then ker $\varrho_{w} \subset\langle w\rangle \cdot\left\langle w_{1}^{d}\right\rangle$. But if $d$ divides the order of $w_{2}$, then $w_{1}^{d}=w^{d}$. Therefore ker $\varrho_{w}=\langle w\rangle$. 
Lemma 5.3. Let $\left\{\beta_{1}, \ldots, \beta_{k}\right\}$ be a nonempty set of orthogonal roots contained in an even tetrad $T$ and let $w=u v$, where $u=r_{\beta_{1}} \cdots r_{\beta_{k}}$ and $v \beta_{i}=\beta_{i}$ for $1 \leq i \leq k$. Then $u$ acts nontrivially on $X_{w}$.

Since $W$ is transitive on even tetrads, we may assume that $T=\left\{\alpha_{2}, \alpha_{4}, \alpha_{8}, \alpha_{2}+\right.$ $\left.\alpha_{4}+\alpha_{8}+2 \alpha_{3}\right\}$. The normalizer of $T$ in the subgroup $\left\langle r_{2}, r_{3}, r_{4}, r_{8}\right\rangle \simeq W\left(D_{4}\right)$ is transitive on $T$. Hence we may assume that $\alpha_{2} \in T$. We have

$$
u \alpha_{1}=\alpha_{1}+\mu,
$$

where $\mu=\sum_{i \in I} \beta_{i}$ and the set $I=\left\{i:\left\langle\alpha_{1}, \beta_{i}\right\rangle \neq 0\right\}$ has cardinality one or two. It suffices to prove that $\mu \notin(1-w) X$.

Suppose $\mu=(1-w) \lambda$, with $\lambda \in X$. Since $w \mu=-\mu$, we have $\dot{M}(w) \mu=\dot{M}(-1) \mu$, where $M(t)$ is the minimal polynomial of $w$ and $\dot{M}(t)=(M(t)-M(1)) / t-1$, as before. Since -1 is an eigenvalue of $w$, we have $M(-1)=0$, so $\dot{M}(-1)=\frac{1}{2} M(1)$ and

$$
\frac{1}{2} M(1) \mu=\dot{M}(w) \mu=\dot{M}(w)(1-w) \lambda=M(1) \lambda,
$$

so that $\mu=2 \lambda$. But then we have

$$
|I|=\frac{1}{2}\langle\mu, \mu\rangle=2\langle\lambda, \lambda\rangle,
$$

implying that 4 divides $|I|$, contradicting $|I| \in\{1,2\}$.

5.3. $A_{1} E_{7}\left(a_{2}\right)$. We now turn to the individual cases not covered by the previous results. Here $w$ lives in the subgroup $W_{t} \simeq W\left(A_{1} E_{7}\right)$ stabilizing an involution $t \in \hat{T}$. More precisely, $w$ is a commuting product $w=u v$, where $u$ is a reflection and $v \in W\left(E_{7}\right)$ has order 12 and centralizer of order 24 . The minimal polynomial of $w$ is $M(t)=\Phi_{12}(t) \Phi_{6}(t) \Phi_{2}(t)$ so $M(1)=2$ and $X_{w} \simeq(\mathbb{Z} / 2 \mathbb{Z})^{2}$. Since $\left|C_{W_{t}}(w)\right|=2 \cdot 24$, so $\left[C(w): C_{W_{t}}(w)\right]=6=\left|S L_{2}(2)\right|$, it follows that $\operatorname{ker} \varrho_{w}=C_{W_{t}}(w)$ and $\varrho_{w}$ is surjective. Moreover, since $\Phi_{2}(t)$ divides $\operatorname{det}(t-v)$, it follows that the long element $w_{7}$ of $W\left(E_{7}\right)$ is not in $\langle v\rangle$. Hence $\operatorname{ker} \varrho_{w}=\langle u\rangle \times\left\langle w_{7}\right\rangle \times\langle v\rangle$ is abelian of type $(2,2,12)$.

5.4. $A_{1} E_{7}\left(a_{4}\right)$. Here $w=-v$, where $v \in W\left(E_{6}\right)$ is in the class $A_{2}^{3}$. Hence $C(w)$ contains a reflection subgroup isomorphic to $W\left(A_{2}\right) \times C_{E_{6}}(v)$. We have $w^{3}=-1$, so $X / 2 X \simeq \mathbb{F}_{2}^{8}$ surjects onto $X_{w} \simeq \mathbb{F}_{2}^{2}$, with kernel equal to the image of $(1-v) X$ in $X / 2 X$. Since $X=Q\left(A_{2}\right)+Q\left(E_{6}\right)$ modulo two, it follows that $(1-v) X=$ $Q\left(E_{6}\right)$ modulo two. Since $W\left(E_{6}\right)$ acts trivially on $X / Q\left(E_{6}\right)$, it follows that $\langle-1\rangle \times$ $C_{E_{6}}(v) \subset \operatorname{ker} \varrho_{w}$. We have seen that $\langle-1\rangle \times C_{E_{6}}(v)$ is the Heisenberg parabolic subgroup of $S p_{4}(3)$, and has order $6^{4}$. On the other hand, direct computation shows that $\varrho_{w}$ is injective on $W\left(A_{2}\right)$. Since $|C(w)|=6^{5}$, it follows that $C(w)=$ $\langle-1\rangle \times C_{E_{6}}(w) \times W\left(A_{2}\right)$ and that $\varrho_{w}$ is a projection onto the factor $W\left(A_{2}\right) \simeq S L_{2}(2)$.

5.5. $A_{2} E_{6}\left(a_{2}\right)$. Here $w^{2}$ has type $A_{2}^{4}$, and $x:=\varrho_{w^{2}}(w)$ is an involution on $X_{w^{2}} \simeq \mathbb{F}_{3}^{4}$ with eigenvalues $1,1,-1,-1$. The natural map $X_{w^{2}} \rightarrow X_{w}$ is a projection onto the +1 -eigenspace of $w$. It follows that

$$
C(w)=\left\langle w^{2}\right\rangle \times C_{S p_{4}(3)}(x)=\left\langle w^{2}\right\rangle \times S L_{2}(3) \times S L_{2}(3),
$$

and that $\rho_{w}$ is a projection onto one of the $S L_{2}(3)$-factors. 
5.6. $A_{1}^{2} D_{6}$. Let $t \in \hat{T}$ be an involution of type $A_{1} E_{7}$. In $W\left(E_{7}\right)$, let $s$ be an involution of type $A_{1} D_{6}$. The centralizer $W_{s, t}$ has type $2 A_{1} D_{6}$ and $w$ is a Coxeter element in $W_{s, t}$. The orders are given by

$$
\left|C_{s, t}(w)\right|=2^{3} \cdot 5, \quad|C(w)|=2^{4} \cdot 3 \cdot 5^{2}, \quad\left|S p_{4}(2)\right|=2^{4} \cdot 3^{2} \cdot 5=720 .
$$

Since ker $\varrho_{w}$ contains an element of order five and $C_{s, t}(w)$ has no elements of order three, the index $k=\left[C_{s, t}(w): \operatorname{ker} \varrho_{w}\right]$ divides $2^{3}$ and $\left|\operatorname{im} \varrho_{w}\right|=30 k$. But $k \neq 8$ since $S p_{4}(2)=S_{6}$ has no subgroup of index three. Hence $k \in\{1,2,4\}$. Lemma 5.3 implies that $k=4$.

Therefore $\left|\operatorname{im} \varrho_{w}\right|=120$ and $\operatorname{im} \varrho_{w}$ is one of the two outer-conjugate subgroups $H, H^{\prime}$ in $S p_{4}(2)$ isomorphic to $S_{5}$. These are distinguished by $H$ containing transvections while $H^{\prime}$ does not. Let $r, r^{\prime}$ denote the reflections generating $W\left(2 A_{1}\right)$ in $C(w)$. Then $\varrho_{w}(r), \varrho\left(r^{\prime}\right)$ are transvections on $X_{w}$, so im $\varrho_{w}=H$.

This $H$ comes from a point-stabilizer in $S_{6}$ under the isomorphism $S_{6} \rightarrow S p_{4}(2)$, which can be seen as follows. Let $\tilde{E}$ be the set of even subsets of $S=\{1, \ldots, 6\}$, with addition given by symmetric difference, and bilinear form $\langle u, v\rangle=|u \cap v| \bmod 2$. The radical is the line $\{\varnothing, S\}$, so $E:=\tilde{E} /\{\varnothing, S\}$ is a nondegenerate symplectic 4 -space over $\mathbb{F}_{2}$. Every nonzero vector in $E$ can be uniquely represented by one of the 15 pairs $[i j]$. A transposition $(i j) \in S_{6}$ becomes the transvection $t_{i j}(v)=$ $v+\langle v,[i j]\rangle[i j]$ on $E$ and $H=\left\langle t_{12}, t_{23}, t_{34}, t_{45}\right\rangle$. The nonzero orbits of $H$ on $E$ are

$$
\{[i j]: 1 \leq i<j \leq 5\}, \quad\{[i 6]: 1 \leq i \leq 5\} .
$$

The stabilizers in $W$ of involutions in $\hat{T}$ are of type $W\left(A_{1} E_{7}\right)$ and $W\left(D_{8}\right)$ and $w$ is contained in groups of both types, where it has centralizers of orders $2^{3} \cdot 3 \cdot 5$ and $2^{4} \cdot 3 \cdot 5$. The orbits in (29) correspond to ten orbits of type $A_{1} E_{7}$ and five orbits of type $D_{8}$.

5.7. $D_{4}^{2}$. Since this class is also $A_{1}^{2} D_{6}\left(a_{2}\right)$, this is similar to the previous case of 5.6. whose notation we keep. We again have $X_{w} \simeq E$, with

$$
\left|C_{s, t}(w)\right|=2^{4} \cdot 3^{2}, \quad|C(w)|=2^{5} \cdot 3^{4}, \quad\left|S p_{4}(2)\right|=\left|S_{6}\right|=2^{4} \cdot 3^{2} \cdot 5,
$$

the index $\ell=\left[C_{s, t}(w): \operatorname{ker} \varrho_{w}\right]$ divides $2^{3}$. We cannot have $\ell=2^{3}$, lest $S_{6}$ have a subgroup of index five. Lemma 5.3 then implies that $\ell=2^{2}$. The image of $\varrho_{w}$ is the subgroup $K=S_{3}^{2} \cdot 2 \subset S_{6}$ of index ten stabilizing a bisection $(a b c)(d e f)$ of $S$. The nonzero orbits of $K$ on $E$ are

$$
\{[i j]: 1 \leq i, j \leq 3 \text { or } 4 \leq i, j \leq 6\}, \quad\{[i j]: 1 \leq i \leq 3 \text { and } 4 \leq j \leq 6\} .
$$

These are six involutions of type $A_{1} E_{7}$ and six involutions of type $D_{8}$, respectively. Computing in $W\left(D_{8}\right)$, we find that the elements

$$
x=w^{2} r_{2} r_{3} r_{2} r_{8}, \quad z=r_{9}\left(r_{4} r_{3} r_{2} r_{3} r_{4}\right)\left(r_{5} r_{4} r_{3} r_{4} r_{5}\right)\left(r_{6} r_{5} r_{4} r_{5} r_{6}\right)
$$

generate an $S_{3} \subset \operatorname{ker} \varrho_{w}$, where $r_{9}$ is the reflection about the highest root in $E_{7}$. It follows that

$$
\operatorname{ker} \varrho_{w}=\langle w\rangle \times\langle x, z\rangle \simeq C_{6} \times S_{3} \text {. }
$$


5.8. $A_{1}^{4} D_{4}$. The minimal polynomial of $w$ is $\Phi_{6} \Phi_{2}^{6}$, so $X_{w} \simeq \mathbb{F}_{2}^{6}$. Since $X_{w} \simeq \hat{T}^{w}$, we can view $X_{w}$ as a $C(w)$-stable subspace of the eight-dimensional $\mathbb{F}_{2}$-space $X_{2}=$ $\hat{T}[2]$, with $W$-invariant form $q(x)=\frac{1}{2}\langle x, x\rangle$ split over $\mathbb{F}_{2}$.

On $X_{2}, w$ has eigenvalues $1^{6}, \zeta, 1+\zeta$, where $\zeta \in \mathbb{F}_{4}$ is a cube root of unity. It follows that the centralizer of $w$ in $O\left(X_{2}, q\right)$ is isomorphic to $O_{6}^{-}(2) \times O_{2}^{-}(2)$, a product of nonsplit orthogonal groups. Since $X_{w}=\left(X_{2}\right)^{w}$, we see that $\varrho_{w}$ is the projection onto $O_{6}^{-}(2)$. Since $O_{2}^{-}(2) \simeq C_{3}$, the kernel of $\varrho_{w}$ has order six, hence is generated by $w$. Since $|C(w)|=2^{8} \cdot 3^{5} \cdot 5$, we have

$$
\left|\operatorname{im} \varrho_{w}\right|=2^{7} \cdot 3^{4} \cdot 5=\left|O_{6}^{-}(2)\right| .
$$

Thus, $\operatorname{im} \varrho_{w} \simeq O_{6}^{-}(2) \subset S p_{6}(2)$, which has two orbits on nonzero vectors in $X_{w}$, determined by the values of the invariant quadratic form $q \mid X_{w}$. We can also think of $O_{6}^{-}(2)=W\left(E_{6}\right)$, via reduction modulo two of the $E_{6}$ root lattice. Hence the $C(w)$-orbits in $X_{w}$ correspond to $W\left(E_{6}\right)$-orbits of involutions in the torus of the adjoint group of type $E_{6}$. In this view there are 36 vectors in $X_{w}$ with $W\left(E_{6}\right)$ stabilizers of type $A_{1} A_{5}$ (for $q=1$ ) and 27 vectors with $W\left(E_{6}\right)$-stabilizer of type $D_{5}($ for $q=0)$. In $W\left(E_{8}\right)$ this gives the orbit structure $36\left[A_{1} E_{7}\right]+27\left[D_{8}\right]$.

5.9. $A_{3} D_{5}\left(a_{1}\right)$. Let $t \in \hat{T}$ have centralizer $W_{t}=W\left(A_{3} D_{5}\right)$. Then $\left|C_{t}(w)\right|=2^{4} \cdot 3$ and $t \in \hat{T}^{w}$ has a $C(w)$-orbit of size $\left[C(w): C_{t}(w)\right]=12$. Since $4 X_{w}=0$ and $X_{w}$ has at least 12 elements of order 4 , it follows that $X_{w} \simeq R \oplus R$, where $R=\mathbb{Z} / 4 \mathbb{Z}$. Hence there are exactly 12 vectors of order four in $X_{w}$, forming a single orbit of type $A_{3} D_{5}$. The three vectors of order two form an orbit of type $D_{8}$.

Since $\langle,\rangle_{w}$ is nondegenerate, there exists a basis $u, v$ of $X_{w}$ such that $\langle u, v\rangle_{w}=1$. By skew symmetry, we have $\langle u, u\rangle_{w},\langle v, v\rangle_{w} \in\{0,2\}$. Subtracting one basis vector from the other if necessary, we can arrange that $\langle u, u\rangle_{w}=\langle v, v\rangle_{w}$. If this value is 2 , one counts eight vectors $u^{\prime} \in X_{w}$ with $\left\langle u^{\prime}, u^{\prime}\right\rangle_{w}=2$, which is incompatible with $C(w)$ having an orbit of size 12 . Hence $\langle u, u\rangle=\langle v, v\rangle=0$, so $S p\left(X_{w}\right)=S L_{2}(R)$ and $\left|S p\left(X_{w}\right)\right|=2^{4} \cdot 3$. Since $C(w)$ is transitive on the 12 vectors of order four in $X_{w}$, we have $S p\left(X_{w}\right)=\operatorname{im} \varrho_{w} \cdot U$, where $U \simeq C_{4}$ is the stabilizer of $t$. But $\rho_{w}\left(C_{t}(w)\right) \subset U$, and a direct computation using Lemma 2.4 shows that $\varrho_{w}$ maps the $A_{3}$-factor in $w$ to an element of order four, so in fact $\rho_{w}\left(C_{t}(w)\right)=U$, implying that $\varrho_{w}$ is surjective, with kernel $\langle w\rangle$.

5.10. $A_{3}^{2} A_{1}^{2}$. Here $w$ can also be viewed as $A_{1}^{4} D_{4}\left(a_{1}\right)$. We have $|C(w)|=2^{11} \cdot 3^{2}$ and $w$ is contained in reflection subgroups

$$
W_{1} \simeq W\left(A_{3} D_{5}\right), \quad W_{2} \simeq W\left(A_{1} E_{7}\right), \quad W_{3} \simeq W\left(D_{8}\right)
$$

with indices

$$
\left[C(w): C_{1}(w)\right]=48, \quad\left[C(w): C_{2}(w)\right]=12, \quad\left[C(w): C_{3}(w)\right]=3 .
$$

Since $\left|X_{w}\right|=64$ and $4 X_{w}=0$ and there are 48 elements in $X_{w}$ of order four, $X_{w}$ must have type $(4,4,2,2)$, with an automorphism group of order

$$
\left|\operatorname{Aut}\left(X_{w}\right)\right|=2^{14} \cdot 3^{2} \text {. }
$$

It follows from the above count that $\operatorname{im} \varrho_{w}$ is transitive on the $\operatorname{Aut}(X)$-orbits in $X_{w}$.

We can write $W_{1}=W\left(D_{3}\right) \times W\left(D_{5}\right)$ and correspondingly $\hat{T}=\hat{T}_{3} \times_{Z} \hat{T}_{5}$, where $\hat{T}_{n}$ is a maximal torus in $\operatorname{Spin}_{n}$ and $Z \simeq \mu_{4}$ is the diagonally embedded center of each factor. Let $s \in \hat{T}_{5}$ have Kac coordinates equal to 1 on a branch node, and 
zero on all other nodes. We can take $w$ to be a Coxeter element in the centralizer $C_{W_{1}}(s) \simeq W\left(D_{3}\right) \times\left[W\left(D_{3}\right) \times W\left(D_{2}\right)\right]$. Write $w=a b c$ accordingly. Then

$$
\operatorname{ker} \varrho_{w} \subset C_{W_{1}}(s, w)=\langle a\rangle \times\langle b\rangle \times\langle c\rangle .
$$

Applying Lemma 2.4 to elements in $C_{W_{1}}(s, w)$ shows that $\operatorname{ker} \varrho_{w}=\langle w\rangle$, so that $\left|\operatorname{im} \varrho_{w}\right|=2^{9} \cdot 3^{2}$.

We show that $\varrho_{w}$ is surjective by computing the order of the group $\operatorname{Sp}\left(X_{w}\right)$ of automorphisms of $X_{w} \simeq(\mathbb{Z} / 4 \mathbb{Z})^{2} \oplus(\mathbb{Z} / 2 \mathbb{Z})^{2}$ preserving the alternating form $\langle,\rangle_{w}$. Since the class of $w$ is determined by $\operatorname{det}(t-w)$, we can check that $w=v^{3}$, where $v$ belongs to the class $A_{3} D_{5}\left(a_{1}\right)$. The $v$-invariants in $X_{w} \simeq \hat{T}^{w}$ are a submodule $U \simeq \hat{T}^{v} \simeq(\mathbb{Z} / 4 \mathbb{Z})^{2}$. Choose an element $y \in X_{w}$ of order two such that $\langle y, U\rangle_{w}=0$. Since $v$ has order three on $X_{w}$, we have $y+v y+v^{2} y=0$ and $\left\{0, y, v y, v^{2} y\right\}$ is a subspace $Y \simeq(\mathbb{Z} / 2 \mathbb{Z})^{2}$ giving an orthogonal decomposition

$$
X_{w}=U \oplus Y .
$$

Applying the argument of 5.9 to $U$, we can choose bases $\left\{u_{1}, u_{2}\right\},\left\{y_{1}, y_{2}\right\}$ of $U$ and $Y$ such that

$$
\left\langle u_{1}, u_{2}\right\rangle=\left\langle y_{1}, y_{2}\right\rangle=1, \quad\left\langle u_{1}, u_{1}\right\rangle=\left\langle y_{1}, y_{1}\right\rangle=0 .
$$

We compute the stabilizer of $u_{1}$ in $S p\left(X_{w}\right)$ : Suppose

$$
g=\left[\begin{array}{llll}
1 & a & x & z \\
0 & b & y & t \\
0 & c & p & r \\
0 & d & q & s
\end{array}\right] \in S p\left(X_{w}\right) .
$$

Here, $a, b \in \mathbb{Z} / 4 \mathbb{Z}, c, d, p, q, r, s \in \mathbb{Z} / 2 \mathbb{Z}$, and $x, y, z, t \in 2 \mathbb{Z} / 4 \mathbb{Z}$. We have

$$
\begin{aligned}
& 0=\left\langle u_{1}, v_{1}\right\rangle_{w}=\left\langle u_{1}, g v_{1}\right\rangle_{w}=y, \\
& 0=\left\langle u_{1}, v_{2}\right\rangle_{w}=\left\langle u_{1}, g v_{2}\right\rangle_{w}=t, \\
& 1=\left\langle u_{1}, u_{2}\right\rangle_{w}=\left\langle u_{1}, g u_{2}\right\rangle_{w}=b, \\
& 0=\left\langle u_{2}, v_{1}\right\rangle_{w} \Rightarrow x=c q+d p, \\
& 0=\left\langle u_{2}, v_{2}\right\rangle_{w} \Rightarrow z=c s+d r, \\
& 1=\left\langle v_{1}, v_{2}\right\rangle_{w}=p s+q r .
\end{aligned}
$$

Therefore,

$$
g=\left[\begin{array}{llll}
1 & a & x & z \\
0 & 1 & 0 & 0 \\
0 & c & p & r \\
0 & d & q & s
\end{array}\right]
$$

where $x=c q+d p$ and $z=c s+d r$. We find exactly $2^{5} \cdot 3$ choices for $g$. Since $C(w)$ is already transitive on elements of order four in $X_{w}$, then $S p\left(X_{w}\right)$ is also, and we have

$$
\left|S p\left(X_{w}\right)\right|=48 \cdot 2^{5} \cdot 3=2^{9} \cdot 3^{2}=\left|\operatorname{im} \varrho_{w}\right| .
$$

Hence $\varrho_{w}$ is surjective, as claimed. 


\section{MAXIMAL TORI IN QUASI-SPLIT GROUPS}

This section and the next contain applications of our study of coinvariant representations to the classification of maximal tori in $p$-adic groups and associated supercuspidal $L$-packets. We begin in greater generality, with the rough classification of maximal tori in quasi-split groups over any perfect field $k$. In the spirit of the Langlands correspondence, the rational classes of maximal tori will be partitioned into "stable classes", in which the rational classes correspond to orbits of a certain rational Weyl group on a Galois cohomology group. When we specialize $k$ to be a $p$-adic field, this becomes a coinvariant representation, or subquotient thereof, twisted by a cocycle which we compute in many cases.

6.1. Rational and stable conjugacy. For basic results in Galois cohomology we refer [32]. Let $k$ be a perfect field and let $\Gamma=\operatorname{Gal}(\bar{k} / k)$ be the absolute Galois group of $k$. For any algebraic $k$-group $L$, let $H^{1}(k, L)=H^{1}(\bar{k} / k, L)$ denote the first Galois cohomology set of $L$. If $M$ is a subgroup of $L$ defined over $k$, let $\operatorname{ker}^{1}(M, L)$ denote the kernel of the map $H^{1}(k, M) \rightarrow H^{1}(k, L)$ induced by the inclusion $M \hookrightarrow L$.

Let $G$ be a connected semisimple quasi-split algebraic group defined over $k$. By maximal torus in $G$ we mean a subgroup of $G$ which is a maximal torus and is defined over $k$. We consider two notions of conjugacy: Two maximal tori $S, S^{\prime}$ in $G$ are rationally congugate if they are conjugate by an element of $G(k)$, and they are stably conjugate if their groups $S(k)$ and $S^{\prime}(k)$ of rational points are conjugate by an element of $G=G(\bar{k})$. Each stable class is partitioned into rational classes.

Let $A$ be a maximal $k$-split torus in $G$ and let $T=C_{G}(A)$ be the centralizer of $A$. Then $T$ is a maximal torus in $G$, since $G$ is quasi-split. The Weyl group $W$ is the quotient $N / T$, where $N$ is the normalizer of $T$. Let $\pi: H^{1}(k, N) \rightarrow H^{1}(k, W)$ be the map induced by the projection $N \rightarrow W$ and let $\pi_{1}: \operatorname{ker}^{1}(N, G) \rightarrow H^{1}(k, W)$ be the restriction of $\pi$ to $\operatorname{ker}^{1}(N, G)$.

Proposition 6.1. The stable classes of maximal tori in $G$ are in bijection with $H^{1}(k, W)$. The set of rational classes of maximal tori in the stable class corresponding to a class $x \in H^{1}(k, W)$ is in bijection with the fiber $\pi_{1}^{-1}(x) \subset \operatorname{ker}^{1}(N, G)$.

When $k$ is finite, we have $H^{1}(k, G)=1$ and $\pi_{1}=\pi$ is actually a bijection, as follows from the Lang-Steinberg theorem. Each stable class consists of a single rational class and these classes are in bijection with $H^{1}(k, W)$, as is well known (cf. [5]). When $k$ is $p$-adic, a version of Proposition 6.1 was proved for unramified tori by DeBacker [12].

From now on, we assume that $k$ is infinite and we identify algebraic $k$-groups with their groups of $k$-rational points. The proof of Proposition 6.1 will occupy the rest of this section.

Lemma 6.2. The set of rational conjugacy classes of maximal tori in $G$ is in bijection with $\operatorname{ker}^{1}(N, G)$.

Proof. This is a special case of a basic principal in Galois cohomology. Indeed, if $S$ is a maximal torus in $G$ then $S=g T g^{-1}$ for some $g$ in $G$, and since $S$ is defined over $k$ we have $g^{-1} \gamma(g) \in N$ for all $\gamma \in \Gamma$. Sending the rational class of $S$ to the class of the cocycle $\gamma \mapsto g^{-1} \gamma(g)$ gives the asserted bijection.

Next, by a result of Raghunathan, we know that each fiber of $\pi_{1}$ is nonempty.

Lemma 6.3. The map $\pi_{1}: \operatorname{ker}^{1}(N, G) \rightarrow H^{1}(k, W)$ is surjective. 
Proof. See [27], which requires $G$ to be quasi-split, as we have assumed.

The proof of Proposition 6.1 is completed by the next result:

Lemma 6.4. Let $S, S^{\prime}$ be maximal tori in $G$ whose rational classes correspond to $c, c^{\prime} \in \operatorname{ker}^{1}(N, G)$ as in Lemma 6.2. Then $S$ and $S^{\prime}$ are stably conjugate if and only if $\pi_{1}(c)=\pi_{1}\left(c^{\prime}\right)$.

Proof. Write $S={ }^{h} T, S^{\prime}={ }^{\ell} T$, so that

$$
\xi_{\gamma}=h^{-1} \gamma(h) \in c \quad \text { and } \quad \eta_{\gamma}=\ell^{-1} \gamma(\ell) \in c^{\prime} .
$$

Suppose that $\operatorname{Ad}(g)[S(k)]=S^{\prime}(k)$ for some $g \in G$. Since $k$ is infinite, $S(k)$ is Zariski-dense in $S$ and likewise for $S^{\prime}$. In particular, it follows that ${ }^{g h} T={ }^{\ell} T$, so we get an element

$$
n:=\ell^{-1} g h \in N .
$$

I claim that $n \xi_{\gamma} \gamma(n)^{-1}$ and $\eta_{\gamma}$ have the same image in $W$.

Choose an element $s \in S^{\prime}(k)$ whose centralizer is $S^{\prime}$. We have $s^{g} \in S(k)$, so for each $\gamma \in \Gamma$ the element $g \gamma(g)^{-1}$ centralizes $s$, hence belongs to $S^{\prime}$. Now,

$$
n \xi_{\gamma} \gamma(n)^{-1}=n h^{-1} \gamma\left(h n^{-1}\right)=\ell^{-1} g \gamma\left(g^{-1} \ell\right),
$$

so it must be shown that

$$
\ell^{-1} g \gamma\left(g^{-1} \ell\right) \in \ell^{-1} \gamma(\ell) T \quad \text { for all } \quad \gamma \in \Gamma .
$$

But this holds because

$$
\gamma(\ell)^{-1} g \gamma\left(g^{-1} \ell\right)=\eta_{\gamma}^{-1} \ell^{-1} g \gamma(g)^{-1} \ell \eta_{\gamma} \in \operatorname{Ad}\left(\eta_{\gamma}^{-1}\right) \operatorname{Ad}\left(\ell^{-1}\right) S^{\prime}=\operatorname{Ad}\left(\eta_{\gamma}^{-1}\right) T=T .
$$

For the converse, suppose $\xi_{\gamma}$ and $\eta_{\gamma}$ are as in (30) and that $t_{\gamma} \xi_{\gamma}=\eta_{\gamma}$ for some cocycle $t_{\gamma} \in T$. Let $g=\ell h^{-1}$. Then

$$
\gamma(g)=\ell t_{\gamma} h^{-1} .
$$

For any $s \in S(k)$ we have $\operatorname{Ad}\left(h^{-1}\right) s \in T$, so

$$
\gamma\left(g s g^{-1}\right)=\operatorname{Ad}\left(\ell t_{\gamma} h^{-1}\right) s=\operatorname{Ad}\left(\ell h^{-1}\right) s=g s g^{-1} .
$$

This shows that $\operatorname{Ad}(g)$ maps $S(k)$ to $S^{\prime}(k)$, and completes the proof of Proposition 6.1.

6.2. The fibers of $\pi_{1}$. To determine the rational classes in a stable class of maximal tori, we study the fibers of $\pi_{1}$. This requires the notion of twisting in Galois cohomology. If $L$ is an algebraic $k$-group and $\xi: \Gamma \rightarrow \operatorname{Aut}(L)$ is a Galois cocycle, then $L_{\xi}$ denotes the $k$-group twisted by $\xi$ : we have $L_{\xi}=L$ as sets, with new $\Gamma$ action given by $\gamma_{\xi}(\ell)=\xi_{\gamma} \cdot \gamma(\ell)$, where $\gamma(\ell)$ is the original action of $\Gamma$ on $L$ and $\xi_{\gamma}$. is the action of $\operatorname{Aut}(L)$ on $L$. If $\xi$ takes values in $L$ instead of $\operatorname{Aut}(L)$, the twisting is understood with respect to the cocycle $\operatorname{Ad}(\xi): \Gamma \rightarrow \operatorname{Aut}(L)$ given by $\operatorname{Ad}(\xi)_{\gamma}(\ell)=\xi_{\gamma} \ell \xi_{\gamma}^{-1}$.

Fix a cocycle $\xi: \Gamma \rightarrow W$ and let $T_{\xi}$ be the twist of $T$ via the natural map $W \rightarrow \operatorname{Aut}(T)$. By Lemma 6.3 there exists $g \in G$ such that the cocycle $\dot{\xi}_{\gamma}:=g^{-1} \gamma(g)$ takes values in $N$ and whose projection to $W$ lies in the class of $\xi$. One checks that the conjugation map $\operatorname{Ad}(g): T_{\xi} \rightarrow g T g^{-1}$ is $k$-rational. Thus, the twisted torus $T_{\xi}$ embeds as a maximal torus in $G$ for any cocycle $\xi: \Gamma \rightarrow W$. 
We also have the twisted groups $N_{\dot{\xi}}$ and $G_{\dot{\xi}}$. The latter is $k$-isomorphic to $G$ via the $\operatorname{map} \operatorname{Ad}(g): G_{\dot{\xi}} \rightarrow G$ and we have a commutative square, whose horizontal maps are induced by inclusion and whose vertical maps are bijections:

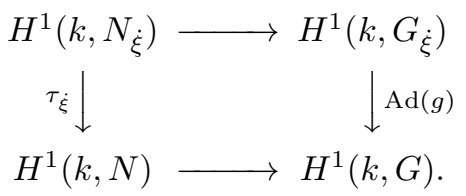

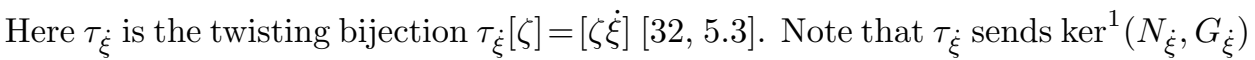
to $\operatorname{ker}^{1}(N, G)$.

The exact sequence of $k$-groups

$$
1 \longrightarrow T_{\xi} \longrightarrow N_{\dot{\xi}} \longrightarrow W_{\xi} \longrightarrow 1
$$

gives an exact sequence of pointed sets [32, 5.1(2)] in the top row of the following diagram:

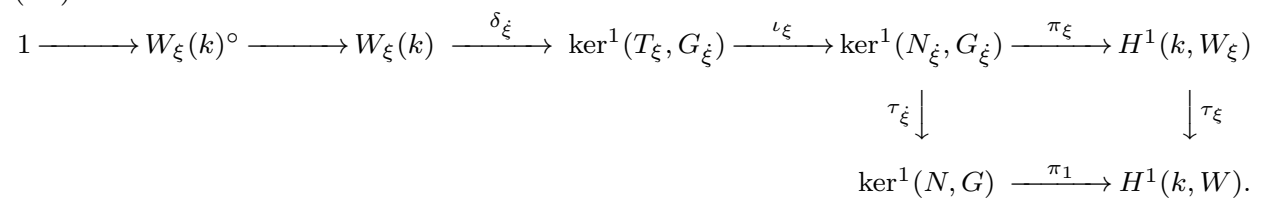

Here $W_{\xi}(k)=\left\{w \in W: \xi_{\gamma} \gamma(w) \xi_{\gamma}^{-1}=w\right\}$ is the group of $k$-rational points in the twisted group $W_{\xi}$ and $W_{\xi}(k)^{\circ}=N_{\dot{\xi}}(k) / T_{\xi}(k)$ consists of the elements in $W_{\xi}(k)$ having a $k$-rational representative in $N_{\dot{\xi}}$. The maps $\iota_{\xi}, \pi_{\xi}, \pi_{1}$ are induced by the maps on underlying groups. The map $\tau_{\xi}$ is the projection of the previous $\tau_{\dot{\xi}}$, and is given by $\tau_{\xi}[\eta]=[\eta \xi]$. Finally, $\delta_{\dot{\xi}}$ is the connecting map: For $w \in W_{\xi}(k), \delta_{\dot{\xi}}(w)$ is the class of cocycles $\Gamma \rightarrow T_{\xi}$ of the form

$$
\gamma \mapsto \dot{w} \gamma_{\dot{\xi}}(\dot{w})^{-1}=\dot{w} \dot{\xi}_{\gamma} \gamma(\dot{w})^{-1} \dot{\xi}_{\gamma}^{-1}
$$

where $\dot{w} \in N$ is a lift of $w$.

The group $W_{\xi}(k)$ acts on $H^{1}\left(k, T_{\xi}\right)$ via the rule

$$
(w * \zeta)_{\gamma}=\dot{w} \zeta_{\gamma} \gamma_{\xi}(\dot{w})^{-1},
$$

where $\dot{w}$ is a lift of $w$ in $N$ and $\zeta$ is a cocycle in $T_{\xi}$. This is an affine action of $W_{\xi}(k)$ on the abelian group $H^{1}\left(k, T_{\xi}\right)$. Indeed, the connecting map $\delta_{\dot{\xi}}: W_{\xi}(k) \rightarrow$ $H^{1}\left(k, T_{\xi}\right)$ is a cocycle on $W_{\xi}(k)$ and we have

$$
w * \zeta=(w \cdot \zeta) \delta_{\dot{\xi}}(w)
$$

where $w \cdot \zeta$ is the linear action of $W_{\xi}(k)$ on $H^{1}\left(k, T_{\xi}\right)$ induced by the natural action of $W$ on $T$ (cf. [32, I.5.6]). Suppose the class of $\zeta$ belongs to $\operatorname{ker}^{1}\left(T_{\xi}, G_{\dot{\xi}}\right)$, so that $\zeta_{\gamma}=h^{-1} \gamma_{\dot{\xi}}(h)$ for some $h \in G$. Then $(w * \zeta)_{\gamma}=\dot{w} h^{-1} \gamma_{\dot{\xi}}\left(h \dot{w}^{-1}\right)$. Hence the affine action of $W_{\xi}(k)$ on $H^{1}\left(k, T_{\xi}\right)$ preserves $\operatorname{ker}^{1}\left(T_{\xi}, G_{\dot{\xi}}\right)$.

By Proposition 6.1, the class $x \in H^{1}(k, W)$ of $\xi$ determines a stable class $\mathcal{T}_{x}$ of maximal tori in $G$ and the rational classes in $\mathcal{T}_{x}$ are in bijection with the fiber $\pi_{1}^{-1}(x)$. The next result makes this more precise.

Proposition 6.5. The rational classes in $\mathcal{T}_{x}$ are in bijection with the orbits of $W_{\xi}(k)$ on $\operatorname{ker}^{1}\left(T_{\xi}, G_{\dot{\xi}}\right)$ under the affine action. 
Proof. This is a special case of [32, I.5.5]. Under the twisting bijection $\tau_{\dot{\xi}}$, we have

$$
\pi_{1}^{-1}(x)=\tau_{\dot{\xi}}\left(\operatorname{ker} \pi_{\xi}\right)=\tau_{\dot{\xi}}\left(\operatorname{im} \iota_{\xi}\right) .
$$

It follows that the set of rational classes in $\mathcal{T}_{x}$ are in bijection with the set of fibers of $\iota_{\xi}$. From the definition of $H^{1}\left(k, N_{\dot{\xi}}\right)$, the fibers of $\iota_{\xi}$ are the orbits of $W_{\xi}(k)$ in $H^{1}\left(k, T_{\xi}\right)$ under the affine action.

The affine action depends on the choice of lift $\dot{\xi}$. A different lift $\ddot{\xi}$ of $\xi$ gives an affine action which is conjugate to the original one by a translation of the group $H^{1}\left(k, T_{\xi}\right)$. Equivalently, the cocycles $\delta_{\dot{\xi}}, \delta_{\ddot{\xi}}$ are cohomologous as cocycles $W_{\xi}(k) \rightarrow$ $H^{1}\left(k, T_{\xi}\right)$. Thus, from the cocycle $\xi: \Gamma \rightarrow W$ we get a class

$$
\Delta_{\xi}=\left[\delta_{\dot{\xi}}\right] \in H^{1}\left(W_{\xi}(k), H^{1}\left(k, T_{\xi}\right)\right)
$$

which is independent of the choice of lift $\dot{\xi}$. This class $\Delta_{\xi}$ vanishes exactly when $\dot{\xi}$ can be chosen to make $\delta_{\dot{\xi}} \equiv 1$, in which case the affine and linear actions of $W_{\xi}(k)$ on $H^{1}\left(k, T_{\xi}\right)$ coincide. In section 6.6 we will compute the class $\Delta_{\xi}$ when $k$ is a $p$-adic field, for various kinds of cocycles $\xi: \Gamma \rightarrow W$.

6.3. Weyl groups. A maximal torus $S$ in $G$ has several Weyl groups: The absolute Weyl group of $S$ is the quotient $W_{S}=N_{S} / S$, where $N_{S}$ is the normalizer of $S$ in $G$. The big rational Weyl group of $S$ is the group $W_{S}(k)=W_{S}^{\Gamma}$ of $k$-rational points in $W_{S}$. Finally, the small rational Weyl group is the subgroup $W_{S}(k)^{\circ} \subset W_{S}(k)$ consisting of elements in $W_{S}(k)$ which have a representative in $N_{S}(k)=N_{S}^{\Gamma}$.

Suppose $S$ corresponds to the class of the cocycle $\dot{\xi}$ in $N$, and let $\xi$ be the projection of $\dot{\xi}$ to $W$. If $\dot{\xi}_{\gamma}=g^{-1} \gamma(g)$, the map $\operatorname{Ad}(g)$ gives an isomorphism $W_{\xi} \rightarrow W_{S}$ which is defined over $k$. Hence $\operatorname{Ad}(g)$ restricts to an isomorphism

$$
W_{\xi}(k) \stackrel{\sim}{\longrightarrow} W_{S}(k) .
$$

In particular, the isomorphism type of $W_{S}(k)$ depends only on the stable class of $S$ corresponding to the class of $\xi$ in $H^{1}(k, W)$.

We have seen that the rational classes of maximal tori in the stable class of $S$ are in bijection, via the twisting bijection $\tau_{\dot{\xi}}$, with the image of the map

$$
\iota_{\xi}: \operatorname{ker}^{1}\left(T_{\xi}, G_{\dot{\xi}}\right) \rightarrow \operatorname{ker}^{1}\left(N_{\dot{\xi}}, G_{\dot{\xi}}\right)
$$

induced by the inclusion $T \hookrightarrow N$. Explicitly, a cocycle $\zeta: \Gamma \rightarrow T_{\xi}$ corresponds to the maximal torus $S_{\zeta}:=h T h^{-1}$, where $h^{-1} \gamma(h)=\zeta_{\gamma} \dot{\xi}_{\gamma}$. Let $W_{\xi}(k, \zeta)$ denote the stabilizer in $W_{\xi}(k)$, under the affine action, of the class of $\zeta$ in $\operatorname{ker}^{1}\left(T_{\xi}, G_{\dot{\xi}}\right)$.

Proposition 6.6. The map $\operatorname{Ad}(h)$ restricts to an isomorphism $W_{\xi}(k, \zeta)$ $\stackrel{\sim}{\longrightarrow} W_{S_{\zeta}}(k)^{\circ}$. Thus, the small rational Weyl group of $S$ is isomorphic to a pointstabilizer in $W_{\xi}(k)$ on the orbit in $\operatorname{ker}^{1}\left(T_{\xi}, G_{\dot{\xi}}\right)$ corresponding to $S$ as in Proposition 6.5.

Proof. It is straightforward to check that any $w \in W_{\xi}(k, \zeta)$ has a lift $\dot{w} \in N$ fixing the cocycle $\zeta$ itself, that is, we may choose $\dot{w}$ so that $\dot{w} \zeta_{\gamma} \gamma_{\dot{\xi}}(\dot{w})^{-1}=\zeta_{\gamma}$ for all $\gamma \in \Gamma$. It then follows that $\gamma\left(h \dot{w} h^{-1}\right)=h \dot{w} h^{-1}$ for all $\gamma \in \Gamma$. Therefore $h \dot{w} h^{-1} \in N_{S_{\zeta}}(k)$ as desired. The argument is reversible. 
6.4. Example: $\mathbf{S L}_{\mathbf{2}}$. The class $\Delta_{\xi} \in H^{1}\left(W_{\xi}(k), H^{1}\left(k, T_{\xi}\right)\right)$ can be nontrivial. Let $G=S L_{2}$ and let $W$ be the Weyl group of the diagonal torus in $T \subset G$. A nontrivial cocycle $\xi: \Gamma \rightarrow W$ is a homomorphism factoring through an isomorphism $\operatorname{Gal}(E / k) \rightarrow W$, where $E / k$ is a quadratic extension, with absolute Galois group $\Gamma_{E}=\operatorname{ker} \xi$. The twisted torus $T_{\xi}$ is the one-dimensional unitary group $U_{1}$ of $E / k$ and $W_{\xi}(k)=W$. By Hilbert's Theorem 90, any cocycle $c: \Gamma \rightarrow T_{\xi}$ may be adjusted by a coboundary so as to take just two values:

$$
c_{\gamma}= \begin{cases}1 & \text { if } \quad \gamma \in \Gamma_{E}, \\ x & \text { if } \quad \gamma \in \Gamma-\Gamma_{E},\end{cases}
$$

for some $x \in T(k)=k^{\times}$. This leads to the isomorphism

$$
H^{1}\left(k, T_{\xi}\right) \simeq k^{\times} / N E^{\times},
$$

where $N E^{\times}$is the norm group of $E$. Since $x^{-1} \equiv x \bmod N E^{\times}$, it follows that the linear action of $W$ on $H^{1}\left(k, T_{\xi}\right)$ is trivial. Hence the cocycle $\delta_{\dot{\xi}}$ is independent of the choice of lift $\dot{\xi}$, and is simply a group homomorphism

$$
\delta_{\xi}: W \rightarrow k^{\times} / N E^{\times}
$$

which is determined by the image $\delta_{\xi}(w)$ of the nontrivial element $w \in W$.

Proposition 6.7. $\delta_{\xi}(w)$ is the class of -1 in $k^{\times} / N E^{\times}$.

Proof. If char $k=2$, then the projection $N \rightarrow W$ splits, which implies that $\delta_{\xi}$ is trivial, just as -1 is trivial in $k^{\times} / N E^{\times}$. Assume that char $k \neq 2$ and write $E=k(\epsilon)$, where $\epsilon=\sqrt{e}$ and $e$ is a nonsquare in $k^{\times}$.

There is a torus $S$ in the stable class $\mathcal{T}_{\xi}$ with rational points

$$
S(k)=\left\{\left[\begin{array}{cc}
a & e b \\
b & a
\end{array}\right]: a, b \in k^{\times}, a^{2}-e b^{2}=1\right\} .
$$

We have $S=g T g^{-1}$, where

$$
g=\left[\begin{array}{cc}
1 & -\epsilon / 2 \\
1 / \epsilon & 1 / 2
\end{array}\right]
$$

and $S$ determines the lifted cocycle given (for $\gamma \in \Gamma-\Gamma_{E}$ ) by

$$
\dot{\xi}_{\gamma}=g^{-1} \gamma(g)=\left[\begin{array}{cc}
0 & -2 / \epsilon \\
\epsilon / 2 & 0
\end{array}\right] \in N(E) .
$$

Choosing $\dot{w}=\left[\begin{array}{rr}0 & -1 \\ 1 & 0\end{array}\right]$, the class of $\delta_{\xi}(w)$ in $H^{1}\left(k, T_{\xi}\right)$ is represented by the cocycle $\delta_{\dot{\xi}}(\dot{w})$ given by

$$
\delta_{\dot{\xi}}(\dot{w})_{\gamma}=\dot{w} \dot{\xi}_{\gamma} \dot{w}^{-1} \dot{\xi}_{\gamma}^{-1}=\left[\begin{array}{cc}
e / 4 & 0 \\
0 & 4 / e
\end{array}\right] .
$$

Since $e / 4$ and -1 have the same class in $k^{\times} / N E^{\times}$, the proposition is proved.

An alternative proof runs as follows: Viewing $E$ as a two-dimensional $k$-vector space, the normalizer of $S$ in $G L_{2}(k)$ is $E^{\times} \rtimes\langle\sigma\rangle$, where $\sigma$ generates $\operatorname{Gal}(E / k)$. Since $\operatorname{det} \sigma=-1$, there is an element of $N_{S}(k)$ outside $S(k)$ exactly when there is an element of $E^{\times}$of norm equal to -1 . Since $|W|=2$, the proposition now follows from Proposition 6.6. 
Corollary 6.8. The stable class $\mathcal{T}_{\xi}$ consists of a single rational class of tori precisely when -1 is not a norm from $E^{\times}$. Otherwise, $\mathcal{T}_{\xi}$ is a union of two rational classes of tori.

6.5. $p$-adic fields. From now on, $k$ is a finite extension of $\mathbb{Q}_{p}$ for some prime $p$, and $\Gamma=\operatorname{Gal}(\bar{k} / k)$ as before. Let $S$ be a torus over $k$ with cocharacter group $X=X_{*}(S)$. Then $\Gamma$ acts on $X$ via a continuous homomorphism $\varphi: \Gamma \rightarrow \operatorname{Aut}(X)$. Let $X_{\varphi}$ denote the coinvariants of $\Gamma$ acting on $X$ via $\varphi$, and let $\left[X_{\varphi}\right]_{\text {tor }}$ be the torsion subgroup of $X_{\varphi}$. Tate-Nakayama duality gives a natural isomorphism

$$
\left[X_{\varphi}\right]_{\text {tor }} \stackrel{\sim}{\longrightarrow} H^{1}(k, S) .
$$

If the image $\varphi(\Gamma)=\langle w\rangle$ is cyclic, generated by $w \in \operatorname{Aut}(X)$, then $X_{\varphi}=X_{w}$ as considered earlier.

Keeping the notation of section 6.1, it is suggestive to reformulate the classification of maximal tori in $G$ to emphasize the analogy with the local Langlands conjecture for representations of $G(k)$. Let $R \subset X^{*}(T)$ be the set of roots of $T$ in $G$. There is a base $B \subset R$ preserved by $\Gamma$, since $G$ is quasi-split, and the $k$-structure on $G$ is given (up to isomorphism) by a continuous homomorphism

$$
\psi_{G}: \Gamma \longrightarrow \operatorname{Aut}(R, B)
$$

from $\Gamma$ into the group of automorphisms of $R$ preserving $B$. The semidirect product

$$
{ }^{L} W:=W \rtimes \operatorname{Aut}(R, B)
$$

acts faithully on the cocharacter group $X=X_{*}(T)$. We define a Langlands parameter for $W$ to be a continuous homomorphism

$$
\varphi: \Gamma \rightarrow{ }^{L} W
$$

whose projection onto $\operatorname{Aut}(R, B)$ is the map $\psi_{G}$. For each $\gamma \in \Gamma$, we have $\varphi(\gamma)=$ $\xi_{\gamma} \rtimes \psi_{G}(\gamma)$, where $\xi: \Gamma \rightarrow W$ is a cocycle. Two parameters are $W$-conjugate iff their cocycles are cohomologous. From (35) we may identify $H^{1}\left(k, T_{\xi}\right)=\left[X_{\varphi}\right]_{\text {tor }}$. The isomorphism (35) was generalized by Kottwitz [22] to an isomorphism

$$
(X / \mathbb{Z} \check{R})_{\psi_{G}} \stackrel{\sim}{\longrightarrow} H^{1}(k, G)
$$

where $\mathbb{Z} \check{R}$ is the coroot lattice and the left side of $(\underline{36})$ is the coinvariants of $\Gamma$ acting on $X / \mathbb{Z} \check{R}$ via $\psi_{G}$. Let $\left[X_{\varphi}\right]_{\text {tor }}^{1}$ be the kernel of the natural projection $\left[X_{\varphi}\right]_{\text {tor }} \rightarrow$ $(X / \mathbb{Z} \check{R})_{\psi_{G}}$. It follows from [23, Thm. 1.2] that if $T_{\varphi}$ embeds as a torus $S$ in $G$, then (35) restricts to an isomorphism

$$
\left[X_{\varphi}\right]_{\text {tor }}^{1} \stackrel{\sim}{\longrightarrow} \operatorname{ker}^{1}(S, G)
$$

As above, let $\xi: \Gamma \rightarrow W$ be the cocycle obtained by projecting $\varphi$ to $W$. The big rational Weyl group $W_{\xi}(k)$ coincides with the centralizer $C_{W}(\varphi)$ in $W$ of the image of $\varphi(\Gamma)$, and the linear action of $W_{\xi}(k)$ on $H^{1}\left(k, T_{\xi}\right)$ is identified with the coinvariant representation of $C_{W}(\varphi)$ on $\left[X_{\varphi}\right]_{\text {tor }}$. This action preserves the subspace $\left[X_{\varphi}\right]_{\text {tor }}^{1}$. Let $\Delta_{\varphi}:=\Delta_{\xi} \in H^{1}\left(C_{W}(\varphi),\left[X_{\varphi}\right]_{\text {tor }}^{1}\right)$ be the cohomology class associated to $\xi$ as in (33). Propositions 6.1, 6.5 and 6.6 may be summarized in this new language as follows.

Theorem 6.9. The $W$-conjugacy classes of Langlands parameters $\varphi: \Gamma \rightarrow{ }^{L} W$ are in bijection with the stable classes of maximal tori in $G$. Denoting this correspondence by $\varphi \mapsto \mathcal{T}_{\varphi}$, we have:

1. $W_{S}(k) \simeq C_{W}(\varphi)$ and $\operatorname{ker}^{1}(S, G) \simeq\left[X_{\varphi}\right]_{\text {tor }}^{1}$, for any $S \in \mathcal{T}_{\varphi}$. 
2. The rational classes in $\mathcal{T}_{\varphi}$ are in bijection with the orbits of $C_{W}(\varphi)$ in $\left[X_{\varphi}\right]_{\text {tor }}^{1}$ under the affine action obtained by twisting the coinvariant representation by a cocycle belonging to the class of $\Delta_{\varphi}$.

3. If $S \in \mathcal{T}_{\varphi}$ has rational class corresponding to the orbit of $\lambda \in\left[X_{\varphi}\right]_{\text {tor }}^{1}$ under the affine action in 2 , then the small rational Weyl group $W_{S}(k)^{\circ}$ is isomorphic to the stabilizer of $\lambda$ in $C_{W}(\varphi)$.

Thus, a stable class of maximal tori is analogous to a stable $L$-packet of representations of $G(k)$. In fact, this is more than an analogy: One can regard the $L$-packets of supercuspidal representations constructed in [13, 21, [30] as "induced" from $L$ packets of tori as in Theorem 6.9, see section 6.8 below.

6.6. Rational lifting. We have seen that the class $\Delta_{\varphi}$ in Theorem 6.9 can be nontrivial, even for $S L_{2}$ (cf. 6.7). However, $\Delta_{\varphi}$ does vanish in many cases. For example, suppose $G$ is a split group isomorphic to $S L_{2 n+1}, S O_{n}$ or $G_{2}$. In these cases there is a subgroup of $N(k)$ projecting isomorphically onto $W$, so any parameter(=cocycle) $\varphi: \Gamma \rightarrow W$ has an obvious lift $\dot{\varphi}: \Gamma \rightarrow N(k)$ for which $\delta_{\dot{\varphi}}$ is trivial, so that $\Delta_{\varphi}=1$.

If there is no subgroup of $N(k)$ projecting isomorphically onto $W$, one can often lift enough of $W$ to kill $\Delta_{\varphi}$. This section describes two such cases.

6.6.1. Unramified tori. Let the residue field $\mathfrak{f}$ of our $p$-adic field $k$ have cardinality $q$ and let $\overline{\mathfrak{f}}$ be an algebraic closure of $\mathfrak{f}$. The kernel of the natural action of $\Gamma$ on $\overline{\mathfrak{f}}$ is the inertia subgroup $\mathcal{I} \triangleleft \Gamma$, whose fixed field in $\bar{k}$ is a maximal unramified extension $K / k$, and

$$
\Gamma / \mathcal{I} \simeq \operatorname{Gal}(K / k) \simeq \operatorname{Gal}(\overline{\mathfrak{f}} / \mathfrak{f}) .
$$

We fix an element $\mathrm{F} \in \Gamma$ whose inverse projects to the $q$-power map in $\operatorname{Gal}(\overline{\mathfrak{f}} / \mathfrak{f})$. A cocycle or homomorphism from $\Gamma$ into another group is called unramified if it is trivial on $\mathcal{I}$. Such a map is completely determined by its value on F. A $k$-torus $S$ is called unramified if the $\mathcal{I}$ acts trivially on $X_{*}(S)$.

Assume that $G$ splits over $K$. Equivalently, assume that our maximally split torus $T$ is unramified. A twisted torus $T_{\xi}$ is unramified precisely when the cocycle $\xi: \Gamma \rightarrow W$ is unramified, in which case $\xi$ is determined by an element $w:=\xi(\mathrm{F}) \in$ $W$.

Proposition 6.10. If $\varphi: \Gamma \rightarrow W$ is an unramified parameter, then $\Delta_{\varphi}$ is trivial.

Proof. Since $G$ is $K$-split and $k$-quasi-split, there is an F-stable hyperspecial vertex $o$ in the apartment $\mathcal{A}(K)$ of $T(K)$ in the Bruhat-Tits building of $G(K)$. The group $N(K)$ acts on $\mathcal{A}(K)$ and we let $N(K)_{o}$ denote the stabilizer of $o$ in $N(K)$. The subgroup $T(K)_{0}=T \cap N(K)_{o}$ is a pro-algebraic $\mathfrak{f}$-group acting trivially on $\mathcal{A}(K)$. Let $\xi$ be the projection of $\varphi$ to $W$ and let $w=\xi(\mathrm{F})$. Any lift $\dot{w} \in N(K)_{o}$ of $w$ gives a lift of $\xi$ to an unramified cocycle $\dot{\xi}: \Gamma \rightarrow \dot{W}$ such that $\dot{\xi}(\mathrm{F})=\dot{w}$. Let $x \in W_{\xi}(k)$ and choose any lift $\dot{x} \in N(K)$ of $x$, so that $\delta_{\dot{\xi}}(\dot{x})=\dot{x} \dot{w} \mathrm{~F}(\dot{x})^{-1} \dot{w}^{-1}$. Applying the Lang-Steinberg theorem to the pro-algebraic f-group $T(K)_{0}$ twisted by $w$, we get an element $t \in T(K)_{0}$ for which $t^{-1} \dot{w} \mathrm{~F}(t) \dot{w}^{-1}=\delta_{\dot{\xi}}(\dot{x})$. Then we have $\delta_{\dot{\xi}}(\dot{x} t)=1$. It follows that $\delta_{\dot{\xi}}: \Gamma \rightarrow H^{1}\left(k, T_{\xi}\right)$ is trivial.

From Theorem 6.9 and Proposition 6.10 we recover a result proved in [13, 2.11]. Let $\varphi: \Gamma \rightarrow{ }^{L} W$ be an unramified Langlands parameter with $\varphi(\mathrm{F})=w \rtimes \vartheta$, where $w \in W$ and $\vartheta \in \operatorname{Aut}(R, B)$ is the automorphism induced by the action of $\mathrm{F}$ on 
the root datum of our quasi-split group $G$. The stable class $\mathcal{T}_{w}:=\mathcal{T}_{\varphi}$ consists of unramified maximal tori in $G$.

Corollary 6.11 ([13]). The rational classes of maximal tori in $\mathcal{T}_{w}$ are in bijection with the orbits of the centralizer $C_{W}(w \vartheta)$ on $\left[X_{w \vartheta}\right]_{\text {tor }}$ under the (linear) coinvariant representation. If $S_{\lambda} \in \mathcal{T}_{w}$ is a maximal torus in the rational class corresponding to $\lambda \in\left[X_{w \vartheta}\right]_{\text {tor }}$, then the stabilizer $C_{W}(w \vartheta, \lambda)$ is isomorphic to the small rational Weyl group of $S_{\lambda}$.

6.6.2. Tame parameters with odd inertial coinvariants. In this section we assume that $G$ is split over $k$. There is an extension

$$
1 \longrightarrow T[2] \longrightarrow \dot{W} \longrightarrow W \longrightarrow 1
$$

where $T[2]=\left\{t \in T: t^{2}=1\right\}$ and $\dot{W} \subset N(k)$ [38, Prop. 3]. We have $X / 2 X \simeq T[2]$, via evaluation at -1 , so that $T[2]$ is a linear representation of $W$ over $\mathbb{F}_{2}$.

Lemma 6.12. Assume that $w \in W$ is such that $\left.\operatorname{det}(1-w)\right|_{X}$ is odd. Then for any lift $\dot{w} \in \dot{W}$ of $w$, the projection $\dot{W} \rightarrow W$ induces an isomorphism $N_{\dot{W}}(\dot{w}) \stackrel{\simeq}{\longrightarrow}$ $N_{W}(w)$ between the normalizer of $\langle\dot{w}\rangle$ in $\dot{W}$ and the normalizer of $\langle w\rangle$ in $W$. This isomorphism restricts to an isomorphism on centralizers.

Proof. If $u \in N_{W}(w)$, then $u w u^{-1}=w^{q}$ for some $q$ relatively prime to the order of $w$. Choose an arbitrary lift $\dot{u} \in \dot{W}$, so that $\dot{u} \dot{w} \dot{u}^{-1}=\dot{w}^{q} t$, for some $t \in T[2]$. Since $w$ is conjugate to $w^{q}$, we have that $\operatorname{det}\left(1-w^{q}\right)=\operatorname{det}(1-w)$ is odd, so the map $1-w^{q}: T[2] \rightarrow T[2]$ is an isomorphism. If we choose $s \in T[2]$ so that $s^{1-w^{q}}=t$, then $(s \dot{u}) \dot{w}(s \dot{u})^{-1}=\dot{w}^{q}$. This proves that $N_{\dot{W}}(\dot{w}) \rightarrow N_{W}(w)$, as well as the restriction to centralizers, is surjective. To see injectivity, suppose $t \in T[2] \cap N_{\dot{W}}(\dot{w})$. Then for some integer $q$ we have $t \dot{w} t^{-1}=\dot{w}^{q}$. But $t \dot{w} t^{-1}=\dot{w} t^{w-1}$, so $t^{w-1}$ is a power of $\dot{w}$ and hence belongs to $T[2]^{w}$. Since $T[2]^{w}$ is trivial it follows that $t=1$.

Let $\mathcal{I} \triangleright \mathcal{I}^{+}$be the inertia and wild inertia subgroups of $\Gamma$. Suppose we have a parameter (=cocycle, since $G$ is split) $\varphi: \Gamma \rightarrow W$ which is trivial on $\mathcal{I}^{+}$.

The image $\varphi(\Gamma)$ is generated by two elements $w, u \in W$, where $w$ generates the inertial image $\varphi(\mathcal{I})$ and $u=\varphi(\mathrm{F})$ is the image of a Frobenius element $\mathrm{F} \in \Gamma$. Hence $u$ and $w$ must satisfy the relation $u w u^{-1}=u^{q}$ and $\varphi(\Gamma) \subset N(w)$. By Lemma 6.12. we may and shall choose our lift $\dot{\varphi}: \Gamma \rightarrow N(k)$ such that $\delta_{\dot{\varphi}}=1$.

The element $u$ normalizes on $C_{W}(w)$ and acts on $X_{w}$ via the coinvariant representation of $N_{W}(w)$. Recall that the latter action is via a symplectic similitude with multiplier $q$. The big rational Weyl group $W_{\varphi}(k)=C_{W}(w)^{u}$, the fixed points of $u$ in $C_{W}(w)$, and $H^{1}\left(k, T_{\varphi}\right)=\left(X_{w}\right)_{u}$ is the group of coinvariants of $u$ in $X_{w}$.

Corollary 6.13. Assume $G$ is split over $k$ and that the Langlands parameter $\varphi$ : $\Gamma \rightarrow W$ is tamely ramified, with inertial image generated by an element $w \in W$ having $\operatorname{det}(1-w)$ odd, and let $u=\varphi(\mathrm{F}) \in W$ be the image of a Frobenius element $\mathrm{F} \in \Gamma$. Then the rational classes of maximal tori in the stable class $\mathcal{T}_{\varphi}$ are in bijection with the orbits of $C_{W}(w)^{u}$ on $\left(X_{w}\right)_{u}$ under the coinvariant representation.

Remark. For $W$ of type $E_{8}$, we have $\operatorname{det}(1-w)$ is odd exactly when $w$ or $-w$ belongs to one of the conjugacy classes $E_{8}, A_{2} E_{6}\left(a_{2}\right)$ and $E_{8}\left(a_{i}\right)$ for $1 \leq i \leq 8$ (see Table 1). 
6.7. Tame totally ramified tori in split groups. Recall that $k$ is a finite extension of $\mathbb{Q}_{p}$, with residue cardinality $q$. In this section we generalize the computation of $\Delta_{\varphi}$ for $S L_{2}$ (see Proposition 6.7) to any $k$-split semisimple group $G$ with Weyl group $W$ and any tame and totally ramified parameter $\varphi: \Gamma \rightarrow W$ with elliptic regular image. This image $\varphi(\Gamma)=\varphi(\mathcal{I})$ is cyclic, generated by an elliptic regular element $w$ whose order $d$ is prime to $p$. Such parameters exist exactly when $q \equiv 1$ $\bmod d$. We will explicitly compute a cocycle $\delta_{w}: C_{W}(w) \rightarrow X_{w}$ in the class $\Delta_{\varphi}$ of Theorem 6.9. Thus, we reduce the classification of these tame totally ramified anisotropic tori in $G$ to the classification of orbits in $X_{w}$ under the coinvariant representation of $C(w)$, for all conjugacy classes of elliptic elements $w \in W$.

Let $E$ be the fixed field in $\bar{k}$ of $\operatorname{ker} \varphi$. Then $E / k$ is a totally ramified tame Galois extension of degree $d$ and $\varphi$ induces an isomorphism $\operatorname{Gal}(E / k) \simeq\langle w\rangle$. Let $\sigma \in \operatorname{Gal}(E / k)$ be the generator such that $\varphi(\sigma)=w$. Then $\sigma$ acts on the $E$-points $X \otimes E^{\times}$of the twisted torus $T_{w}:=T_{\varphi}$ via the automorphism $\sigma_{w}:=w \otimes \sigma$.

6.7.1. Explicit Tate-Nakayama duality. The first task is to give an explicit isomorphism

$$
X_{w} \stackrel{\sim}{\longrightarrow} H^{1}\left(k, T_{w}\right) .
$$

First, by the inflation-restriction sequence, we have

$$
H^{1}\left(k, T_{w}\right)=H^{1}\left(E / k, T_{w}(E)\right) .
$$

Since $\operatorname{Gal}(E / k)=\langle\sigma\rangle$, we have

$$
H^{1}\left(E / k, T_{w}(E)\right)=\frac{\left.\operatorname{ker} N_{\sigma_{w}}\right|_{T(E)}}{\left.\operatorname{im}\left(1-\sigma_{w}\right)\right|_{T(E)}}
$$

where

$$
N_{\sigma_{w}}=1+\sigma_{w}+\sigma_{w}^{2}+\cdots+\sigma_{w}^{d-1} \in \operatorname{End}(T(E))
$$

Since $E / k$ is Galois, we can choose a uniformizer $\varpi$ of $E$ such that $\varpi^{d} \in k$. We then have $\sigma(\varpi)=\zeta \varpi$, where $\zeta$ is a root of unity in $k$ of order $d$. Let $r=(q-1) / d$ and choose an $r$ th root $\sqrt[r]{\zeta}$ of $\zeta$ in the multiplicative group $\mathfrak{f}^{\times}$.

Lemma 6.14. The map $X \rightarrow T(E)$ given by evaluation at $\sqrt[r]{\zeta}$ induces an isomorphism

$$
X_{w} \stackrel{\sim}{\longrightarrow} H^{1}(E / k, T(E)) .
$$

Proof. Since $\operatorname{Gal}(E / k)$ acts trivially on $\mathfrak{f}$ and $w$ is elliptic, we have, for every $\mu \in X$,

$$
N_{\sigma_{w}}(\mu(\sqrt[r]{\zeta}))=\left(\left(1+w+w^{2}+\cdots+w^{d-1}\right) \mu\right)(\sqrt[n]{\zeta})=1
$$

hence $\mu(\sqrt[r]{\zeta})$ is a cocycle. If $\mu=(1-w) \eta$, for some $\eta \in X$, then $\mu(\sqrt[r]{\zeta})=$ $\eta(\sqrt[r]{\zeta}) \cdot \sigma_{w}[\eta(\sqrt[r]{\zeta})]^{-1}$ is a coboundary. Hence sending $\mu \mapsto \mu(\sqrt[r]{\zeta})$ gives a welldefined map

$$
X_{w} \longrightarrow H^{1}(E / k, T(E)) .
$$

Since both groups have the same order, it suffices to show that this map is injective. Suppose that

$$
\mu(\sqrt[r]{\zeta})=t^{-1} \cdot \sigma_{w}(t)
$$

for some $t \in T_{w}(E)$. Write

$$
E^{\times}=\langle\varpi\rangle \times \mathfrak{f}^{\times} \times\left(1+P_{E}\right)
$$


where $P_{E}$ is the prime ideal in the ring of integers of $E$. Since $T_{w}$ splits over $E$, we have

$$
T_{w}(E)=T(E)=X(\varpi) \times X\left(\mathfrak{f}^{\times}\right) \times X\left(1+P_{E}\right),
$$

where $X(*)=\{\mu(*): \mu \in X\}$. Accordingly, we write $t=\delta(\varpi) \cdot \eta(a) \cdot t_{+}$, with $\delta, \eta \in X, a \in \mathfrak{f}^{\times}$and $t_{+} \in X\left(1+P_{E}\right)$. Then we have

$$
\mu(\sqrt[r]{\zeta})=\frac{w \delta(\varpi)}{\delta(\varpi)} \cdot \frac{w \delta(\zeta) \cdot w \eta(a)}{\eta(a)} \cdot \frac{\sigma_{w}\left(t_{+}\right)}{t_{+}} .
$$

We compare factors on both sides: First, we must have $\sigma_{w}\left(t_{+}\right)=t_{+}$, so we may assume $t_{+}=1$. Second, we have $w \delta(\varpi) / \delta(\varpi)=1$, which implies that $(1-w) \delta=0$, since $\varpi$ has infinite order. Since $w$ is elliptic, this forces $\delta=0$. We therefore have $\mu(\sqrt[r]{\zeta})=w \eta(a) / \eta(a)$. Let $c$ be a generator of $\mathfrak{f}^{\times}$, and write $\sqrt[r]{\zeta}=c^{j}, a=c^{i}$, for some integers $i, j$. Since $j \mu$ and $i(w-1) \eta$ agree on $c$, we have

$$
j \mu-i(w-1) \eta \in(q-1) X \subset d X .
$$

$\operatorname{But} \operatorname{gcd}(j, d)=1$ since $\zeta$ has order $d$, so there is $\ell \in \mathbb{Z}$ such that

$$
\mu-\ell(w-1) \eta \in d X .
$$

From section 2.1 we have $d X \subset m X \subset(w-1) X$. It follows that $\mu \in(w-1) X$. Hence the map $X_{w} \rightarrow H^{1}(E / k, T(E))$ induced by evaluation at $\sqrt[r]{\zeta}$ is injective, as claimed.

6.7.2. Lifting Weyl group centralizers. Recall that $k$ is a finite extension of $\mathbb{Q}_{p}$, and that $K$ denotes the maximal unramified extension of $k$. Let $T(K)_{0}=X \otimes A_{K}^{\times}$, where $A_{K}$ is the ring of integers of $K$.

Lemma 6.15. Assume that $w \in W$ is elliptic, of order prime to $p$. Then the map $1-w: T(K)_{0} \rightarrow T(K)_{0}$ is surjective. That is, we have $H^{1}\left(\langle w\rangle, T(K)_{0}\right)=1$.

Proof. We give a constructive proof, using the polynomial

$$
\dot{M}(t)=\frac{m-M(t)}{1-t},
$$

where $M(t)$ is the minimal polynomial of $w$ and $m=M(1)$ (see section 2.1). Recall that

$$
(1-w) \dot{M}(w)=m \in \operatorname{End}(T)
$$

and $m$ divides the order of $w$. Hence $m$ is prime to $p$, and the $m$ th-power map is surjective on $A_{K}^{\times}$, hence on $T(K)_{0}$. Given $s \in T(K)_{0}$, choose $s_{1} \in T(K)_{0}$ such that $s_{1}^{m}=s$, and let $s_{2}=\dot{M}(w) s_{1}$. Then $s_{2} \cdot w\left(s_{2}\right)^{-1}=s_{1}^{m}=s$.

Let $\mathcal{A}(T, K)$ be the apartment of $T$ in Bruhat-Tits building of $G(K)$. Since $G$ is split over $K$, there exists a point $o \in \mathcal{A}(T, K)$, called special, such that the natural map $N \rightarrow W$ is surjective on the stabilizer $N(K)_{o}$ of $o$ in $N(K)$. That is, every element of $W$ has a lift in $N(K)_{o}$ and we have an exact sequence

$$
1 \longrightarrow T(K)_{0} \longrightarrow N(K)_{o} \longrightarrow W \longrightarrow 1
$$

Corollary 6.16. Assume that $w \in W$ is elliptic, of order prime to $p$, and let $\dot{w} \in N(K)_{o}$ be any lift of $w$. Then the natural map $N(K)_{o} \cap C_{N}(\dot{w}) \rightarrow C_{W}(w)$ is surjective. That is, every element of $C_{W}(w)$ has a lift in $N(K)_{o}$ commuting with the chosen lift $\dot{w}$ of $w$. 
Proof. The subgroup $\langle\dot{w}\rangle \subset N(K)_{o}$ generated by $\dot{w}$ acts on the exact sequence (38) by conjugation, and the action on the outer two terms factors through $\langle w\rangle$. The result follows from the vanishing of $H^{1}\left(\langle w\rangle, T(K)_{0}\right)$, proved in Lemma 6.15.

6.7.3. Lifting totally ramified parameters. Recall that $E / k$ be a totally ramified tame Galois extension of degree $d$, that $w \in W$ is an elliptic element of order $d$ and our parameter $\varphi: \Gamma \rightarrow W$ factors through an isomorphism $\operatorname{Gal}(E / k) \rightarrow\langle w\rangle$.

Assume also that $w$ lifts to an element of order $d$ in the adjoint group $\bar{G}$. This holds, for example, if $w$ is both elliptic and regular (cf. [31, 2.6]). The following is a refinement of Raghunathan's theorem in this situation.

Lemma 6.17. The isomorphism $\operatorname{Gal}(E / k) \stackrel{\sim}{\longrightarrow}\langle w\rangle$ lifts to a cocycle $\operatorname{Gal}(E / k) \rightarrow$ $N(E)$.

Proof. Since $w$ is elliptic, all lifts of $w$ in $N(\bar{k})$ are conjugate. Hence their $d$ th powers are conjugate and central, since $w$ has a lift of order $d$ in $\bar{G}$. It follows that there is an element $z$ in the center of $G$ which is the $d$ th power of any lift of $w$ in $G(\bar{k})$.

Choose a lift $\dot{w} \in N(k)$ of $w$. Let $\bar{T}$ be the image of $T$ in $\bar{G}$. Suppose we can find $t \in \bar{T}(E)$ such that $t^{-1} \sigma(t)$ has a lift $s \in T(\bar{k})$ which is conjugate to $\dot{w}$ in $G(\bar{k})$. Let $n=\operatorname{Ad}(t) \dot{w} \in N(E)$. Since $\dot{w} \in N(k)$, we have

$$
\sigma(n)=\operatorname{Ad}(\sigma(t)) \dot{w}=\operatorname{Ad}\left(t^{-1} \sigma(t)\right) n=s n s^{-1},
$$

so that

$$
n \cdot \sigma(n) \cdot \sigma^{2}(n) \cdots \sigma^{d-1}(n)=n \cdot\left(s n s^{-1}\right) \cdot\left(s^{2} n s^{-2}\right) \cdots\left(s^{d-1} n s^{1-d}\right)=(n s)^{d} s^{-d} .
$$

Since $s$ is conjugate to $\dot{w}$, its power $s^{d}$ is conjugate to $\dot{w}^{d}=z$, so $s^{d}=z ; n s$ is another lift of $w$ in $G(\bar{k})$, so $(n s)^{d}=z$. It follows that

$$
n \cdot \sigma(n) \cdot \sigma^{2}(n) \cdots \sigma^{d-1}(n)=z \cdot z^{-1}=1,
$$

so there is a unique cocycle $\operatorname{Gal}(E / k) \rightarrow N(E)$ sending $\sigma \mapsto n$.

It remains to produce an element $t \in \bar{T}(E)$ such that $t^{-1} \sigma(t)$ has a lift $s \in T(\bar{k})$ which is conjugate to $\dot{w}$ in $G(\bar{k})$. We work backwards. Since $\dot{w}$ is semisimple, it has a $G(\bar{k})$-conjugate $s \in T(\bar{k})$. The image $\bar{s}$ of $s$ in $\bar{G}$ is conjugate to a lift of $w$ in $\bar{G}$, hence $\bar{s}$ has order $d$. Since $\zeta$ generates the $d$ th roots of unity in $\bar{k}$, there is a coweight $\lambda \in X_{*}(\bar{T})$ such that $\bar{s}=\lambda(\zeta)$. Set $t=\lambda(\varpi) \in \bar{T}(E)$. Then

$$
\sigma(t)=\lambda(\sigma(\varpi))=\lambda(\zeta \varpi)=\bar{s} \cdot t .
$$

Hence $t^{-1} \sigma(t)=\bar{s}$ has the lift $s \in T(\bar{k})$ which is $G(\bar{k})$-conjugate to $\dot{w}$, as desired.

6.7.4. Computation of the cocycle. Assume that $w \in W$ is elliptic of order $d$, and that $w$ has a representative in the adjoint group $\bar{G}$ of the same order $d$, so that Corollary 6.16 applies and gives a lift $\dot{\varphi}: \Gamma \rightarrow N(E)$ of our parameter $\varphi: \Gamma \rightarrow$ $W$. We will compute the cocycle $\delta_{w}:=\delta_{\dot{\varphi}}: C(w) \rightarrow H^{1}\left(k, T_{w}\right)$ in terms of the isomorphism $X_{w} \rightarrow H^{1}\left(k, T_{w}\right)$ given in Lemma 6.14. Recall that $d \mid q-1$, since $E / k$ is Galois, and we set $r=(q-1) / d$.

From Lemma 6.17 and its proof, the parameter $\varphi: \Gamma \rightarrow W$ lifts to a cocycle $\dot{\varphi}: \Gamma \rightarrow N(E)$ factoring through $\operatorname{Gal}(E / k)$ and such that $\dot{\varphi}_{\sigma}=\operatorname{Ad}(t) \dot{w}$, where $t=\lambda(\varpi) \in \bar{T}(E)$ and $\lambda \in X_{*}(\bar{T})$ is a cocharacter such that the image of $\dot{w}$ in $\bar{G}$ is conjugate to $\lambda(\zeta)$. 
Proposition 6.18. The cocycle $\delta_{w}: C(w) \rightarrow X_{w}$ is given by

$$
\delta_{w}(x)=r(x-1) \lambda \bmod (1-w) X .
$$

Remark. Note that $\lambda \in \bar{X}=X_{*}(\bar{T})$, but $\lambda \notin X=X_{*}(T)$ in general. However, $(x-1) \lambda \in \mathbb{Z} \check{\Phi} \subset X$, so the formula for $\delta_{w}$ makes sense. If $w$ is elliptic and regular, one may take $\lambda$ to be the half-sum of the positive roots (cf. [31, 2.6] and example 6.7 .5 below).

Proof. The cocycle $\delta_{w}$ is determined by the values

$$
\delta_{w}(\dot{x})_{\sigma}=\dot{x} \dot{\varphi}_{\sigma} \sigma(\dot{x})^{-1} \dot{\varphi}_{\sigma}^{-1}
$$

where $\dot{x} \in N$ is a lift of $x \in C(w)$. By Corollary [6.16, we may and do choose $\dot{x} \in N(K)_{o}$ commuting with $\dot{w}$. We compute the class of $\delta_{w}(\dot{x})_{\sigma}$ in

$$
H^{1}\left(E / k, T_{w}(E)\right)=\frac{\left.\operatorname{ker} N_{\sigma_{w}}\right|_{T(E)}}{\left.\operatorname{im}\left(1-\sigma_{w}\right)\right|_{T(E)}},
$$

as follows.

$$
\delta_{w}(\dot{x})_{\sigma}=\dot{x} \varphi_{\sigma} \sigma(\dot{x})^{-1} \varphi_{\sigma}^{-1}=\dot{x} \varphi_{\sigma} \dot{x}^{-1} \varphi_{\sigma}^{-1}=\dot{x} \cdot \operatorname{Ad}(t) \dot{w} \cdot \dot{x}^{-1} \cdot \operatorname{Ad}(t) \dot{w}^{-1},
$$

since $\dot{x} \in N(K)=N^{\sigma}$. Let $\dot{t} \in T(\bar{k})$ be a lift of $t$. We rearrange the above product as

$$
\delta_{w}(\dot{x})_{\sigma}=\dot{x} \cdot \frac{\dot{t}}{w(\dot{t})} \cdot \dot{w} \dot{x}^{-1} \dot{w}^{-1} \cdot \frac{w(\dot{t})}{\dot{t}}=\dot{x} \cdot \frac{\dot{t}}{w(\dot{t})} \cdot \dot{x}^{-1} \cdot \frac{w(\dot{t})}{\dot{t}}=\frac{x(\dot{t})}{x w(\dot{t})} \cdot \frac{w(\dot{t})}{\dot{t}},
$$

since $\dot{x} \in C_{N}(\dot{w})$. We will modify this by a coboundary, after some preliminary remarks.

For any elements $h \in T(\bar{k})$ and $u, v \in W$, the quotient $u(h) / v(h)$ depends only on the image $\bar{h}$ of $h$ in $\bar{T}(\bar{k})$. It follows that if $\bar{h} \in \bar{T}(E)$, then $u(h) / v(h) \in T(E)$. If $\gamma \in \Gamma$ is a lift of $\sigma$, then $\gamma(\dot{t})$ and $\dot{t} s$ have the same image in $\bar{T}(E)$, namely $t \bar{s}$. It follows that

$$
\sigma\left(\frac{u(\dot{t})}{v(\dot{t})}\right)=\frac{u(\gamma(\dot{t}))}{v(\gamma(\dot{t}))}=\frac{u(\dot{t} s)}{v(\dot{t} s)} .
$$

Applying this to the element $b:=x(\dot{t}) / \dot{t}$, we have

$$
\frac{b}{\sigma_{w}(b)}=\frac{x(\dot{t})}{\dot{t}} \cdot \frac{w(\dot{t} s)}{w x(\dot{t} s)}=\frac{x(\dot{t})}{x w(\dot{t})} \cdot \frac{w(\dot{t})}{\dot{t}} \cdot \frac{w(s)}{x w(s)},
$$

since $x w=w x$. Comparing with equation (39), we find that

$$
\delta_{w}(\dot{x})_{\sigma} \equiv \frac{w(s)}{x w(s)} \bmod \operatorname{im}\left(1-\sigma_{w}\right)
$$

Now $\zeta=(\sqrt[r]{\zeta})^{r}$, so

$$
\frac{w(s)}{x w(s)}=\frac{w \lambda(\zeta)}{x w \lambda(\zeta)}=\frac{w \lambda(\sqrt[r]{\zeta})^{r}}{x w \lambda(\sqrt[r]{\zeta})^{r}}
$$

We find that the class of $\delta_{w}(\dot{x})$ in $H^{1}\left(E / k, T_{w}(E)\right)$ is that of $\mu(\sqrt[r]{\zeta})$, where, now in additive notation,

$$
\mu=r w(1-x) \lambda=r(1-x) \lambda \in X_{w},
$$

as claimed. 
Corollary 6.19. The class $\Delta_{\varphi}$ in $H^{1}\left(C(w), X_{w}\right)$ is trivial in any of the following cases:

1. $m \mid \frac{q-1}{d}$;

2. $\lambda \in X$;

3. $G$ is adjoint.

6.7.5. Example: The Coxeter element. Assume that $q=1+r h$, where $h$ is the Coxeter number of $G$ and $r$ is a positive integer, and let $w \in W$ be a Coxeter element. It is an old result of Kostant that $\check{\rho}(\zeta)$ is conjugate to a lift of $w$ in $\bar{G}$, where $\check{\rho}$ is half the sum of the positive coroots. Hence we may take $\lambda=\check{\rho}$ in Proposition 6.18. Since $C(w)=\langle w\rangle$, we write $\delta_{w}$ for the value $\delta_{w}(\dot{w})$. By Proposition 6.18, we have

$$
\delta_{w}=r(1-w) \check{\rho} \in X_{w} .
$$

Since $2 \check{\rho} \in X$, we have $2 \delta_{w}=0$. Since $(1-w) \check{\rho} \in(1-w) X$ iff $\check{\rho} \in X$, we find that $\delta_{w}=0$ iff either $r$ is even or $\check{\rho} \in X$. The simply-connected almost-simple groups with $\check{\rho} \notin X$ are

$$
S L_{2 n}, \quad S p_{2 n}, \quad E_{7}, \quad \operatorname{Spin}_{8 k+3}, \quad \operatorname{Spin}_{8 k+5}, \quad \operatorname{Spin}_{8 k+4}, \quad \operatorname{Spin}_{8 k+6} .
$$

If $r$ is odd, then $\delta_{w}=(w-1) \check{\rho} \in X_{w}$. Since $C_{W}(w)=\langle w\rangle$ acts trivially (via the linear action) on $X_{w}$, it follows that the rational classes in the stable class $\mathcal{T}_{w}$ are in bijection with the orbits of the involution $(w-1) \check{\rho} \in X_{w}$ acting by translation on $X_{w}$. For $S L_{2 n}$ this means there are $n$ rational classes in $\mathcal{T}_{w}$, in accordance with Propostion 6.7 for $S L_{2}$.

6.8. Supercuspidal $L$-packets. In this section we briefly indicate how coinvariant representations appear in the local Langlands correspondence. For more background on the local Langlands correspondence from this point of view, see [16], for example.

To simplify the discussion, we assume our $p$-adic group $G$ is simply-connected, semisimple and split over $k$. The dual group of $G$ the complex semisimple Lie group $\hat{G}$ of adjoint type whose root datum is dual to that of $G$. A supercuspidal parameter for $G$ is a continuous homomorphism $\varphi: \Gamma \rightarrow \hat{G}$ whose image has finite centralizer $A_{\varphi}=C_{\hat{G}}(\varphi)$. Two parameters are regarded as equivalent if they are conjugate by $\hat{G}$.

In this setting, the local Langlands conjecture predicts that each equivalence class of supercuspidal parameters $\varphi$ should correspond to a finite set $\Pi_{\varphi}$ of irreducible supercuspidal representations of $G(k)$ and that there should be a bijection between $\Pi_{\varphi}$ and the set of irreducible representations of the finite group $A_{\varphi}$.

Fix a supercuspidal parameter $\varphi: \Gamma \rightarrow \hat{G}$. The image $D=\varphi(\Gamma)$ is the Galois group of a finite extension $E / k$, with lower ramification filtration by normal subgroups of $D$ :

$$
D \geq D_{0} \geq D_{1} \geq \cdots \geq D_{m}>1,
$$

where $D_{0}$ is the inertia subgroup of $D$ and $D_{1}$, the wild ramification group, is the $p$-Sylow subgroup of $D_{0}$.

Suppose that for some $j \geq 0$ the group $D_{j}$ is contained in a maximal torus $\hat{T}$ of $\hat{G}$ and moreover that $D_{j}$ is in "general position" in $\hat{T}$, meaning that $\hat{T}=C_{\hat{G}}\left(D_{j}\right)$ 
is the full centralizer of $D_{j}$ in $\hat{G}$. Then $D$ is contained in the normalizer $\hat{N}$ of $\hat{T}$ and we have a cocycle

$$
\bar{\varphi}: \Gamma \stackrel{\varphi}{\longrightarrow} \hat{N} \longrightarrow W
$$

with coinvariant representation

$$
X_{\bar{\varphi}} \simeq \operatorname{Irr}\left(A_{\varphi}\right) \simeq H^{1}\left(k, T_{\bar{\varphi}}\right) .
$$

Thus, our $L$-packet $\Pi_{\varphi}$ should have the form

$$
\Pi_{\varphi}=\left\{\pi_{\lambda}: \lambda \in X_{\bar{\varphi}}\right\}
$$

and it is natural to expect the tori in the rational class containing $\lambda$ to be involved in the construction of the representation $\pi_{\lambda}$.

Such $L$-packets have been constructed in [13, 21, 30] and [17. We confine ourselves here to the settings of 13 and [21, where $\varphi$ is unramified. This means $j=0$ and the image of $D$ in $W$ is generated by an elliptic element $w \in W$ arising from the image $\varphi(\mathrm{F})$ of a Frobenius element in $\Gamma$.

Thus, $X_{\bar{\varphi}}=X_{w}$ is a coinvariant representation of $W_{\bar{\varphi}}(k)=C(w)$ as considered in the first part of the paper and the torus $T_{\bar{\varphi}}=T_{w}$ is unramified, so that the affine and linear actions of $C(w)$ on $X_{w}$ coincide, by Proposition 6.10.

The $L$-packet $\Pi_{\varphi}$ is constructed as follows. For each $\lambda \in X_{w}$ we have an embedding $\operatorname{Ad}(g): T_{w} \hookrightarrow T_{\lambda} \subset G$, where $g \in G(\bar{k})$ splits the class of $\lambda$ in $G$, and the image $T_{\lambda}$ is an anisotropic maximal torus of $G$ contained in a unique maximal compact subgroup $K_{\lambda}$. The parameter $\varphi$ determines a character $\chi: T_{w}(k) \rightarrow \mathbb{C}^{\times}$ by the local Langlands correspondence for $T_{w}$. Transporting $\chi$ to $T_{\lambda}(k)$ and using Deligne-Lusztig induction, we get a representation $\kappa_{\lambda}$ of $K_{\lambda}$, whence by compact induction an irreducible supercuspidal representation

$$
\pi(\chi, \lambda)=\operatorname{ind}_{K_{\lambda}}^{G(k)} \kappa_{\lambda} .
$$

These representations comprise the $L$-packet

$$
\Pi_{\varphi}=\left\{\pi(\chi, \lambda): \lambda \in X_{w}\right\} .
$$

To make this more explicit, we should give the conjugacy class of $K_{\lambda}$ in terms of $\lambda$. Since $T_{\lambda}$ determines $K_{\lambda}$, the latter depends only on the rational class of $T_{\lambda}$, which in turn depends only on the $C(w)$-orbit of $\lambda$. Thus, the $C(w)$-orbits in $X_{w}$ correspond to the maximal compact subgroups appearing as inducing data for the representations in $\Pi_{\varphi}$. More precisely, for $y \in C(w)$ we have the equivariance property 30

$$
\pi\left(\chi^{y}, \lambda\right) \simeq \pi\left(\chi, \varrho_{w}(y) \lambda\right)
$$

where $C(w)$ acts on characters of $T_{w}(k)$ via its isomorphism with the big rational Weyl group of $T_{w}$ from section 6.3. and $\varrho_{w}$ is the coinvariant representation of $C(w)$ on $X_{w}$.

The maximal compact subgroup $K_{\lambda}$ is the stabilizer in $G(k)$ of a vertex $x_{\lambda}$ in the Bruhat-Tits building $\mathcal{B}$ of $G(k)$. By conjugating, we can arrange that $x_{\lambda}$ belongs to the apartment $\mathcal{A}$ of $T$ in $\mathcal{B}$. The precise definition of $x_{\lambda}$ requires a choice of hyperspecial vertex $o \in \mathcal{A}$, by which we identify $\mathcal{A}$ with the vector space $\mathbb{R} \otimes X$. Modulo translations by $X$ (which do not change the $G(k)$-conjugacy class of $K_{\lambda}$ ), $x_{\lambda}$ is the image of $\lambda$ under the isomorphism

$$
(1-w)^{-1}: X_{w} \stackrel{\sim}{\longrightarrow}(1-w)^{-1} X / X \subset \mathcal{A} / X .
$$


The image of this map is the subgroup $\tilde{T}^{w}$ of elements fixed by $w$ in a maximal torus $\tilde{T}$ of the complex simply-connected group $\tilde{G}$ of the same type as $G$. The type of the vertex $x_{\lambda}$ (or $K_{\lambda}$ ) coincides with the type of the centralizer of the image of $x_{\lambda}$ in $\tilde{T}^{w}$. Therefore, the conjugacy class of $K_{\lambda}$ is the orbit type of $\lambda$, as discussed in section 2.3 for $E_{8}$. For $E_{8}$ we can read off the classes of maximal compact subgroups from Table 1. For example, if $w$ (the image of Frobenius under $\varphi$ ) belongs to the class $A_{1}^{2} A_{3}^{3}$ in $W$, then $\Pi_{\varphi}$ contains 64 representations induced from $K_{\lambda}$ 's of type $E_{8}(\times 1), D_{8}(\times 3), A_{1} E_{7}(\times 12)$ and $A_{3} D_{5}(\times 48)$.

\section{Appendix A. Further Remarks on ElLiptic trialities}

A.1. Elliptic trialities in $F_{4}$. Each case of elliptic trialities has special features, relating to other areas of mathematics. We explore these next, starting with the simplest nontrivial case.

The $F_{4}$ root lattice $X$ is the subgroup of $\mathbb{R}^{4}$ consisting of vectors whose coordinates are all integers or all half-integers. Identifying the standard basis of $\mathbb{R}^{4}$ with $1, i, j, k$, the Hamilton quaternion relations impart a ring structure to $X$. This ring $\mathcal{H}$, with underlying additive group $X$, is isomorphic to the endomorphism ring $\operatorname{End}(E)$ of the unique supersingular elliptic curve $E$ in characteristic two, with affine equation $y^{2}+y=x^{3}$. We refer to 34 for the basic facts about elliptic curves. The automorphism group of any elliptic curve has order dividing 24 [34, Thm. 10.1] and the curve $E$ attains this maximum: we have $\operatorname{Aut}(E)=\mathcal{H}^{\times} \simeq S L_{2}(3)$. This isomorphism is given by the action of $\operatorname{Aut}(E)$ on the group $E[3]=\{P \in E: 3 P=0\}$ of 3 -torsion points, on which the Weil pairing is a symplectic form invariant under $\operatorname{Aut}(E)$. The ring isomorphism $\theta: \mathcal{H} \rightarrow \operatorname{End}(E)$ intertwines the quadratic form $\langle x, x\rangle$ on $X$ with the form on $\operatorname{End}(E)$ given by the degree of an endomorphism. Hence $\theta$ sends the short roots in $X$ to the units $\operatorname{Aut}(E)$. The Frobenius endomorphism $\mathrm{F}$ of $E$ has degree two, so $\theta$ sends the long roots in $X$ to the twisted Frobenius endomorphisms $\sigma \mathrm{F}$ with $\sigma \in \operatorname{Aut}(E)$.

Fix an elliptic triality $w \in W\left(F_{4}\right)$. Proposition 4.2 shows that

$$
C_{W\left(D_{4}\right)}(w) \simeq S L_{2}(3)
$$

The element $\omega:=\theta(w \cdot 1) \in \operatorname{Aut}(E)$ satisfies

$$
\theta(w \lambda)=\theta(\lambda) \omega, \quad \text { for all } \lambda \in \mathcal{H} .
$$

Since $\omega$ has order three, it fixes a unique line in the two-dimensional $\mathbb{F}_{3}$-vector space $E[3]=\{P \in E: 3 P=0\}$. Let $P$ be a nonidentity point in this line. Then the map

$$
\mathcal{H} \longrightarrow E[3], \quad A \mapsto \theta(A) \cdot P
$$

induces an an $S L_{2}(3)$-equivariant isomorphism

$$
X_{w}=\mathcal{H} /(1-w) \mathcal{H} \stackrel{\sim}{\longrightarrow} E[3] .
$$

A.2. Elliptic trialities in $E_{6}$. Let us change coordinates slightly, and view the elliptic curve $E$ above as defined in $\mathbb{P}^{2}$ by the cubic polynomial $f=X^{2} Z+Y^{3}+X Z^{2}$. The 3 -torsion points on any elliptic curve are also the inflection points, hence are independent of the choice of origin defining the group structure. For our curve $E$, the 3 -torsion points coincide with the $\mathbb{F}_{4}$-rational points:

$$
E[3]=E\left(\mathbb{F}_{4}\right) .
$$


The polynomial $f$ may be viewed as a hermitian form on $\mathbb{F}_{4}^{3}$, and $E\left(\mathbb{F}_{4}\right)$ is the set of $f$-isotropic lines in $\mathbb{F}_{4}^{3}$. The projective unitary group $P U_{3}(2)$ of $f$, of order $9 \cdot 24$, acts transitively on the curve $E$ with group structure ignored. The stabilizer of a point in $E\left(\mathbb{F}_{4}\right)$ is a Borel subgroup in $P U_{3}(2)$ and is isomorphic to $S L_{2}(3)$. Thus we may identify the points in $E\left(\mathbb{F}_{4}\right)$ with the Borel subgroups of $P U_{3}(2)$. Given a Borel subgroup $B$, and letting $E_{B}$ be the elliptic curve (over $\mathbb{F}_{4}$ ) defined by $f$ with identity element $B$, we have $\operatorname{Aut}\left(E_{B}\right)=B$. To see this explicitly, let $B$ be the stabilizer of $O=[1,0,0] \in E$. Then ([34, p. 327]) $B=\operatorname{Aut}(E)$ is given in $X, Y, Z$ coordinates by the projective matrices:

$$
\left[\begin{array}{ccc}
1 & u s & t \\
0 & u & s^{2} \\
0 & 0 & 1
\end{array}\right], \quad u \in \mathbb{F}_{4}^{\times}, \quad[s, t, 1] \in E\left(\mathbb{F}_{4}\right) .
$$

The subgroup with $u=1$ is the quaternion group $Q_{8}$; these eight automorphisms happen to be parametrized by the points in $E\left(\mathbb{F}_{4}\right)$ distinct from $O$. This is explained by the Bruhat decomposition: since $\mathrm{PU}_{3}(2)$ has rank one, the 2-Sylow subgroup of any Borel subgroup acts simply-transitively, by conjugation, on the remaining Borel subgroups.

The group $(E, O)$ acts on itself by translations, and this action turns out to be linear on $E\left(\mathbb{F}_{4}\right)$. To see this, it suffices, by the transitivity of $Q_{8}$, to note that translation by the point $P=[0,0,1]$ is given by the linear map $[X, Y, Z] \mapsto$ $[Z, Y, X+Z]$. Thus, $E\left(\mathbb{F}_{4}\right)$ embeds in $P U_{3}(4)$ as a normal subgroup, and we have

$$
P U_{3}(2)=E\left(\mathbb{F}_{4}\right) \rtimes S L_{2}(3) .
$$

An elliptic triality $w \in W\left(F_{4}\right)$ is also an elliptic triality in $W\left(E_{6}\right)$. Let $\zeta \in \overline{\mathbb{Q}}^{\times}$ have order three, let $X$ be the root lattice of $E_{6}$ and let $V_{K}$ be the $K$-vector space $V=\mathbb{Q} \otimes X$, where $\zeta$ acts on $V$ via $w$. The group $C_{W\left(E_{6}\right)}(w)$ preserves the hermitian form $h$ on $V_{K}$ (see section 3.2). Let $X_{K}$ be the abelian group $X$, viewed as a $\mathbb{Z}[\zeta]$ module. Since 2 remains prime in $\mathbb{Z}[\zeta]$, the form $h$ induces a hermitian form on the vector space $X_{K} / 2 X_{K} \simeq \mathbb{F}_{4}^{3}$. This gives an isomorphism

$$
C_{W\left(E_{6}\right)}(w) \simeq U_{3}(2)
$$

in which $w$ maps to a scalar matrix in $U_{3}(2)$, so that

$$
C_{W\left(E_{6}\right)}(w) /\langle w\rangle \simeq P U_{3}(2) .
$$

Since all hermitian forms in three variables are equivalent, we see that $C_{E_{6}}(w) /\langle w\rangle$ is the automorphism group of the curve $E$ with group structure ignored.

There is a connection with Weil representations. In section 4.1, we have seen that $C_{A\left(E_{6}\right)}(w)$ is a maximal parabolic subgroup in $S p_{4}(3)$ with Heisenberg group $H$ for unipotent radical. The Levi subgroup of $C_{A\left(E_{6}\right)}(w)$ is $\mathbb{F}_{3}^{\times} \times S L_{2}(3)$, where the first factor is generated by the graph automorphism of $E_{6}$. It follows that

$$
C_{W\left(E_{6}\right)}(w)=S L_{2}(3) \ltimes H .
$$

The center of $H$ is generated by $w$. From section 3.1, the eigenspaces $\bar{V}(w, \zeta)$ and $\bar{V}\left(w, \zeta^{2}\right)$ are three-dimensional irreducible representations of $C_{W\left(E_{6}\right)}(w)$, affording the central characters $w \mapsto \zeta, w \mapsto \zeta^{2}$ of $H$. It follows that $\bar{V}(w, \zeta)$ and $\bar{V}\left(w, \zeta^{2}\right)$ are the Weil representations of $C_{W\left(E_{6}\right)}(w)=S L_{2}(3) \ltimes H$ [15, 2.4].

The action of $C_{W\left(E_{6}\right)}(w)$ on $X_{K}$ and $X_{w}$ may also be seen in the Hessian configuration, much studied in the nineteenth century, for which a complete account in the classical style can be found in [4, 7.3]. The projective curve $C_{\lambda}$ with equation 
$x^{3}+y^{3}+z^{3}-\lambda x y z=0$ is singular precisely for $\lambda=\infty, 1, \zeta, \zeta^{2}$, where $C_{\lambda}$ becomes a triangle. The resulting twelve lines in $\mathbb{P}^{2}$ form the Hessian configuration, whose group of collineations is $P U_{3}(2)$. This is proved in [4] by a judicious labelling of coordinates, which identifies the Hessian configuration with the configuration of all lines in the affine plane over $\mathbb{F}_{3}$, whose symmetry group is $P U_{3}(2)$, as we have seen in (43).

This can be seen more naturally using the coinvariants $X_{w}$ and the twelve $K$ equivalence classes of roots in $E_{6}$ as follows. Each $K$-equivalence class $S$ determines a line $K S$ in $V_{K}$, whence a hyperplane in the complexified dual space $\mathbb{V}=\left[V_{K} \otimes_{K}\right.$ $\mathbb{C}]^{*}$. These hyperplanes give the Hessian configuration in $\mathbb{P}(\mathbb{V})$. On the other hand, by Lemma 4.4, $S$ determines a nonsingular line in $X_{w} \simeq \mathbb{F}_{3}^{3}$, whence a hyperplane in the dual space $X_{w}^{*}$. All hyperplanes in $X_{w}^{*}$ arise except the one annihilated by the singular line in $X_{w}$, which is the radical of the form $\langle,\rangle_{w}$. We denote this singular hyperplane by $H_{0}$. Thus, the twelve $K$-equivalence classes $S$ are in bijection with the lines in the affine space $\mathbb{P}\left(X_{w}^{*}\right)-\left\{H_{0}\right\}$. Since each of these modular lines comes from reduction modulo $P=(1-\zeta) \mathbb{Z}[\zeta]$ of a complex line in $\mathbb{P}(\mathbb{V})$, this shows that the complex and modular Hessian configurations coincide.

A.3. Elliptic trialities in $E_{8}$. Let $w$ be an elliptic triality in $W\left(E_{8}\right)$, let $X$ be the $E_{8}$ root lattice, let $V=\mathbb{Q} \otimes X$, and let $K=\mathbb{Q}(\zeta)$ be generated by a root of unity of order three. Each $K$-equivalence class of roots is an orbit of $-w$, and is the vertex set of one of Coxeter's 40 planar hexagons (cf. [10, p. 480]). The centralizer $C(w)$ of $w$ in $W\left(E_{8}\right)$ has order

$$
|C(w)|=12 \cdot 18 \cdot 24 \cdot 30=155520 .
$$

Here $12,18,24,30$ are the degrees of $W\left(E_{8}\right)$ which are divisible by 3 . We have

$$
C(w)=\langle w\rangle \times C(w)^{\prime},
$$

where $C(w)^{\prime}$ is the subgroup of $C(w)$ having determinant one on $V_{K}$ and has order

$$
\left|C(w)^{\prime}\right|=51840 .
$$

From Proposition 4.2, the representation of $C(w)^{\prime}$ on $X_{w}$ gives an isomorphism

$$
C(w)^{\prime} \simeq S p_{4}(3) .
$$

Just as for $E_{6}$, there is also a mod 2 picture. Again the hermitian form $h$ on $V_{K}$ becomes a cubic polynomial $h_{2}$ on $X_{K} / 2 X_{K}$. This time we get a two-fold covering

$$
1 \longrightarrow\{ \pm 1\} \longrightarrow C(w) \longrightarrow U_{4}(2) \longrightarrow 1
$$

under which $w$ maps to a generator of the center of $U_{4}(2)$. This last group has order

$$
\left|U_{4}(2)\right|=2^{6}\left(2^{4}-1\right)\left(2^{3}+1\right)\left(2^{2}-1\right)(2+1)=2^{6} \cdot 3^{5} \cdot 5
$$

and preserves the nonsingular cubic surface $S \subset \mathbb{P}^{3}$ defined by $h_{2}$.

A line on $S\left(\mathbb{F}_{4}\right)$ is an $h$-isotropic plane in $\mathbb{F}_{4}^{4}$. The group $U_{4}(2)$ acts transitively on isotropic planes and the stabilizer of one such is a semidirect product $G L_{2}(4) \ltimes \mathbb{F}_{2}^{4}$, of order $2^{6}\left(2^{4}-1\right)\left(2^{2}-1\right)$. Since

$$
\frac{2^{6}\left(2^{4}-1\right)\left(2^{3}+1\right)\left(2^{2}-1\right)(2+1)}{2^{6}\left(2^{4}-1\right)\left(2^{2}-1\right)}=27
$$


this shows that $U_{4}(2)$ acts transitively on the lines in $S$ and that every such line is rational over $\mathbb{F}_{4}$. Not all lines are rational over $\mathbb{F}_{2}$, so the action of $\operatorname{Gal}\left(\mathbb{F}_{4} / \mathbb{F}_{2}\right)$ on the set of lines is nontrivial.

The symmetry group of the configuration of 27 lines in $S$ is $W\left(E_{6}\right)$, whose order $\left|W\left(E_{6}\right)\right|=2^{7} \cdot 3^{4} \cdot 5$ is twice that of $P U_{4}(2)$. Since $W\left(E_{6}\right)$ has a unique character of order two, namely the sign character $\epsilon$, the action of $U_{4}(2)$ on the configuration of lines in $S$ gives an isomorphism of simple groups

$$
P U_{4}(2) \stackrel{\sim}{\longrightarrow} W\left(E_{6}\right)_{+}=\operatorname{ker} \epsilon .
$$

The nonidentity coset of $P U_{4}(2)$ in $W\left(E_{6}\right)$ contains the nontrivial element of $\operatorname{Gal}\left(\mathbb{F}_{4} / \mathbb{F}_{2}\right)$ acting on the lines in $S$. Lifting back to the two-fold cover $C(w)$ of $U_{4}(2)$ via (45), we find that

$$
C(w) \simeq\langle w\rangle \times \widetilde{W\left(E_{6}\right)_{+}},
$$

where $\widetilde{W\left(E_{6}\right)_{+}}$is a two-fold cover of $W\left(E_{6}\right)_{+}$. Comparing with (44), we recover the isomorphism (cf. 7])

$$
S p_{4}(3) \simeq \widetilde{W\left(E_{6}\right)_{+}} .
$$

Since $S p_{4}(3)$ equals its own derived group, the covering

$$
\begin{array}{cccc}
S p_{4}(3) & \hookrightarrow & S U_{4} \\
\downarrow & & \downarrow \\
W\left(E_{6}\right)_{+} & \hookrightarrow & S O_{6}
\end{array}
$$

is nonsplit, in complete analogy with the binary tetrahedral covering

$$
\begin{array}{cccc}
\mathrm{Sp}_{2}(3) & \hookrightarrow & \mathrm{SU}_{2} \\
\downarrow & & \downarrow \\
W\left(\mathrm{~A}_{3}\right)_{+} & \hookrightarrow & \mathrm{SO}_{3} .
\end{array}
$$

In summary, we have three avatars of the group $C(w)^{\prime}$ of order 51840:

$$
\begin{aligned}
C(w)^{\prime} & \simeq S p_{4}(3) \quad \text { from the coinvariant representation on } X_{w}, \\
& \simeq P U_{4}(2) \quad \text { from the hermitian form } h_{2} \text { on } X_{K} / 2 X_{K}, \\
& \simeq \widetilde{W\left(E_{6}\right)_{+}} \quad \text { from the } 27 \text { lines on the cubic surface } S:\left(h_{2}=0\right) .
\end{aligned}
$$

The eigenspaces $\bar{V}(w, \zeta)$ and $\bar{V}\left(w, \zeta^{2}\right)$ are in duality via the pairing $\langle$,$\rangle on \bar{V}$ and afford the two distinct four-dimensional representations of $S p_{4}(3)$ over $\overline{\mathbb{Q}}[7$. The exterior squares of these representations are irreducible and isomorphic to one another; let

$$
\Lambda:=\Lambda^{2} \bar{V}(w, \zeta) \simeq \Lambda^{2} \bar{V}\left(w, \zeta^{2}\right)
$$

As a representation of $S p_{4}(3), \Lambda$ is the unique cuspidal unipotent representation, denoted by $\theta_{10}$ in [37. As a representation of $U_{4}(2), \Lambda$ is the unipotent representation corresponding to the partition $4=2+1+1$. As shown in [19], the representation $\Lambda \otimes \overline{\mathbb{Q}}_{\ell}$ can be realized on the quotient of the $\ell$-adic cohomology group $H^{2}(S)$ by the one-dimensional subspace spanned by a hyperplane section. We note that, for the elliptic trialities in $F_{4}$ and $E_{8}$, the middle exterior powers of $\bar{V}(w, \zeta)$ are realized in the cohomology groups $H^{1}(E)$ and $H^{2}(S)$, respectively. 
A.4. Maschke's view. The representation of $C(w)^{\prime}$ on $V_{K}$ gives a subgroup $G \subset$ $G L_{4}(K)$ of order 51840 which was discovered by Witting and whose invariant theory was determined by Maschke [25] in 1889, before the theory of root systems was developed. Maschke first shows that $G$ is a nonsplit two-fold cover of its image $G /\{ \pm 1\} \subset P G L_{4}(K)$. This is the covering (45) above. Next, Maschke computes the stabilizer $H$ in $G$ of a coordinate hyperplane $V_{0} \subset V_{K}$ and shows that the image $H^{\prime}$ of $H$ in $P G L\left(V_{0}\right)$ is the Hessian group of order 216, concluding from this that $|H|=6 \cdot 216$. In our context, $H$ is the image of $C_{E_{7}}(w)=\{ \pm 1\} \times C_{E_{6}}(w)$ in $C(w)^{\prime}$ and the projection $C_{E_{6}}(w) \rightarrow H^{\prime}$ is the Hessian covering from section $\AA .2$ (note that the present $w$ contains an elliptic triality in $W\left(E_{6}\right)$ as a factor).

Maschke goes on to show that the $H$ - and $G$-invariant polynomials on $V_{0}$ and $V_{K}$ are generated by explicit polynomials whose degrees $(6,12,18$ and $12,18,24,30$, respectively) are as we would find today from Springer's theory.

\section{Appendix B. Embedding $W\left(H_{4}\right)$ in $W\left(E_{8}\right)$}

Let $w \in W\left(E_{8}\right)$ be cyclotomic of order 10. The operator $\tau=w+w^{-1} \in \operatorname{End}(V)$ satisfies the equation $\tau^{2}=\tau+1$ of the golden ratio. The field $k=\mathbb{Q}(\sqrt{5}) \simeq \mathbb{Q}(\tau)$ embeds in $\operatorname{End}(V)$, via $\frac{1}{2}(1+\sqrt{5}) \mapsto \tau$, and we let $V_{k}$ be the $k$-vector space with underlying abelian group $V$. We will show that the subgroup $C(\tau)$ of elements in $W\left(E_{8}\right)$ commuting with $\tau$ in $\operatorname{End}(V)$ is a reflection group on $V_{k}$, isomorphic to the noncrystallographic Coxeter group $W\left(H_{4}\right)$. From the equation

$$
w^{2}-w+1-w^{-1}+w^{-2}=0
$$

it follows that

$$
\langle\alpha, w \alpha\rangle=\left\langle\alpha, w^{2} \alpha\right\rangle+1
$$

for every $\alpha \in R$, which implies that $\langle\alpha, w \alpha\rangle \in\{0,1\}$. For $i \in\{0,1\}$, let $R_{i}=\{\alpha \in$ $R:\langle\alpha, w \alpha\rangle=i\}$.

Lemma B.1. The operator $\tau$ maps $R_{0}$ bijectively onto $R_{1}$, and has the following properties:

1. $\langle\alpha, \tau \alpha\rangle=0$;

2. $s_{\alpha} s_{\tau \alpha} \in C(\tau)$ for all $\alpha \in R_{0}$;

3. the $w$-orbits of $\alpha$ and $\tau \alpha$ comprise a root subsystem of type $A_{4}$.

Proof. If $\langle\alpha, w \alpha\rangle=0$, then $\left\langle\alpha, w^{2} \alpha\right\rangle=-1$ by (46), so that $\alpha+w^{2} \alpha \in R$. Hence $\tau \alpha=w^{-1}\left(\alpha+w^{2} \alpha\right) \in R$. It is straightforward to check that

$$
\langle\tau \alpha, \tau w \alpha\rangle=1 \quad \text { and } \quad\langle\alpha, \tau \alpha\rangle=0 .
$$

The first of these equations shows that $\tau \alpha \in R_{1}$. Using $\tau^{2}=\tau+1$ a straightforward calculation shows that $\tau$ commutes with $s_{\alpha} s_{\tau \alpha}$, proving 2 . For the bijectivity, note that $\tau-1$ sends $R_{1} \rightarrow R_{0}$ and $\tau(\tau-1)=1$. For 3 , one checks that $\left\{w \alpha, w^{3} \alpha, \alpha, w^{2} \alpha\right\}$ forms a base of an $A_{4}$.

From Lemma B.1, it follows that the $k$-equivalence classes are the subsystems of $R$ of the form

$$
S=\{ \pm \alpha, \pm \tau \alpha\} \simeq 2 A_{1}, \quad \text { for } \quad \alpha \in R_{0} .
$$

These give $60 k$-reflections $s_{\alpha} s_{\tau \alpha}$ generating a reflection subgroup $C(\tau)^{\circ} \subset C(\tau)$. From the classification of real reflection groups, we see that $C(\tau)^{\circ}$ is the Coxeter group of type $H_{4}$. We no longer know a priori that $C(\tau)$ is a reflection group. 
To see the Coxeter generators of $C(\tau)^{\circ}$, number the simple roots of $E_{8}$ as shown:

$$
\begin{array}{lllllll}
1 & 2 & 3 & 4 & 5 & 6 & 7 \\
& & & 8 & &
\end{array}
$$

and let $s_{i}$ be the corresponding simple reflections. Choose a "bipartite" Coxeter element

$$
v=s_{2} s_{4} s_{6} s_{8} s_{1} s_{3} s_{5} s_{7}
$$

(writing $s_{i}$ for $s_{\alpha_{i}}$ ). The element $w=v^{3}$ is cyclotomic of order ten. One checks that

and that

$$
\alpha_{1}, \alpha_{2}, \alpha_{3}, \alpha_{8} \in R_{0}, \quad \alpha_{4}, \alpha_{5}, \alpha_{6}, \alpha_{7} \in R_{1}
$$

$$
\tau: \alpha_{1} \mapsto \alpha_{7}, \quad \alpha_{2} \mapsto \alpha_{6}, \quad \alpha_{3} \mapsto \alpha_{5}, \quad \alpha_{8} \mapsto \alpha_{4} .
$$

Thus we recover the "inflation map" of [26] (defined there in terms of icosians). As in [ibid.] the Coxeter relations in $W\left(E_{8}\right)$ immediately imply that the $k$-reflections

$$
r_{1}=s_{1} s_{7}, \quad r_{2}=s_{2} s_{6}, \quad r_{3}=s_{3} s_{5}, \quad r_{4}=s_{4} s_{8}
$$

satisfy the Coxeter relations of $W\left(H_{4}\right)$, according to the diagram $1-2-3-{ }^{5} 4$.

We now show that $C(\tau)^{\circ}=C(\tau)$. Since its minimal polynomial is irreducible mod 2, $\tau$ gives an $\mathbb{F}_{4}$-structure to the abelian group $X / 2 X$. The quotient $C(\tau) / \pm 1$ is the subgroup of $O_{8}^{+}(2)$ acting $\mathbb{F}_{4}$-linearly on $X / 2 X$. Hence $C(\tau) / \pm 1$ is an orthogonal group in four variables over $\mathbb{F}_{4}$. There are two such groups: $O_{4}^{\epsilon}(4)$, where $\epsilon= \pm$. Since $\left|O_{4}^{\epsilon}(4)\right|=2 \cdot 4^{2}\left(4^{2}-\epsilon 1\right)\left(4^{2}-1\right)$ and $C(\tau)$ contains the subgroup $C(\tau)^{\circ}=W\left(H_{4}\right)$ of order $120^{2}$, it follows that $\epsilon=+$ and that $C(\tau)^{\circ}=C(\tau)$ as claimed.

\section{ACKNOWLEDGMENTS}

I thank D. Vogan for his comments on an earlier version of this paper, S. DeBacker for pointing me to Raghunathan's article [27, and B. Gross for filling the margins with meticulous criticism. Some of the results on tori in section 6 were motivated by recent work with Gross and J.-K. Yu.

\section{REFERENCES}

[1] J. D. Adler, Refined anisotropic K-types and supercuspidal representations, Pacific J. Math., 185 (1998), pp. 1-32. MR1653184 (2000f:22019)

[2] E. Bayer-Fluckiger and I. Suarez, Ideal lattices over totally real number fields and Euclidean minima, Arch. Math. (Basel), 86 (2006), pp. 217-225. MR2215310(2007d:11072)

[3] N. Bourbaki, Lie Groups and Lie Algebras, Chaps. 4-6, Springer-Verlag, Berlin, 2002. MR1890629 (2003a:17001)

[4] E. Brieskorn and H. Knörrer, Plane Algebraic Curves, Birkhäuser, 1981. MR646612 (83i:14001)

[5] R. Carter, Finite Groups of Lie Type, Wiley, 1985. MR794307 (87d:20060)

[6] _ Conjugacy classes in the Weyl group, Compositio Math., 25, (1972), pp. 1-59. MR0318337 (47:6884)

[7] J.H. Conway et al., ATLAS of finite groups, Clarendon Press, Oxford, 1985. MR827219 (88g:20025)

[8] J.H. Conway and N.J.A. Sloan, Sphere Packings, Lattices and Groups, Springer, 1999. MR.1662447 (2000b:11077)

[9] J.H. Conway and D.A. Smith, On quaternions and octonions, A.K.Peters, 2003. MR1957212 (2004a:17002)

[10] H.S.M. Coxeter, The polytope $2_{21}$, whose twenty-seven vertices correspond to the lines on the general cubic surface, Am. J. Math., 61 (1940), pp. 457-486. MR0002180(2:10a) 
[11] _ Regular polytopes, Dover, 1973. MR0370327 (51:6554)

[12] S. DeBacker, Parametrizing conjugacy classes of maximal unramified tori, Mich. Math. J., 54 (2006), pp. 157-178. MR2214792(2007d:22012)

[13] S. DeBacker and M. Reeder, Depth-zero supercuspidal L-packets and their stability, Annals of Math., 169, no. 3 (2009), pp. 795-901. MR.2480618 (2010d:22023)

[14] P. Deligne and G. Lusztig, Representations of reductive groups over finite fields, Ann. of Math., 103 (1976), pp. 103-161. MR0393266 (52:14076)

[15] P. Gerardin, Weil representations associated to finite fields, Jour. of Alg., 46 (1977), pp. 54101. MR0460477 (57:470)

[16] B. H. Gross and M. Reeder, From Laplace to Langlands via representations of orthogonal groups, Bull. Amer. Math. Soc., 43 (2006), pp. 163-205. MR2216109 (2007a:11159)

[17] _ Arithmetic invariants of discrete Langlands parameters, Duke Math. J. 154 (2010), no. 3, 431-508. MR2730575

[18] B. H. Gross, M. Reeder, J.-K. Yu, work in progress

[19] R. Hotta and K. Matsui On a Lemma of Tate-Thompson, Hiroshima Math. J., 8 (1978), pp. 255-268. MR0486178 (58:5958)

[20] V. Kac Infinite dimensional Lie algebras, third ed., Cambridge, 1995.

[21] D. Kazhdan and Y. Varshavsky, Endoscopic decomposition of characters of certain cuspidal representations, Electron. Res. Announc. Amer. Math. Soc., 10 (2004), pp. 11-20. MR2048427 (2006d:22024)

[22] R. Kottwitz, Stable trace formula: cuspidal tempered terms, Duke Math. J., 51, (1984) pp. 611-650. MR757954 (85m:11080)

[23] _ Stable trace formula: elliptic singular terms, Math. Ann., 275 (1986), pp. 365-399. MR858284 (88d:22027)

[24] Isocrystals with additional structure. II, Compositio Math., 109 (1997), pp. 255-339. MR.1485921 (99e:20061)

[25] H. Maschke, Aufstellung des vollen Formensystems einer quaternären Gruppe von 51840 linearen Substitutionen, Math. Ann., 33 (1889), pp. 317-344. MR1510546

[26] R. Moody and J. Patera, Quasicrystals and icosians, J. Phys. A: Math. Gen., 26 (1993), pp. 2829-2853. MR1236147 (94f:52030)

[27] M.S. Raghunathan, Tori in quasi-split groups, J. Ramanujan Math. Soc., 19 (2004), no. 4, pp. 281-287. MR2125504 (2005m:20114)

[28] M. Rapaport A guide to the reduction modulo $p$ of Shimura varieties, Astérisque, 298 (2005), pp. 271-318. MR 2141705 (2006c:11071)

[29] M. Reeder, Level-two structure of simply-laced Coxeter groups, J. Alg, 285 (2005), pp. 29-57. MR2119103 (2005k:20096)

[30] _ Supercuspidal L-packets of positive depth and twisted Coxeter elements, J. Reine Angew. Math., 620, (2008), pp. 1-33. MR2427973 (2009e:22019)

[31] _ Torsion automorphisms of simple Lie algebras, L'Ens. Math., to appear.

[32] J.-P. Serre, Galois Cohomology, Springer-Verlag, 2002. MR1867431 (2002i:12004)

[33] G.C. Shephard, J.A. Todd, Finite unitary reflection groups, Canadian J. Math., 6 (1954), pp. 274-304. MR0059914 (15:600b)

[34] J. Silverman, The Arithmetic of Elliptic Curves, Springer-Verlag, 1986. MR817210 (87g:11070)

[35] T. Springer, Regular elements in finite reflection groups, Inv. Math., 25 (1974), pp. 159-198. MR0354894(50:7371)

[36] T. A. Springer and R. Steinberg, Conjugacy classes, Seminar in algebraic groups and related finite groups, Lecture Notes in Math., 131 (1970), pp. 167-266. MR0268192 (42:3091)

[37] B. Srinivasan, Characters of the finite symplectic group $S p(4, q)$, Trans. Amer. Math. Soc., 131 (1968), pp. 488-525. MR0220845 (36:3897)

[38] J. Tits, Sur les constantes de structure et le théorèm d'existence des algèbres de Lie semisimples, Inst. Hautes Études Sci. Publ. Math., No. 31 (1966), pp. 21-58. MR0214638(35:5487)

[39] E.B. Vinberg, The Weyl group of a graded Lie algebra, Math. USSR Isvestiya, 10 (1976), no. 3, pp. 463-495. MR0430168 (55:3175)

Department of Mathematics, Boston College, Chestnut Hill, Massachusetts 02467 E-mail address: reederma@bc.edu 